A post print of a published manuscript at Computers and Geosciences \title{
correction and noise reduction
}

\author{
Zhang Yunjun ${ }^{\mathrm{a}, *}$, Heresh Fattahi ${ }^{\mathrm{b}}$, Falk Amelung ${ }^{\mathrm{a}}$
}

${ }^{a}$ Rosenstiel School of Marine and Atmospheric Science, University of Miami, Miami, Florida, USA

b Jet Propulsion Laboratory, California Institute of Technology, Pasadena, California, USA

*Correspondence to Z. Yunjun, yzhang@rsmas.miami.edu

\section{Abstract}

We present a review of small baseline interferometric synthetic aperture radar (InSAR) time series analysis with a new processing workflow and software implemented in Python, named MintPy (https://github.com/insarlab/MintPy). The time series analysis is formulated as a weighted least squares inversion. The inversion is unbiased for a fully connected network of interferograms without multiple subsets, such as provided by modern SAR satellites with small orbital tube and short revisit time. In the routine workflow, we first invert the interferogram stack for the raw phase time-series, then correct for the deterministic phase components: the tropospheric delay (using global atmospheric models or the delay-elevation ratio), the topographic residual and/or phase ramp, to obtain the noise-reduced displacement time-series. Next, we estimate the average velocity excluding noisy SAR acquisitions, which are identified using an outlier detection method based on the root mean square of the residual phase. The routine workflow includes three new methods to correct or exclude phase-unwrapping errors for two-dimensional algorithms: (i) the bridging method connecting reliable regions with minimum spanning tree bridges (particularly suitable for islands), (ii) the phase closure method exploiting the conservativeness of the integer ambiguity of interferogram triplets (well suited for highly 
A post print of a published manuscript at Computers and Geosciences

23 redundant networks), and (iii) coherence-based network modification to identify and exclude

24 interferograms with remaining coherent phase-unwrapping errors. We apply the routine

25 workflow to the Galápagos volcanoes using Sentinel-1 and ALOS-1 data, assess the qualities of

26 the essential steps in the workflow and compare the results with independent GPS measurements.

27 We discuss the advantages and limitations of temporal coherence as a reliability measure,

28 evaluate the impact of network redundancy on the precision and reliability of the InSAR

29 measurements and its practical implication for interferometric pairs selection. A comparison with

30 another open-source time series analysis software demonstrates the superior performance of the

31 approach implemented in MintPy in challenging scenarios.

33 Keywords: InSAR; time series analysis; phase-unwrapping error; phase correction; Galápagos

\section{1. Introduction}

35 Time series Interferometric Synthetic Aperture Radar (InSAR) is a powerful geodetic technique

36 to extract the temporal evolution of surface deformation from a set of repeated SAR images.

37 Accuracy and precision of the retrieved surface displacement history are limited by the

38 decorrelation of the SAR signal, the atmospheric delay and the phase-unwrapping error.

39 Decorrelation is mainly caused by changes of the surface backscatter characteristics over time

40 and by the non-ideal acquisition strategy of SAR satellites (Hanssen, 2001; Zebker and

41 Villasenor, 1992). To overcome the limitations associated with early SAR satellites, including

42 the relative long revisit time with non-regular acquisitions and the large orbit separation

43 (baseline) between repeat acquisitions, two groups of InSAR time series techniques have been

44 developed: persistent scatterer (PS) methods, which focus on the phase-stable point scatterers 
A post print of a published manuscript at Computers and Geosciences

45 with applications limited to cities and man-made infrastructures (Ferretti et al., 2001; Hooper et

46 al., 2004), and distributed scatterer (DS) methods, which relaxed the strict limit on the phase

47 stability and included areas that are affected by decorrelation through the exploitation of the

48 redundant network of interferograms. The DS methods are the focus of this paper.

50 Depending on the network of interferograms, DS methods can be divided into two categories.

51 The first category uses the network of interferograms with small temporal and spatial baselines,

52 known as small baseline subsets (SBAS) (Berardino et al., 2002; Schmidt and Bürgmann, 2003).

53 These methods solve a system of linear observation equations using least squares estimation or

$54 \quad L^{1}$-norm minimization (Lauknes et al., 2011). In cases of a non-fully connected network, singular

55 value decomposition or a regularization constraint (López-Quiroz et al., 2009) is applied to find

56 physically sound solutions. These methods require phase-unwrapped interferograms. In cases of

57 low interferometric coherence, an integer least squares estimator can be applied to the wrapped

58 interferograms, but this estimator is computationally expensive (Samiei-Esfahany et al., 2016).

60 The second category uses the network consisting of all possible interferograms with full

61 exploitation of the network redundancy (Ferretti et al., 2011; Fornaro et al., 2015; Guarnieri and

62 Tebaldini, 2008). The solution is provided by the maximum likelihood estimator with

63 performance close to the Cramér-Rao bound, the highest achievable precision (Guarnieri and

64 Tebaldini, 2007), or by eigenvalue decomposition of the covariance matrix, which has been

65 shown to be suboptimal for phase estimation (Ansari et al., 2018; Samiei-Esfahany et al., 2016).

66 These methods swap the processing order and apply the network inversion as pre-processing

67 steps for the estimation of optimal phases before phase unwrapping. 


\section{A post print of a published manuscript at Computers and Geosciences}

69 Despite the evident strengths of the full network approaches, especially the capability of phase

70 estimation on low coherent areas, they remain computationally inefficient relative to the small

71 baseline network approaches. Herein, we emphasize on the algorithmic efficiency; accordingly,

72 we implemented a weighted least squares (WLS) estimator based on SBAS method with linear

73 optimization. This process is known as phase linking or phase triangulation (Ansari et al., 2018;

74 Ferretti et al., 2011) and referred hereafter as network inversion. The precision of network

75 inversion depends on the temporal behavior of decorrelation: the small baseline network

76 approaches provide higher precision when it is fast decorrelation, while the full network

77 approaches provide higher precision when there is weak but long-term coherence (Ansari et al.,

78 2017; Samiei-Esfahany et al., 2016).

80 To separate the tropospheric delay from displacement, both PS and DS methods traditionally rely

81 on the spatio-temporal filtering of the phase time-series by taking into account their different

82 frequency characteristics in time and space domain and assuming a temporal deformation model

83 (Berardino et al., 2002; Ferretti et al., 2001), which can be unrealistic in complex natural

84 environments such as volcanic deformation. Recent developments use global atmospheric

85 models (GAMs), MERIS, MODIS or GPS wet delay (Jolivet et al., 2011; 2014; Li et al., 2009;

86 Onn and Zebker, 2006; Yu et al., 2018), or empirical correlation between stratified tropospheric

87 delay and topography (Bekaert et al., 2015; Doin et al., 2009; Lin et al., 2010) to correct

88 interferograms before network inversion. Since the contribution of tropospheric delay is a

89 deterministic component in InSAR phase observation, it is in principle preserved in the estimated

90 phase time-series and therefore can be mitigated in the time-series domain after network 
91 inversion. Similar swaps of the processing sequence have been applied to phase unwrapping

92 (Guarnieri and Tebaldini, 2008) and topographic residual correction (Fattahi and Amelung, 93 2013).

94

95 A disconnected network of interferograms with multiple interferogram subsets biases the time-

96 series estimation, especially when there is no overlap in temporal or spatial baseline among

97 interferogram subsets (Lanari et al., 2004; López-Quiroz et al., 2009). For modern SAR satellites

98 with improved orbital control and short revisit time such as Sentinel-1, the interferograms

99 network can be easily fully connected, simplifying the network inversion into an unbiased WLS

100 estimation of an overdetermined system. This robust inversion allows separating phase

101 corrections from network inversion (Pepe et al., 2011).

102

103 Here we present a new processing chain for InSAR time series analysis with phase corrections in 104 the time-series domain, in contrast to the traditional interferogram domain. We refer the time105 series domain as a series of phases indexed in time order with respect to a common reference 106 acquisition, in contrast to the interferogram domain where the phases are indexed in acquisition 107 pairs order. The basic idea is to split the time series analysis into two steps (Pepe et al., 2011): i) 108 invert network of interferograms for raw phase time-series and ii) separate tropospheric delay, 109 topographic residual, timing error and orbital error from raw phase time-series to derive the 110 displacement time-series. We also present two new methods to correct phase-unwrapping errors 111 in interferograms unwrapped by two-dimensional phase unwrapping algorithms. 
113 This paper is organized as follows. We first elaborate the theoretical basis of the weighted least

114 squares estimator and evaluate the weight functions using simulated data (section 2). The phase-

115 unwrapping error correction methods are presented in section 3. We then describe the processing

116 chain (section 4) and apply it to data on the Galápagos volcanoes (section 5), followed by a

117 discussion of results (section 6) and conclusions (section 7).

\section{2. Review of weighted least squares estimator}

\subsection{Theoretical basis}

120 We consider $N$ SAR images of the same area acquired with similar imaging geometry at times

$121\left(t_{1}, \ldots, t_{N}\right)$, which are used to generate $M$ interferograms coregistered to a common SAR

122 acquisition, corrected for earth curvature and topography and spatially phase-unwrapped,

123 referred to in the following as a stack of unwrapped interferograms. Building on Berardino et al.

124 (2002), we model the network inversion problem as a system of $M$ linear observation equations

125 with the raw phase time-series $\phi=\left[\phi^{2}, \ldots, \phi^{N}\right]^{T}$ as the vector of the $N-1$ unknown

126 parameters with reference acquisition at $t_{1} . \phi$ corresponds to the observed physical path

127 difference or range change from the SAR antenna to a ground target between each acquisition

128 and the reference one, inclusive of all systematic components including ground deformation,

129 atmospheric propagation delay and geometrical interferometric phase residuals such as those

130 caused by inaccuracy in Digital Elevation Models (DEM). For each pixel, the functional model is

131 described as:

132

$$
\Delta \phi=\boldsymbol{A} \phi+\Delta \phi_{\varepsilon}
$$


A post print of a published manuscript at Computers and Geosciences

135 where $\Delta \phi=\left[\Delta \phi^{1}, \ldots, \Delta \phi^{M}\right]^{T}$ is the interferometric phase vector with $\Delta \phi^{j}$ as the phase of the $j_{t h}$

136 interferogram, $\boldsymbol{A}$ is an $M \times(N-1)$ design matrix indicating the acquisition pairs used for

137 interferograms generation. It consists of $-1,0$ and 1 for each row with -1 for reference

138 acquisition, 1 for secondary acquisition and 0 for the rest. An example to generate $\boldsymbol{A}$ is provided

139 in the Supplementary Information section S2.1. $\Delta \phi_{\varepsilon}=\left[\Delta \phi_{\varepsilon}^{1}, \ldots, \Delta \phi_{\varepsilon}^{M}\right]^{T}$ is the vector of

140 interferometric phase residual that does not fulfill the zero phase closure of interferogram

141 triplets. It includes the decorrelation noise, phase contribution due to the change of dielectric

142 properties of ground scatterers such as soil moisture (De Zan et al., 2014; Morrison et al., 2011),

143 processing inconsistency such as filtering, multilooking, coregistration and interpolation errors

144 (Agram and Simons, 2015; Hanssen, 2001), and/or phase-unwrapping errors.

146 A fully connected network of interferograms corresponds to a full rank design matrix $\boldsymbol{A}$. Then

147 the estimation of $\phi$ can be treated as an unbiased weighted least squares inversion of an 148 overdetermined system. The solution of equation (1) can be obtained by minimizing the $L^{2}$-norm 149 of the residual phase vector $\Delta \phi_{\varepsilon}$ as:

$$
\widehat{\phi}=\operatorname{argmin}\left\|\boldsymbol{W}^{1 / 2}(\Delta \phi-\boldsymbol{A} \phi)\right\|_{2}=\left(\boldsymbol{A}^{T} \boldsymbol{W} \boldsymbol{A}\right)^{-1} \boldsymbol{A}^{T} \boldsymbol{W} \Delta \phi
$$

153 where $\hat{\phi}$ is the estimated raw phase time-series and $\boldsymbol{W}$ is an $M \times M$ diagonal weight matrix, 154 discussed in detail below. The misfit between the estimated and true raw phase time-series is 155 given as: $\hat{\phi}_{\varepsilon}=\phi-\hat{\phi}$. It's propagated from $\Delta \phi_{\varepsilon}$ through the network of interferograms. 
A post print of a published manuscript at Computers and Geosciences

157 An alternative objective function to solve equation (1) is minimizing the $L^{2}$-norm of the residual

158 of phase velocity of adjacent acquisitions (equation (16) in Berardino et al. (2002)).

159 Optimizations with both objective functions give nearly identical solutions for a fully connected

160 network. For a non-fully connected network, only the minimum-norm phase velocity gives a

161 physically sound solution (this is used by default in the software, although both objective

162 functions are supported).

163

164 For each pixel the quality of the inverted raw phase time-series can be assessed using the 165 temporal coherence $\gamma_{\text {temp }}$ (Pepe and Lanari, 2006):

$$
\gamma_{t e m p}=\frac{1}{M}\left|\boldsymbol{H}^{T} \exp [j(\Delta \phi-\boldsymbol{A} \hat{\phi})]\right|
$$

175 Since contributions of tropospheric delays, topographic residuals and/or phase ramps are 176 deterministic components in InSAR phase observations, they are preserved and therefore can be 177 mitigated in the time-series domain to obtain the displacement time-series:

$$
\phi_{\text {dis }}^{i}=\hat{\phi}^{i}-\hat{\phi}_{\text {tropo }}^{i}-\hat{\phi}_{\text {geom }}^{i}-\phi_{\text {resid }}^{i}
$$




\section{A post print of a published manuscript at Computers and Geosciences}

181 where $i \in[1, \ldots N], \hat{\phi}_{\text {tropo }}^{i}$ represents the estimated phase contribution due to the difference in

182 propagation delay through the troposphere between $t_{i}$ and $t_{1} ; \hat{\phi}_{\text {geom }}^{i}$ represents the estimated

183 geometrical range difference from radar to target caused by the non-zero spatial baseline

184 between two orbits at $t_{i}$ and $t_{1}$, including the topographic phase residual due to DEM error, phase

185 ramp due to orbital error, and possible phase ramp in range direction due to timing error of SAR

186 satellite; $\phi_{\text {resid }}^{i}$ represents the residual phase, including the residual tropospheric delay,

187 uncorrected ionospheric delay, unmodeled non-tectonic ocean tidal loads (DiCaprio and Simons,

188 2008), the remaining decorrelation noise and/or phase-unwrapping errors inherited from $\Delta \phi_{\varepsilon}$.

190 The phase introduced by orbital errors can be modeled as a linear or quadratic ramp. It can be

191 estimated and removed using GPS (Tong et al., 2013), making InSAR measurement dependent

192 on GPS. Considering its stochastic behavior and insignificant contribution to the uncertainty of

193 velocity estimation compared with the atmospheric delay for most SAR satellites with precise

194 orbits (Fattahi and Amelung, 2014), we do not correct orbital errors.

\section{$195 \quad 2.2$ Implicit assumptions}

196 The presented approach has two implicit simplifications. First, we assume that the residual term $197 \Delta \phi_{\varepsilon}$ in the phase triangulation functional model in equation (1) is zero or strictly controlled to be 198 negligible during the least squares estimation. The assumption might not be true due to the non199 conservativeness of phases in triplets of multilooked interferograms caused by the changes in the 200 scattering mechanisms. This non-conservativeness has been attributed to soil moisture variations 201 between SAR acquisitions (De Zan et al., 2014), which is especially significant for L-band (De 202 Zan and Gomba, 2018) and discussed in section 3.2 and 5.3.2. 


\section{A post print of a published manuscript at Computers and Geosciences}

204 Second, we ignored the spatial correlation of decorrelation noise between pixels. This

205 assumption is only satisfied when the SAR system resolution equals the pixel spacing. It is not

206 the case in urban areas with strong reflecting structures, or in filtered interferograms with

207 reduced resolution due to the cropped bandwidth (Agram and Simons, 2015).

\section{$208 \quad 2.3$ Choice of weight function}

209 Four different interferogram weighting strategies are implemented in the software. The first

210 strategy is uniform or no weighting, as used in the classic SBAS approach (Berardino et al.,

211 2002). In this case, the weight matrix $\boldsymbol{W}$ is equal to the identity matrix and the WLS inversion

212 simplifies into an ordinary least squares inversion. The other strategies are three different forms

213 of coherence weighting, giving observations with high coherence (low variance) more weight

214 than observations with low coherence (high variance).

215

216 In the second strategy, interferograms are directly weighted by their spatial coherence at each

217 pixel (Perissin and Wang, 2012; Pepe et al., 2015). The weight matrix takes the form:

$$
\boldsymbol{W}=\operatorname{diag}\left\{\gamma^{1}, \ldots, \gamma^{M}\right\}
$$

221 where $\gamma^{j}$ is the spatial coherence of the $j_{t h}$ interferogram.

223 In a third strategy, interferograms are weighted by the inverse of the phase variance (Tough et 224 al., 1995). The matrix takes the form: 
A post print of a published manuscript at Computers and Geosciences

$$
\boldsymbol{W}=\operatorname{diag}\left\{1 / \sigma_{\Delta \phi^{1}}^{2}, \ldots, 1 / \sigma_{\Delta \phi^{M}}^{2}\right\}
$$

228 where $\sigma_{\Delta \phi^{j}}^{2}$ is the phase variance of the $j_{\text {th }}$ interferogram calculated through the integration of the 229 phase probability distribution function (PDF). For distributed scatterers, the phase PDF is given 230 by equation (S15) in the Supplementary Information section S3.2 (Tough et al., 1995) and used

231 in the software. For persistent scatterers, the Cramér-Rao bound of variance is given directly by 232 equation (25) from Rodriguez and Martin (1992). The difference of phase PDFs between 233 distributed scatterers and persistent scatterers tends to vanish when a large number of looks is 234 applied (see supp. Fig. S1a). In practice, a lookup table is generated to facilitate the conversion 235 from spatial coherence to phase variance (see supp. Fig. S1b).

237 The fourth strategy for interferogram weighting is the nonparametric Fisher information matrix 238 (FIM), which accounts for the information loss due to noise and decorrelation, defined as 239 (Samiei-Esfahany et al., 2016; Seymour and Cumming, 1994):

$$
\boldsymbol{W}=\operatorname{diag}\left\{\frac{2 L \gamma^{1^{2}}}{1-\gamma^{1^{2}}}, \ldots, \frac{2 L \gamma^{M^{2}}}{1-\gamma^{M^{2}}}\right\}
$$

243 where $L$ is the number of independent looks used for the estimation of spatial coherence $\gamma^{j}$. Note

244 that FIM is identical to the inverse-variance matrix for persistent scatterers.

\subsection{Performance assessment of weight functions using data simulations}

246 We evaluate the performance of the different weight functions using simulated data to address

247 the question of the optimum choice of weighting for phase estimation (Cao et al., 2015). Note 


\section{A post print of a published manuscript at Computers and Geosciences}

248 that the maximum achievable precision is bounded by phase decorrelation, indicating the inverse

249 of phase variance is the optimum choice theoretically (Guarnieri and Tebaldini, 2007).

\section{$250 \quad$ 2.4.1 Simulation setting}

251 We generate the stack of interferograms for a sequential interferogram network with 10

252 connections for each image. We use the temporal and perpendicular spatial baselines from the

253 Sentinel-1 dataset of section 5. First, we specify an arbitrary temporal deformation model and

254 generate the corresponding interferometric phases (Fig. 1a). Then we simulate the spatial

255 coherence of each interferogram using a decorrelation model with exponential decay for

256 temporal decorrelation (Fig. 1b) (Hanssen, 2001; Parizzi et al., 2009; Rocca, 2007; Zebker and

257 Villasenor, 1992). Next, we simulate the corresponding decorrelation phase noise for a given

258 number of looks $L$ by generating a random number with the PDF of the interferometric phase of

259 a distributed scatterer with the given spatial coherence and number of looks and add it to the

260 noise-free phases (Fig. 1c, for $3 \times 1$ looks). The construction of the spatial coherence from the

261 decorrelation model and the simulation of the decorrelation noise are described in detail in the

262 Supplementary Information section 3. Finally, we estimate the variance of the simulated

263 interferometric phase $\sigma_{\Delta \phi^{j}}^{2}$ using windows of $5 \times 5$ pixels and transform it to equivalent spatial

264 coherence using $\gamma^{j}=1 / \sqrt{1+2 \cdot L \cdot \sigma_{\Delta \phi^{j}}^{2}}$ (Fig. 1d) (Agram and Simons, 2015). This coherence

265 is used to calculate the weight for the inversion.

\section{2.4.2 Performance assessment}

267 To quantify the performance of the time-series estimator for the four different weight functions, 268 we evaluate the difference between the inverted phase $\hat{\phi}^{i}$ and the specified, true phase $\phi^{i}$ using a 
269 root mean square error (RMSE) given as $R M S E_{\text {sim }}=\sqrt{\sum_{i=1}^{N}\left(\hat{\phi}^{i}-\phi^{i}\right)^{2} /(N-1)}$, where $N$ is the 270 number of acquisitions $(N=98)$.

271

272 Fig. 1e shows the mean RMSE for 10,000 realizations for the four different weighting 273 approaches as a function of the number of looks. To highlight differences, we also show the 274 difference in mean RMSE with respect to inverse-variance weighting (Fig. 1f). The three 275 weighted approaches outperform uniform weighting with coherence weighting performing 276 poorer than inverse-variance weighting (as shown by a positive difference in RMSE). Compared 277 to inverse-variance weighting, FIM weighting gives similar performance for more than 15 looks 278 and mixed performance for fewer looks. Similar mixed and unstable performance of FIM 279 weighting for small numbers of looks has also been observed at other simulated scenarios with 280 both higher and lower coherences (see supp. Fig. S2). This is different from a previous study 281 which supports the superiority of FIM over inverse-variance but considered only 25 looks (Fig. 8 282 of Samiei-Esfahany et al., 2016). Thus, we use the inverse of phase variance as the default 283 weight function in the software, although all four weighting strategies are supported. 
A post print of a published manuscript at Computers and Geosciences
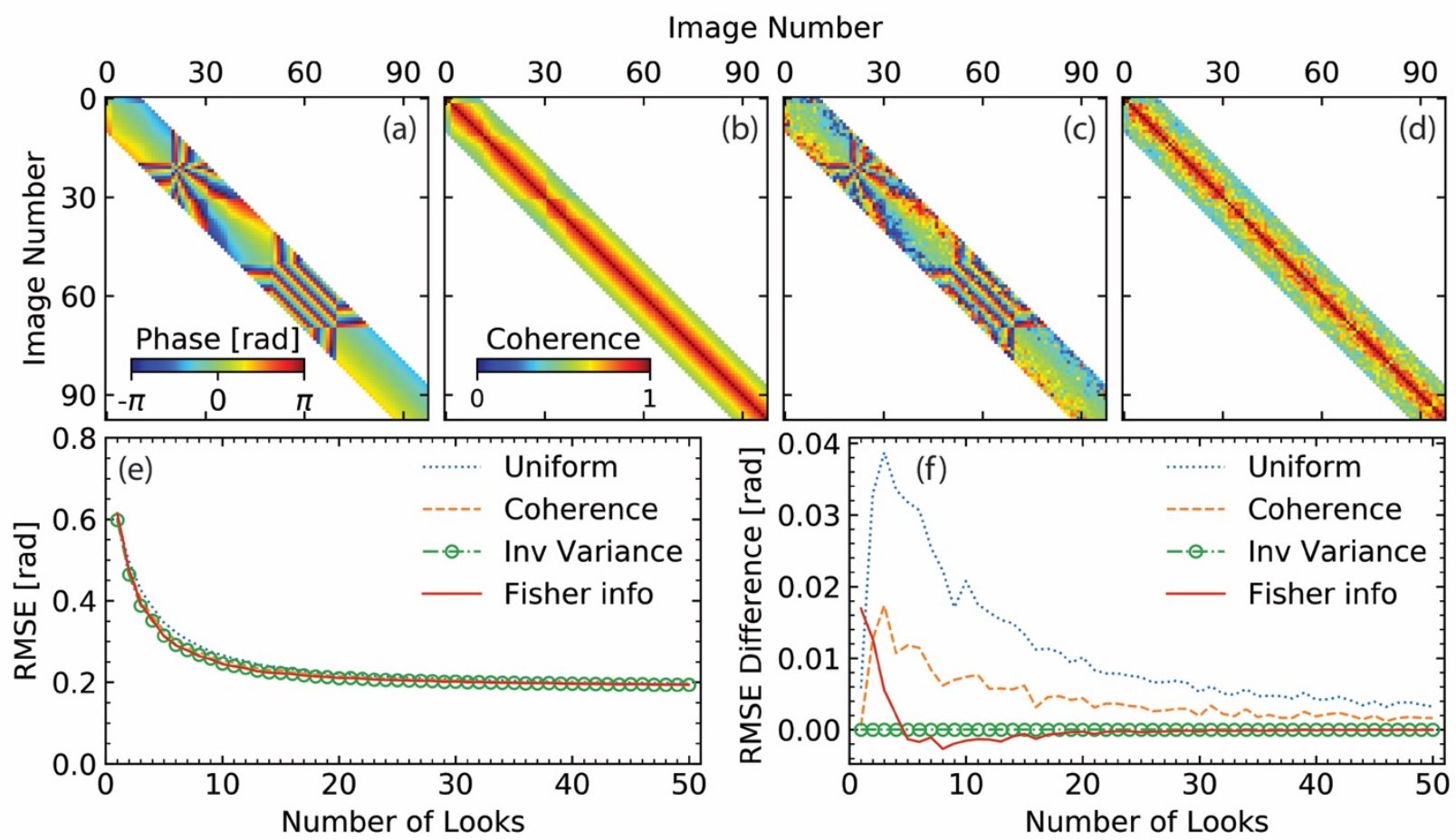

Figure 1. Simulations for weight functions performance assessment. Upper panel: a simulated

286 network of interferograms. (a-b) simulated (true) unwrapped phase and spatial coherence; (c)

287 noise-containing unwrapped phase with $L=3 \times 1$, (d) estimated coherence from the variance of

288 (c). Phase data are wrapped into $[-\pi, \pi)$ for display. (e) Mean RMSE of 10,000 realizations of

289 inverted phase time-series as a function of $L$ as the performance indicator for the four weight

290 functions. (f) Same as (e) but the difference in mean RMSE with respect to inverse-variance

291 weighting.

\section{Unwrapping error correction}

293 The inverted raw phase time-series can be potentially biased by wrong integer numbers of cycles

294 ( $2 \pi \mathrm{rad})$ added to the interferometric phase during the two-dimensional phase unwrapping, to

295 which we refer simply as unwrapping errors. Here we describe two methods to automatically

296 correct unwrapping errors using constraints from the space and time domain, respectively. 
A post print of a published manuscript at Computers and Geosciences

\subsection{Bridging of reliable regions}

In the space domain, unwrapping errors introduce phase offsets among groups of pixels that are believed to be free of relative local unwrapping errors. Such a group of pixels are referred to as a reliable region (see Chen and Zebker (2002) for a quantitative definition). These regions usually have moderate to high spatial coherence and are separated from each other due to decorrelation or high deformation phase gradients.

We assume that the phase differences between neighboring reliable regions are less than a onehalf cycle $(\pi \mathrm{rad})$ in magnitude. Then the task of unwrapping error correction is to determine the integer-cycle phase offsets to be added to each reliable region in order to align phase values among the regions. We present a bridging scheme to automatically connect reliable regions using tree searching algorithms. This is similar to region assembly in the secondary network in phase unwrapping (Carballo and Fieguth, 2002; Chen and Zebker, 2002), but in the tertiary level. To fulfill the assumption of smooth phase gradients between neighboring reliable regions, one could remove contributions from the troposphere, DEM error, deformation model, ramps before phase unwrapping and add them back in after correction. This method is particularly well suited for correcting unwrapping errors between regions separated by narrow decorrelated features such as rivers, narrow water bodies or steep topography.

\subsubsection{Algorithm}

The bridging scheme can be described as a three-step procedure for each interferogram. The first step is to identify reliable regions using the connected component information from the phase unwrapping algorithm such as SNAPHU (Chen and Zebker, 2001). Regions smaller than a preselected size are discarded. For each region, pixels on the boundaries are discarded using the 


\section{A post print of a published manuscript at Computers and Geosciences}

320 erosion in morphological image processing with a preselected shape and size. The second step is

321 to construct directed bridges to connect all reliable regions using the minimum spanning tree

322 (MST) algorithm minimizing the total bridge length. We use the breadth-first algorithm to

323 determine the order and direction (Cormen et al., 2009), starting from the largest reliable region.

324 The third step is to estimate for each bridge the integer-cycle phase offset between the two

325 regions. For that, we first estimate the phase difference as the difference in median values of 326 pixels within windows of preselected size centered on the two bridge endpoints. The integer327 cycle phase offset is the integer numbers of cycles to bring down the phase difference into [- $\pi$, $328 \pi$ ). The algorithm has the option to estimate a linear or quadratic phase ramp based on the largest 329 reliable region, which is removed from the entire interferogram before the offset estimation and 330 added back after the correction (switched off by default).

\section{$331 \quad 3.1 .2$ Simulated data}

332 We demonstrate the bridging method using a simulated interferogram of western Kyushu, Japan

333 (Fig. 2), a region with multiple islands, considering decorrelation noise, ground displacement, 334 tropospheric turbulence and phase ramps. We specify spatial coherence of 0.6 and 0.001 for 335 pixels on land and water respectively and simulate the corresponding decorrelation noise (see 336 section 2.4.1). The simulation for the other phase contributions is shown in supp. Fig. S3. We

337 wrap the simulated phase (Fig. 2a), unwrap using the SNAPHU algorithm, and apply the 338 bridging method. Fig. $2 \mathrm{~b}$ and $\mathrm{c}$ show the phase residual $\Delta \phi_{\varepsilon}^{i}$ after phase unwrapping 339 (unwrapping error) without and with unwrapping error correction, respectively. The reduction in 340 unwrapping errors (from $-2 \pi$ rad in orange shadings for the islands on the west in Fig. $2 b$ to 0 rad 341 in green shadings in Fig. 2c) demonstrates that the method works. 
A post print of a published manuscript at Computers and Geosciences

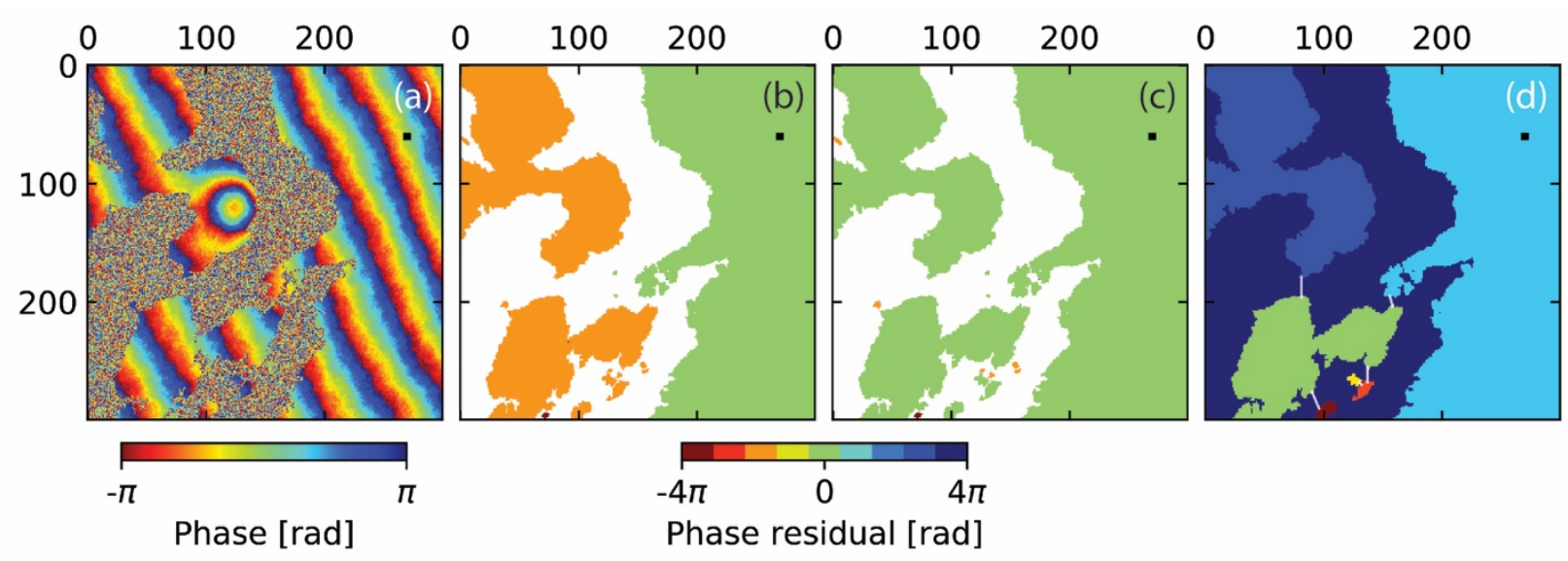

343 Figure 2. Simulation of unwrapping error correction using the bridging method. (a) Simulated

344 wrapped phase, (b and c) phase residual (unwrapping error) without and with unwrapping error

345 correction, respectively. (d) Reliable regions and bridges (white solid lines) generated based on

346 connected components from SNAPHU. White shadings in ( $b$ and $c)$ : areas not considered by the

347 connected components. Black squares represent the reference point.

\subsection{Phase closure of interferogram triplets}

349 In the time domain, unwrapping errors could break the consistency of triplets of interferometric 350 phases (Biggs et al., 2007). The closure phase is the cyclic product of the unwrapped 351 interferometric phases:

$$
C^{i j k}=\Delta \phi^{i j}+\Delta \phi^{j k}-\Delta \phi^{i k}
$$

355 where $\Delta \phi^{i j}, \Delta \phi^{j k}$ and $\Delta \phi^{i k}$ are three unwrapped interferometric phases generated from the SAR

356 acquisitions at $t_{i}, t_{j}$ and $t_{k}$. The integer ambiguity of the closure phase is given as:

$$
C_{i n t}^{i j k}=\left(C^{i j k}-\operatorname{wrap}\left(C^{i j k}\right)\right) /(2 \pi)
$$


360 where wrap is an operator to wrap the input number into $[-\pi, \pi)$. A triplet without unwrapping

361 errors has $C_{i n t}^{i j k} \equiv 0$. The number of triplets with non-zero $C_{i n t}^{i j k}$ among all triplets is given as:

$362 T_{\text {int }}=\sum_{i=1}^{T}\left(C_{i n t}^{i} \neq 0\right)$, where $T$ is the number of triplets $\left(T_{\text {int }} \leq T\right) . T_{\text {int }}$ can be used to detect

363 unwrapping errors.

365 Fig. 3 shows the characteristics of unwrapping errors in the closure phase from the Sentinel-1

366 dataset (stack of multilooked unwrapped interferograms) of section 5. The non-zero $C^{i j k}$ in Fig.

$3673 \mathrm{a}$ and $\mathrm{b}$ are caused by the interferometric phase residuals (see equation (1)), whereas the non-

368 zero $C_{i n t}^{i j k}$ in Fig. 3c are caused by unwrapping errors. Fig. $3 \mathrm{~d}$ and e show the distribution of $T_{i n t}$.

369 On Isabela island, pixels in non-vegetated area have $T_{\text {int }}=0$ (dark blue in Fig. $3 \mathrm{~d}$ ) and are free

370 of unwrapping errors; while pixels in vegetated area, such as the light-blue to green area on

371 Sierra Negra's south flank in Fig. 3d, have wide-distributed $T_{\text {int }}$ values, indicating random

372 unwrapping errors, which are difficult to correct. On Fernandina and Santiago island, most pixels

373 share the common $T_{\text {int }}$ of 229 and 576 out of 940 triplets, respectively, indicating coherent 374 unwrapping errors and can be corrected. 
A post print of a published manuscript at Computers and Geosciences
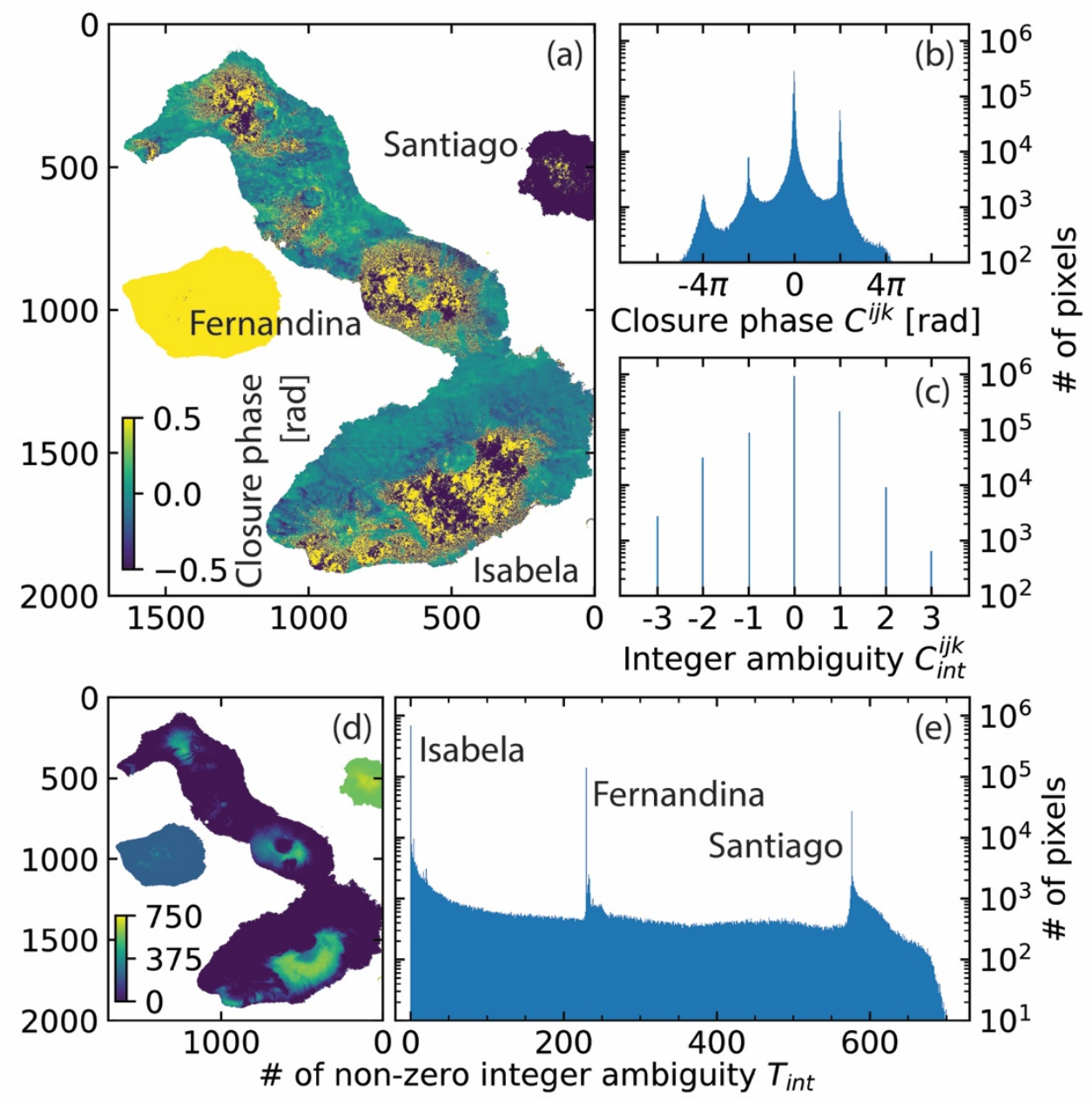

377 Figure 3. Characteristics of unwrapping errors in the closure phase. (a) Map and (b) histogram 378 of $C^{i j k}$ for the interferogram triplet generated from three Sentinel-1 images acquired at 7 March 379 2015, 19 March 2015 and 6 May 2015 from descending track 128. (c) Histogram of $C_{\text {int }}^{i j k}$ for the 380 closure phase in ( $a$ and $b)$. The non-zero $C_{i n t}^{i j k}$ are caused by unwrapping errors. (d) Map and (e)

381 histogram of $T_{\text {int }}$ (the 475 interferograms from the 98 Sentinel-1 images can be combined to 382 form 940 triplets). The spikes in (e) at 229 and 576 indicate the unwrapping error in Fernandina 383 and Santiago island, respectively. 
A post print of a published manuscript at Computers and Geosciences

385 Several attempts have been pursued to evaluate the phase unwrapping and correct the unwrapping errors using closure phase information. Hussain et al. (2016) use the closure phase to

387 adjust the cost in the three-dimensional phase unwrapping procedure iteratively. Biggs et al.

388 (2007) visually identify and correct the unwrapping errors by manually adding the integer-cycle

389 phase offsets to badly unwrapped regions of pixels. Built on this idea, we develop an algorithm

390 to automatically detect and correct the unwrapping errors in the network of interferograms.

\section{$391 \quad 3.2 .1$ Algorithm}

392 For a redundant network of interferograms, the temporal consistency of the integer ambiguities 393 of unwrapped interferometric phases can be expressed for each pixel as:

$$
\boldsymbol{C U}+(\boldsymbol{C} \Delta \varphi-\operatorname{wrap}(\boldsymbol{C} \Delta \varphi)) /(2 \pi)=0
$$

where $\boldsymbol{C}$ is a $T \times M$ design matrix of all possible interferogram triplets, $\boldsymbol{U}$ is an $M \times 1$ vector of

398 integer numbers for cycles required to meet the consistency of the interferometric phases. An can be ill-posed and does not always has a unique solution, especially when $T<M$. Thus,

401 regularization is required to obtain an optimal solution. We assume that the solution is more 402 likely to be small than large, and more likely to be sparse than dense. Accordingly, we apply the $403 \quad L^{1}$-norm regularized least squares optimization (Andersen et al., 2011; Xu and Sandwell, 2019), 404 which is also known as least absolute shrinkage and selection operator (LASSO), to obtain the 405 solution as:

$$
\widehat{\boldsymbol{U}}=\operatorname{argmin}\|\boldsymbol{C} \boldsymbol{U}+(\boldsymbol{C} \Delta \phi-\operatorname{wrap}(\boldsymbol{C} \Delta \phi)) /(2 \pi)\|_{2}+\alpha\|\boldsymbol{U}\|_{1}
$$




\section{A post print of a published manuscript at Computers and Geosciences}

409 where $\alpha=0.01$ is a nonnegative parameter for the trade-off between the $L^{1}$ and $L^{2}$-norm term,

410 with value chosen based on simulations with various values of $\alpha$ (see supp. Fig. S4). The

411 corrected unwrapped interferometric phase is given as: $\Delta \phi_{c}=\Delta \phi+2 \pi \cdot \operatorname{round}(\widehat{\boldsymbol{U}})$, where

412 round is an operator to round the input number to the nearest integer.

\section{$413 \quad 3.2 .2$ Simulated data}

414 We demonstrate the phase closure method using a simulated interferogram stack for one pixel

415 (Fig. 4). We first simulate the decorrelation noise and ground deformation (see section 2.4.1) for 416 an interferogram network with 5 sequential connections using the temporal and perpendicular

417 spatial baselines from the Sentinel-1 dataset of section 5 . Then we randomly select $20 \%$ of the 418 interferograms to add unwrapping errors with randomly selected cycles (maximum of 2) of 419 magnitude and randomly selected sign. Next, we apply the phase closure method and compare 420 the unwrapping errors before and after the correction, as shown in orange and blue bars in Fig. $4214 a$, respectively. The method decreases the number of interferograms affected by unwrapping 422 errors from $20 \%$ to $2 \%$ and reduces the magnitude of the remaining unwrapping errors (Fig. $4 \mathrm{a}$ ).

423 We note that the method could potentially introduce new unwrapping errors to the unwrapped 424 interferograms (blue bars in Fig. 4a where there is no orange bar).

426 We evaluate the performance of the phase closure method by comparing the input and output 427 percentages of interferograms with unwrapping errors (before and after correction), considering 428 different input percentages and redundancies of the interferogram network. Fig. 4b shows for 429100 realizations the mean output percentage after correction versus the input percentage for 430 networks with 3, 5 and 10 sequential interferograms. For 5 connections (orange dots in Fig. 4b), 
A post print of a published manuscript at Computers and Geosciences

431 the method fully corrects unwrapping errors if there are less than $20 \%$ of interferograms affected;

432 then the improvement slows down with the increasing input percentage until it reaches a turning

433 point of $35 \%$, beyond which the improvement is marginal. The maximum input percentages with

434 full correction for 3,5 and 10 connections are at 5, 20 and 35\%, respectively, indicating better

435 performance for more redundant networks. Fig. 4c shows the performances for 5 connections

436 network with maximum of 2, 5 and 10 cycles of unwrapping errors. The similarity before $30 \%$

437 shows that the method is robust for various magnitudes of unwrapping errors. Thus, we conclude

438 that the phase closure method is suitable for highly redundant networks of interferograms with

439 not too many unwrapping errors.

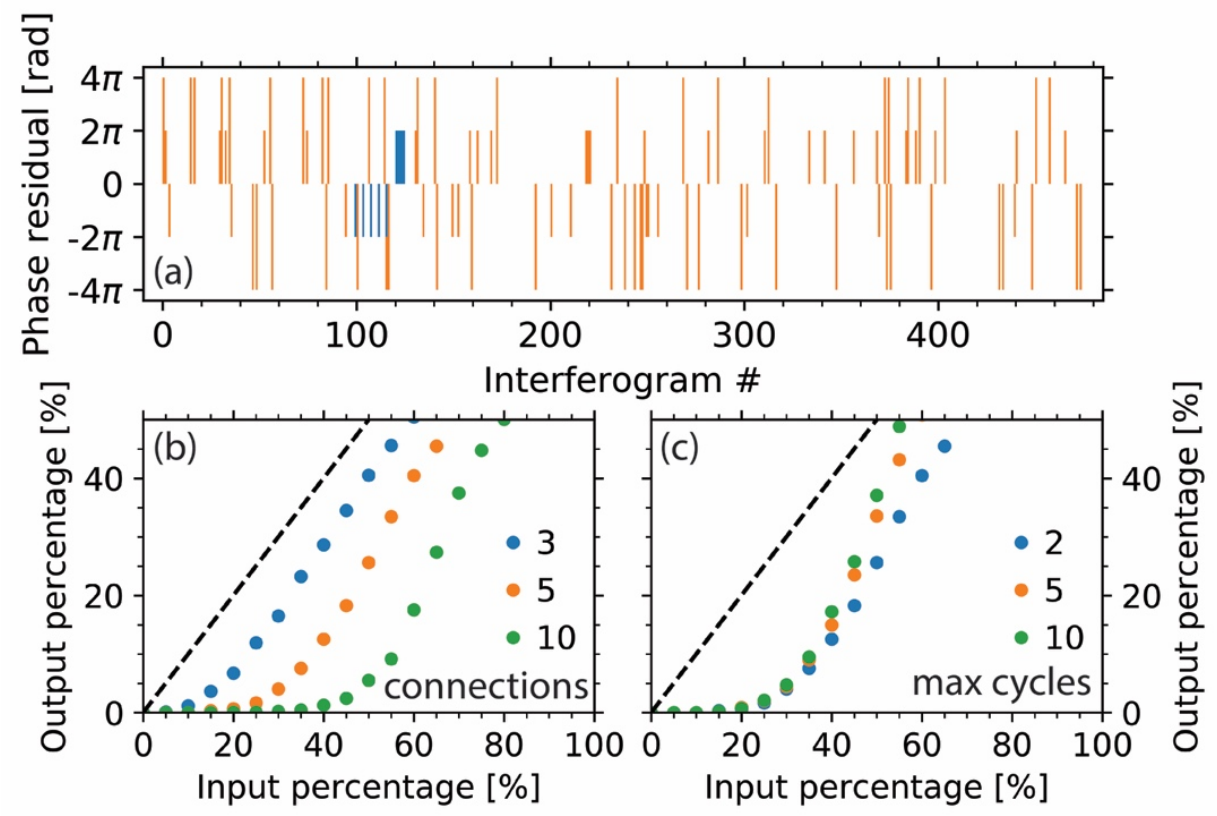

Figure 4. Simulations of unwrapping error correction using the phase closure method. (a)

442 Unwrapping errors in interferograms before (orange bars, account for 20\%) and after 443 correction (blue bars, account for 2\%). A network of interferograms with 5 sequential 444 connections is used. A maximum of 2 cycles of unwrapping errors are added randomly. (b) Mean 445 output percentage of 100 realizations of interferograms with unwrapping errors versus the input 
A post print of a published manuscript at Computers and Geosciences

446 percentage, with a fixed maximum of 2 cycles of unwrapping errors and color coded by network

447 redundancy. (c) Same as (b) but with a fixed network of 5 connections and color coded by

448 maximum unwrapping error magnitudes.

\section{4. Workflow of InSAR time series analysis}

450 We have implemented a generic routine processing workflow for InSAR time series analysis

451 from a stack of unwrapped interferograms to displacement time-series (Fig. 5). The workflow

452 consists of two main blocks: (i) correcting unwrapping errors and inversion for the raw phase

453 time-series (blue ovals in Fig. 5), and (ii) correcting for phase contributions from different

454 sources to obtain the displacement time-series (green ovals in Fig.5). It includes some optional

455 steps, which are switched off by default (marked by dashed boundaries in Fig. 5), here we

456 present the workflow in its most complete form. Configuration parameters for each step are

457 initiated with default values in a customizable text file (link on GitHub).

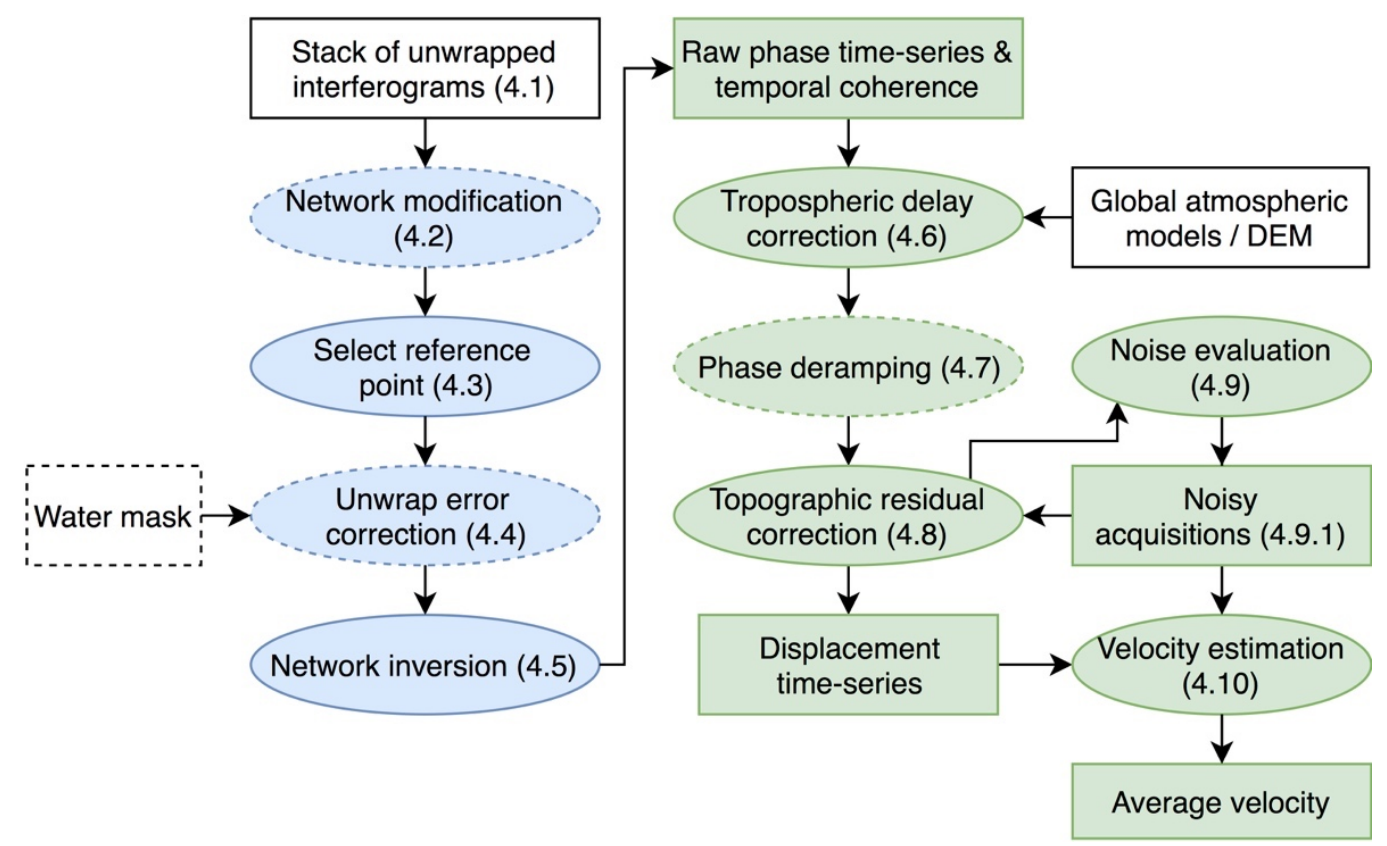


A post print of a published manuscript at Computers and Geosciences

459 Figure 5. Routine workflow of InSAR time series analysis. Blue ovals: steps in the interferogram

460 domain including unwrapping error correction and network inversion; green ovals: steps in the

461 time-series domain including phase corrections for the tropospheric delay, phase ramps, and

462 topographic residuals. White rectangles: input data. Green rectangles: output data. Optional

463 steps/data are marked by dashed boundaries.

\section{4.1 Starting point: Stack of unwrapped interferograms}

465 As described above, the starting point is a stack of phase-unwrapped interferograms coregistered

466 to a common SAR acquisition, corrected for earth curvature and topography. We currently

467 support interferogram stacks produced by ISCE, GAMMA and ROI_PAC software (Rosen et al.,

468 2004; Rosen et al, 2012; Werner et al., 2000).

\section{$469 \quad 4.2$ Network modification}

470 In order to exclude outliers affected by coherent pixels with unwrapping errors, the software

471 provides network modification to exclude affected interferograms if the spatially averaged

472 coherence for an area of interest falls below a predefined threshold value (switched off by

473 default). This is similar to Chaussard et al. (2015) excluding interferograms with a low

474 percentage of high coherent pixels. An extra constraint could be applied to keep those

475 interferograms if they are part of the MST network providing the maximum spatially averaged

476 coherence (Perissin and Wang, 2012) to ensure a fully connected network (switched on by

477 default). The approach is referred to as coherence-based network modification. This is based on

478 the empirical observation that reliable regions with unwrapping errors are usually surrounded by

479 decorrelated areas. The default area of interest is all pixels on land, a customized area of interest

480 including the decorrelated areas around the reliable regions is usually more effective. The

481 software also supports other approaches for network modification, such as thresholds of the 
A post print of a published manuscript at Computers and Geosciences

482 temporal and spatial baselines, maximum number of connections for each acquisition, and 483 exclusion of specific acquisitions, interferograms.

\section{$484 \quad 4.3$ Reference selection in space}

485 The reference pixel is selected randomly among the pixels with high average spatial coherence 486 ( $\geq 0.85$ by default) or can be specified using prior knowledge of the study area. The reference 487 pixel should be (i) located in a coherent area; (ii) not affected by strong atmospheric turbulence 488 such as ionospheric streaks and (iii) close to and with similar elevation as the area of interest to 489 minimize the impact of the spatially correlated atmospheric delay. For example, Chaussard et al. 490 (2013) studied volcano deformation using reference points on inactive, neighboring volcanoes.

\section{$491 \quad 4.4$ Unwrapping error correction}

492 Three methods are available to possibly detect and correct unwrapping errors in the stack of 493 interferograms. The first method is bridging as described in section 3.1. This method is well 494 suited for unwrapping errors occurred among islands or on areas separated by steep topography. 495 The second method is based on the phase closure as described in section 3.2. It's well suited for 496 unwrapping errors in a highly redundant network of interferograms. Both methods are operated 497 in the region level, thus are efficient. The third approach is to apply both methods, bridging 498 followed by phase closure, as they exploit aspects of unwrapping errors in space and time 499 domain, respectively. The default is no unwrapping error correction.

\section{$500 \quad 4.5$ Network inversion}

501 The raw phase time-series is solved by minimizing the interferometric phase residual $\Delta \phi_{\varepsilon}$. Then,

502 the temporal coherence is computed based on equation (3) and used to generate a temporal 503 coherence mask for pixels with reliable time-series estimation with a predefined threshold $(0.7$ 


\section{A post print of a published manuscript at Computers and Geosciences}

504 by default). Pixels in shallow and water bodies are masked out if shallow mask and water body 505 mask are available.

\subsubsection{Phase masking}

507 In order to exclude outliers affected by decorrelation, the software provides masking options 508 (switched off by default) based on the spatial coherence (default threshold of 0.4 ) or using the 509 connected component information from phase unwrapping. Note that masking based on spatial 510 coherence is equivalent to weighting with a step function.

512 After masking, the pixels may have different numbers of interferograms. We use not only the 513 pixels that are coherent in all interferograms (Agram and Simons, 2015), but relax the pixel 514 selection criterion and also use pixels with fewer interferograms as long as a predefined

515 minimum number of interferograms is available for each SAR acquisition (1 by default). Note

516 that with this pixel selection strategy after masking, the network inversion result is not sensitive

517 to the few very low coherent interferograms in a redundant network, giving robust and consistent 518 spatial coverage.

\section{$519 \quad 4.6$ Tropospheric delay correction}

520 Two different approaches for tropospheric delay correction are available. In the first approach,

521 the tropospheric delay is estimated using Global Atmospheric Models (GAMs). The estimated 522 relative double path tropospheric delay at $t_{i}$ between a given pixel $p$ and a reference pixel is 523 given in radians as:

$$
\hat{\phi}_{\text {tropo }}^{i}(p)=\left(\delta L_{p}^{i}-\delta L_{p}^{1}\right) \frac{4 \pi}{\lambda}-\left(\delta L_{r e f}^{i}-\delta L_{r e f}^{1}\right) \frac{4 \pi}{\lambda}
$$




\section{A post print of a published manuscript at Computers and Geosciences}

527 where $i \in[1, \ldots N], \delta L_{x}^{i}$ is the integrated absolute single path tropospheric delay at $t_{i}$ on pixels $x$

528 in meters in satellite line-of-sight (LOS) direction $\left(\delta L_{p}^{1}\right.$ for $\left.t_{1}\right)$ and $\lambda$ is the radar wavelength in

529 meters. The supported datasets include ERA-5 and ERA-Interim from European Center for

530 Medium-Range Weather Forecast, NARR (North American Regional Reanalysis) from NOAA

531 and MERRA (Modern-Era Retrospective Analysis) from NASA (applied by default, using

532 PyAPS software from Jolivet et al. (2011; 2014)).

533

534 The second approach is based on the empirical linear relationship between the InSAR phase

535 delay and elevation (Doin et al., 2009) which in areas with strong topographic variations

536 sometimes outperforms corrections using GAMs. On the other hand, the empirical approach

537 cannot distinguish between the stratified tropospheric delay and the ground deformation 538 correlated with topography such as at volcanoes.

\subsection{Phase deramping}

540 Phase ramps are caused by residual tropospheric and ionospheric delays and to a lesser extent, by

541 orbital errors. For long spatial wavelength deformation signals such as interseismic deformation,

542 ramps should not be removed. Instead, physical and statistical approaches should be applied to

543 correct the ionospheric delay (Fattahi et al., 2017; Gomba et al., 2016; Liang et al., 2018) and/or

544 assess the measurement uncertainties (Fattahi and Amelung, 2014; 2015; Fattahi et al., 2017).

545 For short spatial wavelength deformation signals such as volcanic deformation, landslides, and

546 urban subsidence it is recommended to estimate and then to remove linear or quadratic ramps

547 from the displacement time-series at each acquisition on the reliable pixels (default is no ramp 548 removal). 
A post print of a published manuscript at Computers and Geosciences

549

550

551

552

553

554

555

556

557

558

559

560

561

562

563

564

565

566

567 demonstrated in supp. Fig. S5 (default is no step function with $N_{p o l y}=2$ ).

\subsection{Topographic residual correction} polynomial order $N_{p o l y}$. The DEM error $z_{\varepsilon}$ for each pixel is then given by:

The systematic topographic phase residual caused by a DEM error is estimated based on the proportionality with the perpendicular baseline time-series (Fattahi and Amelung, 2013). The original method assumes a cubic temporal deformation model, which is not able to capture highfrequency displacement components, such as offsets caused by earthquakes or volcanic eruptions. The software provides options to account for permanent displacement jumps using step functions (Hetland et al., 2012) and to generalize polynomial functions with a user-defined

where $i \in[1, \ldots N], B_{\perp}^{i}$ is the perpendicular baseline between $t_{i}$ and $t_{1}, r$ is the slant range between the target and the radar antenna, $\theta$ is the incidence angle, $H\left(t_{i}-t_{l}\right)$ is a Heaviside step function centered at $t_{l}, I_{s}$ is a set of indices describing offsets at specific prior selected times. $z_{\varepsilon}$, $c_{k}$ and/or $s_{l}$ are the unknown parameters, which can be estimated by minimizing the $L^{2}$-norm of residual phase time-series $\phi_{\text {resid }}=\left[\phi_{\text {resid }}^{1}, \ldots, \phi_{\text {resid }}^{N}\right]^{T}$. An example design matrix and the numerical solution of least squares estimation are provided in the Supplementary Information section 2.3. The necessity of the step function(s) in the presence of deformation jump(s) is 568

569 As we are interested in the estimation of $z^{\varepsilon}$, the assumed deformation model does not need to be 570 a comprehensive representation of the deformation processes. Note, however, that equation (13) 
571 offers the possibility to parameterize the geophysical processes using more complex models, e.g.

572 using the regularization functions from Hetland et al. (2012).

\section{$573 \quad 4.9$ Residual phase for noise evaluation}

574 The estimate of residual phase $\hat{\phi}_{\text {resid }}$, a by-product of equation (13), is the phase component that 575 can neither be corrected nor be modeled as ground deformation, thus, is used to characterize the 576 noise level of the InSAR time-series. For each SAR acquisition, we compute the root mean 577 square (RMS) of the residual phase as:

$$
R M S^{i}=\sqrt{\frac{1}{N_{\Omega}} \sum_{p \in \Omega}\left(\hat{\phi}_{\text {resid }}^{i}(p) \cdot \frac{\lambda}{-4 \pi}\right)^{2}}
$$

581 where $i=[1, \ldots, N], \widehat{\phi}_{\text {resid }}^{i}(p)$ represent the residual phase at $t_{i}$ for pixel $p, \Omega$ is the set of 582 reliable pixels selected based on temporal coherence during the network inversion with the total 583 number of $N_{\Omega}$. Due to the inadequate knowledge of the long spatial wavelength phase

584 components in $\widehat{\phi}_{\text {resid }}$, we focused on the noise evaluation of the short spatial wavelength phase 585 components only, including residual tropospheric turbulence, uncorrected ionospheric 586 turbulence, and remaining decorrelation noise. Therefore, we remove a quadratic ramp from the 587 residual phase of each acquisition before calculating the RMS (Lohman and Simons, 2005; 588 Sudhaus and Jónsson, 2009).

\subsubsection{Identifying noisy SAR acquisitions}

590 Assuming the residual tropospheric delay in $\hat{\phi}_{\text {resid }}$ is stochastic and Gaussian distributed in time 591 (Fattahi and Amelung, 2015), we can treat the noisy SAR acquisitions contaminated by severe 592 atmospheric turbulence as outliers. Following Rousseeuw and Hubert (2011), we calculate the 
A post print of a published manuscript at Computers and Geosciences

593 median absolute deviation (MAD) value and mark a SAR acquisition as noisy if its RMS value is

594 larger than the predefined cutoff ( 3 MADs by default giving $99.7 \%$ confidence). Note that we

595 assume a zero-mean value for the distribution considering the positive nature of RMS. The

596 automatically identified noisy acquisitions will be excluded in the topographic residual

597 estimation (during re-run) and velocity estimation.

\section{$598 \quad 4.9 .2$ Selecting the optimal reference date}

599 The SAR acquisition with the smallest RMS value can be interpreted as the date with minimum 600 atmospheric turbulence and is used as the reference date. We note that changing the reference 601 date is equivalent to adding a constant to the displacement time-series, which does not change 602 the velocity or any other information derived from the displacement time-series.

\subsection{Average velocity estimation}

604 For applications with interest on the deformation rate, the velocity $v$ is estimated as the slope of 605 the best fitting line to the displacement time-series, given as $\phi_{d i s}^{i} \cdot \lambda /(-4 \pi)=v \cdot t_{i}+c, i=$ $6061, \ldots, N$, where $c$ is an unknown offset constant. Noisy SAR acquisitions are excluded by default 607 during the estimation. The standard deviation of the estimated velocity is given by equation (10) 608 from Fattahi and Amelung (2015).

\section{5. Application to Galápagos volcanoes, Ecuador}

610 We apply the routine workflow outlined in the previous section to the western Galápagos

611 Islands, Ecuador, located around $1000 \mathrm{~km}$ west of Ecuador mainland (Fig. 6 inset). We consider

612 interferogram stacks from the Sentinel-1 and ALOS-1 satellite. For Sentinel-1 (we consider the

613 December 2014 to June 2018 period) we use the stack Sentinel processor (Fattahi et al, 2016) 


\section{A post print of a published manuscript at Computers and Geosciences}

614 within ISCE (Rosen et al, 2012) for processing the stack of interferograms; we pair each SAR

615 image with its five nearest neighbors back in time (sequential network); we multilook each

616 interferogram by 15 and 5 looks in range and azimuth direction respectively, filter using a

617 Goldstein filter with a strength of 0.2 (configuration file). For ALOS-1 we use ROI_PAC (Rosen

618 et al., 2004) for processing the stack of interferograms; we select interferometric pairs with small

619 temporal (1800 days) and spatial baselines $(1800 \mathrm{~m})$ and with over $15 \%$ of Centroid doppler

620 frequency overlap in azimuth direction; we multilook each interferogram by 8 and 16 looks in

621 range and azimuth direction respectively, filter using a Goldstein filter with a strength of 0.5 and

622 an adaptive smoothing with a width of 4 pixels (configuration file). We remove the topographic

623 phase component using SRTM DEM (SRTMGL1, 30m, 1 arc second with void-filled; Farr et

624 al., 2007). The interferograms are phase-unwrapped using the minimum cost flow method (Chen

625 and Zebker, 2001). In the routine workflow for the Sentinel-1 dataset we correct unwrapping

626 errors using the bridging and phase closure method. In the routine workflow for the ALOS-1

627 dataset we exclude interferograms using coherence-based network modification with a

628 customized area of interest (blue rectangle in Fig. 10b) and correct unwrapping errors using the

629 bridging method. We remove linear phase ramps from both datasets.

631 The Islands host seven active volcanoes characterized by large summit calderas with several $\mathrm{km}$

632 radii and by distinguished nonlinear deformation behavior. The surface coverage ranges from

633 bare lava flows to dense vegetation. We discuss observations of Sierra Negra, Cerro Azul,

634 Alcedo, Wolf and Fernandina volcanoes. Sierra Negra erupted in 26 June 2018, Wolf volcano in

635 May 2015 and Fernandina volcano in September 2017 and June 2018. 
637 Products of the routine workflow include the mean LOS velocity (Fig. 6) and the displacement 638 time-series (Fig. 7, shown for Fernandina island only). The center of Sierra Negra caldera 639 uplifted at a mean rate of $60 \mathrm{~cm} / \mathrm{yr}$ (Fig. 6) but the uplift rate varied with time (Fig. 8). The 640 deformation at Cerro Azul volcano was caused by a sill intrusion in March 2017 (Bagnardi and 641 Hooper, 2018).

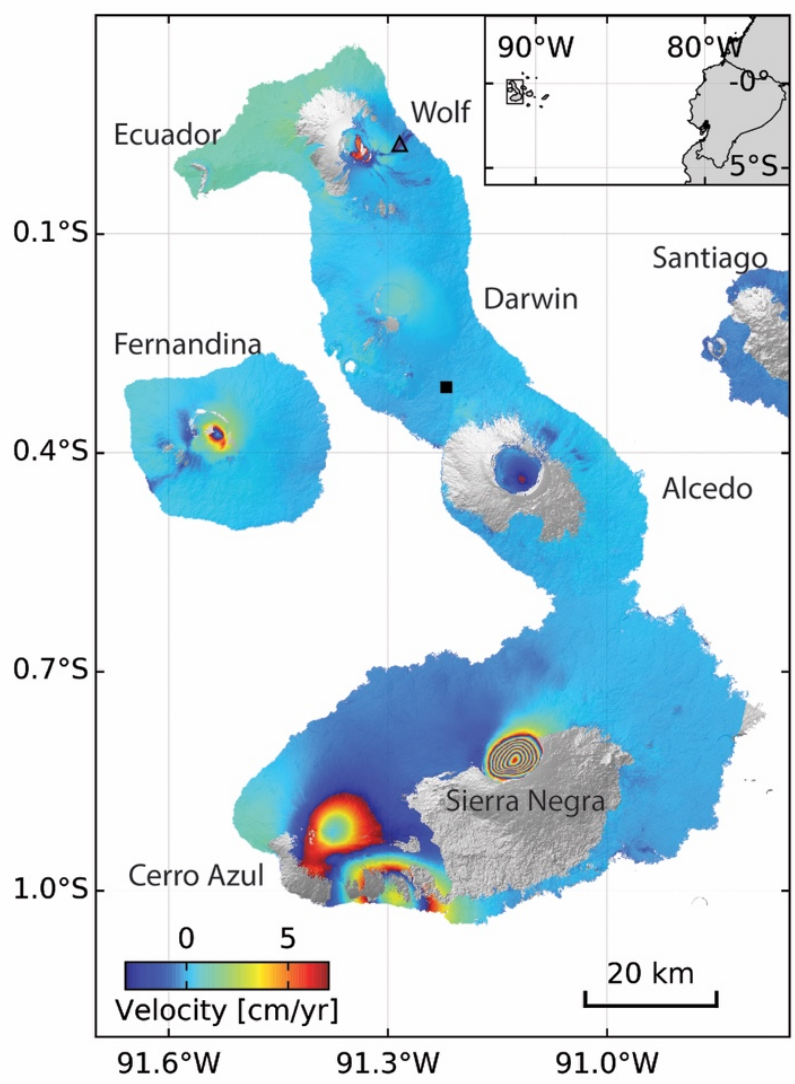

643 Figure 6. Mean LOS velocity at Isabela, Fernandina, and Santiago (main image), the 644 westernmost islands in the Galápagos archipelago (inset). The velocity is estimated from 98 645 Sentinel-1 descending track 128 SAR acquisitions from December 2014 to 19 June 2018 and 646 wrapped into $[-3,7) \mathrm{cm} / y$ r for display so that one color-cycle represents $10 \mathrm{~cm} / \mathrm{yr}$ displacement 647 velocity. Black square represents the reference point. Black triangle indicates the location of the 648 pixel covered by the lava flow of the 2015 Wolf eruption used in Fig. $15 \mathrm{~b}$ and c. Dark blue in 649 Santiago island indicates biased velocity estimation caused by remaining unwrapping errors. 
A post print of a published manuscript at Computers and Geosciences

650 The southeast part of the caldera of Volcán Alcedo has been subsiding at a rate of $-3.1 \mathrm{~cm} / y \mathrm{r}$.

651 The center of Fernandina caldera uplifted by $14 \mathrm{~cm}$ before the September 2017 eruption,

652 subsided during the eruption and uplifted by $35 \mathrm{~cm}$ until the June 2018 eruption (Fig. 7).

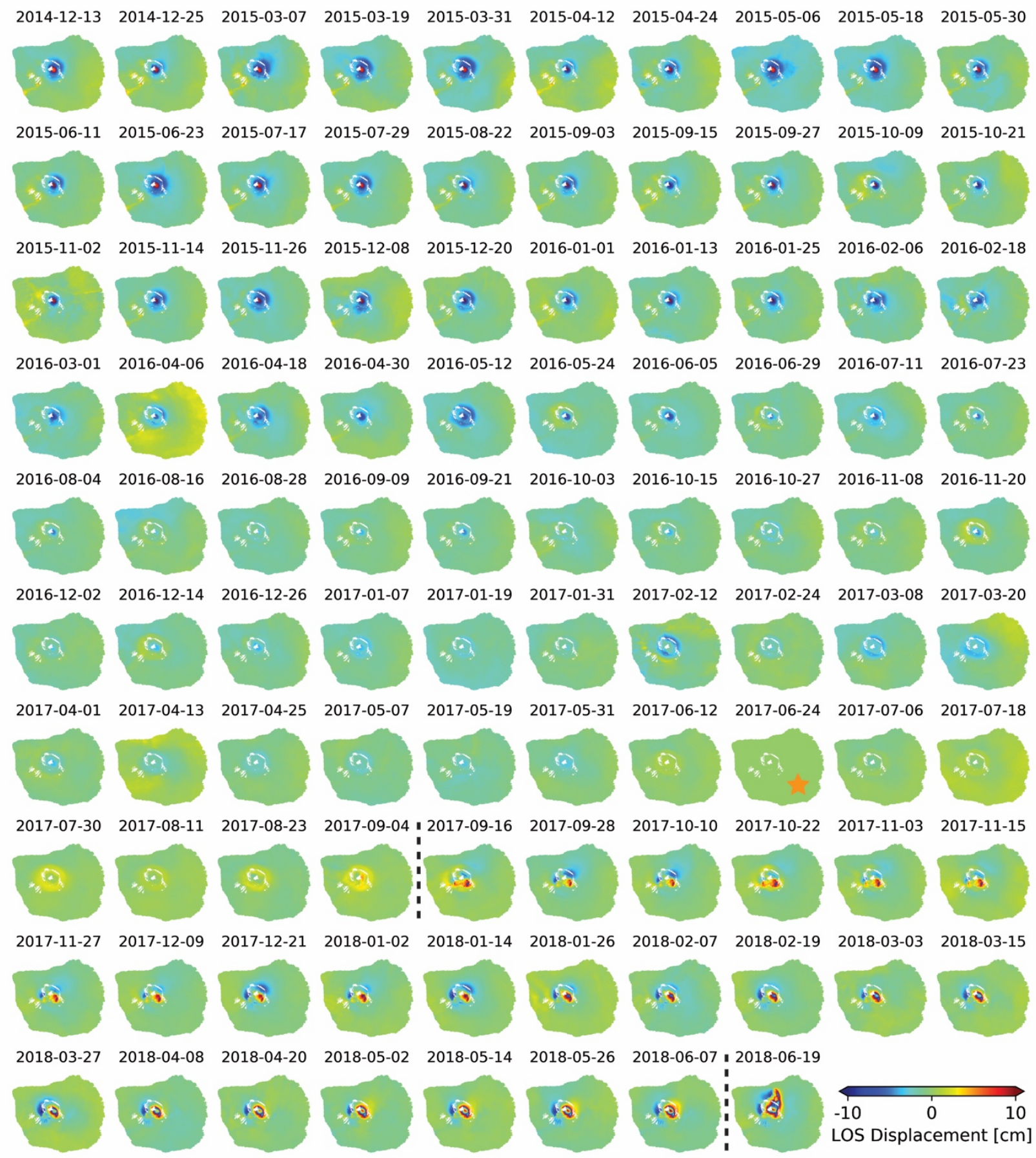


A post print of a published manuscript at Computers and Geosciences

654 Figure 7. Displacement time-series on Fernandina volcano with Sentinel-1 data. Dashed lines:

655 eruption events on September 2017 and June 2018. Orange star: automatically selected

656 reference date. The reference point is on Isabela island (black square in Fig. 6). Data are

657 wrapped into [-10, 10) cm for display.

$658 \quad 5.1$ Comparison with GPS

659 To validate the InSAR measurements we use the continuous GPS measurements at stations in the 660 Sierra Negra caldera (circles in Fig. 8a; Blewitt et al., 2018). All three GPS components in east, 661 north and vertical directions are used to project displacements into InSAR LOS direction. Both 662 InSAR and GPS time-series are referenced to station GV01 in space and a common reference 663 date in time. The InSAR data for each GPS point is obtained by linear interpolation (InSAR pixel 664 size is $64 \times 70 \mathrm{~m}^{2}$ ). The InSAR and GPS total displacements for the period of interest (Fig. 8a) 665 and the displacement time-series (Fig. 8b) agree very well, except for GV10 discussed below. To 666 quantify the agreement, we assume the GPS time-series as truth and compute the coefficient of 667 determination $R^{2}$ between InSAR time-series and GPS time-series and the RMSE given as:

668

669

$$
R M S E_{\text {InSAR }}=\sqrt{\sum_{i=1}^{N_{\text {comm }}}\left(d_{\text {InSAR }}^{i}-d_{G P S}^{i}\right)^{2} /\left(N_{\text {comm }}-1\right)}
$$

671 where $d_{I n S A R}^{i}=\phi_{d i s}^{i} \cdot \frac{\lambda}{-4 \pi}$ and $d_{G P S}^{i}$ are the InSAR and GPS time-series in LOS direction, 672 respectively, at the $i_{t h}$ common date. $N_{\text {comm }}$ is the total number of common dates.

674 The temporal coherence at the GPS stations varies from 0.96 to 1.0 (Fig. 8b) indicating reliable 675 InSAR measurements at these locations (except GV10). The $R^{2}$ at the GPS stations are 1.0 and 
676 the RMSE varies from 0.5 to $1.8 \mathrm{~cm}$ (Fig. 8b), confirming the good agreement of the two

677 measurements. The exception is station GV10 ( $R^{2}$ of 0.72 and RMSE of $\left.3.9 \mathrm{~cm}\right)$, which is

678 eliminated during posterior quality assessment due to low temporal coherence of 0.64 (below the

679 threshold of 0.7 ). This station is located in a more densely vegetated area outside the caldera on

680 the rim where decorrelation due to vegetation affects the interferometric coherence (see supp.

681 Fig. S6).

682

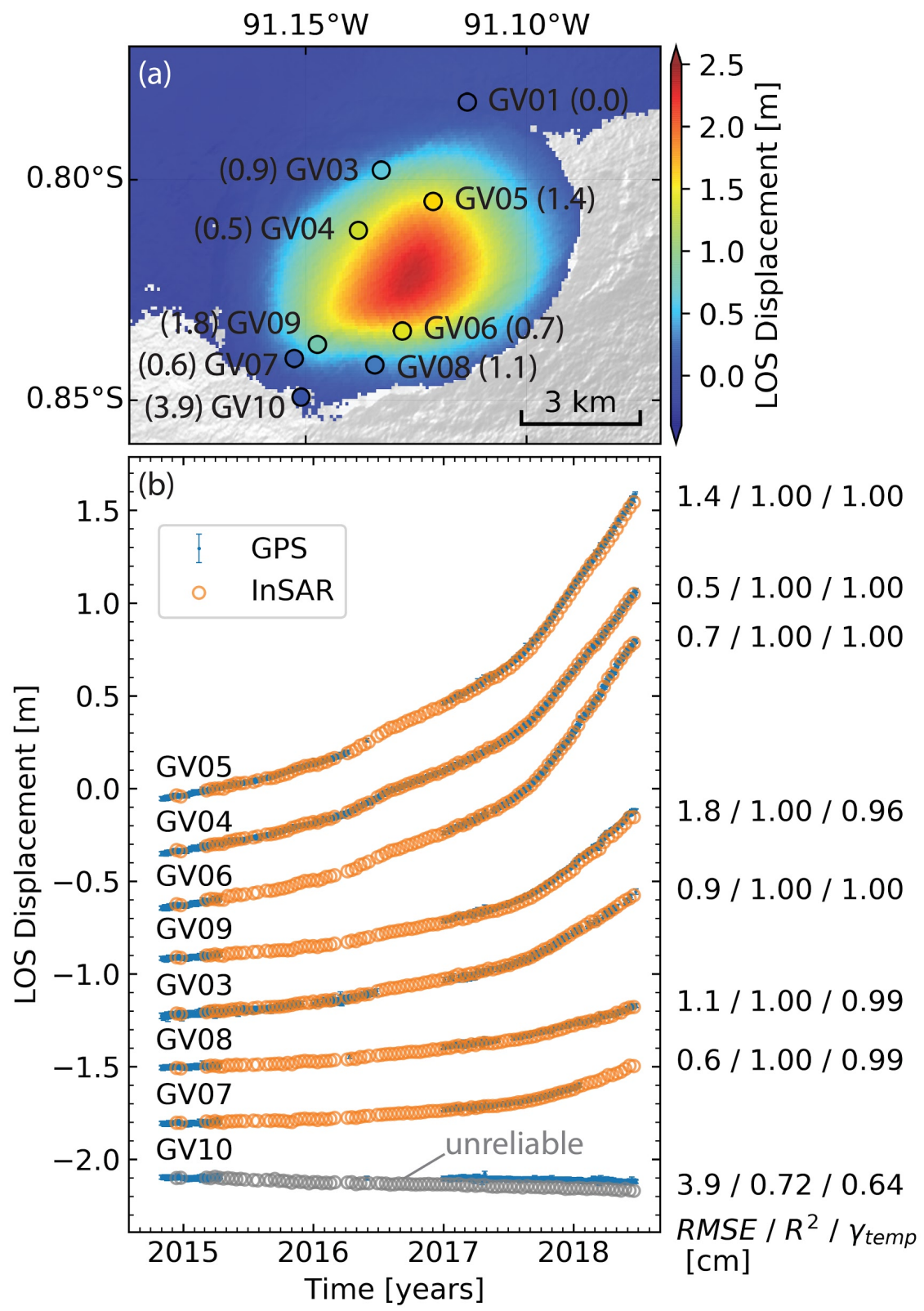


A post print of a published manuscript at Computers and Geosciences

683 Figure 8. Comparing InSAR with GPS. (a) Total displacements in LOS direction for Sierra 684 Negra caldera from InSAR and GPS during 13 December 2014 - 19 June 2018. Circles: GPS 685 stations colored by displacement. Positive displacements indicate motion towards the satellite. 686 (b) Displacement time-series from InSAR and GPS relative to GV01 (shifted for display). Blue 687 GPS error bars: three sigma uncertainties (in LOS direction propagated from the uncertainties 688 in east, north and up direction). 12 April 2015 is selected as the common reference because this 689 SAR acquisition is characterized by small residual phase RMS. Gray circles: unreliable InSAR 690 time-series with temporal coherence less than 0.7 (masked out by default).

\subsection{Assessment of unwrapping error correction}

692 The islands of Fernandina and Santiago exhibit unwrapping errors relative to Isabela island due

693 to the water separation. The unwrapping errors are represented by the low temporal coherence of 694 about 0.49 and 0.07 for Fernandina and Santiago with Sentinel-1 dataset, respectively (pixel A 695 and B in Fig. 9a). Since there is no indication of localized submarine deformation between 696 Isabela and Fernandina or between Isabela and Santiago during the time period of Sentinel-1

697 dataset, we believe the phase differences among the three islands fulfill the bridging assumption 698 (less than $\pi \mathrm{rad}$ in magnitude). Thus, we applied the bridging method followed by the phase 699 closure method to correct the potential unwrapping errors in the interferogram stack (Fig. 9). The 700 bridging method leads to increased temporal coherence of 0.96 and 0.55 at these two points, 701 respectively (Fig. 9b). The phase closure method leads to further increased temporal coherence 702 of 1.00 and 1.00 , respectively (Fig. 9c).

703

704 We note that for Santiago, however, the phase closure method did not fully correct the large 705 amount of unwrapping errors, resulting in a biased average velocity estimation of $-0.5 \mathrm{~cm} / \mathrm{yr}$ 
706 (Fig. 6). This is due to the assumption of sparse unwrapping errors in the phase closure method,

707 which is not the case for the Sentinel-1 dataset in Santiago: 576 out of 940 interferogram triplets

708 have non-zero integer ambiguity (Fig. 3e). Conversely temporal coherence after the phase

709 closure correction can be partly biased.
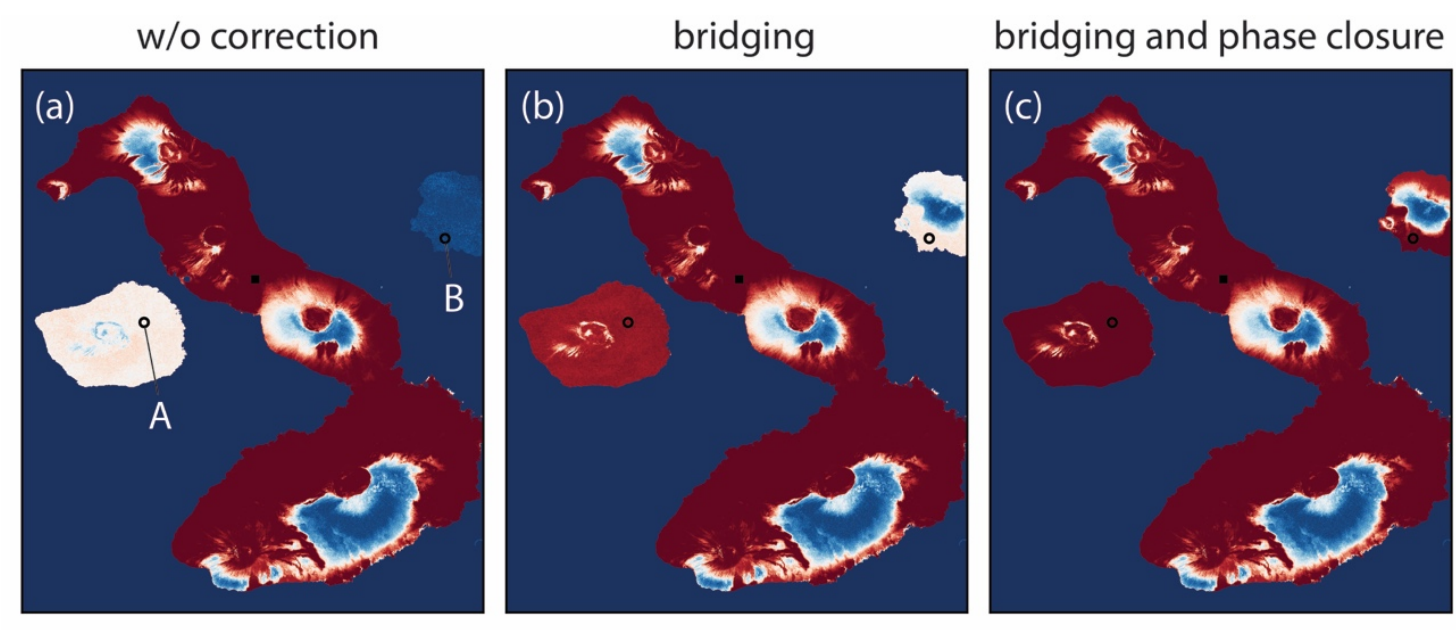

710

Figure 9. Assessment of unwrapping error correction. Temporal coherence of the Sentinel-1

712 dataset from the network inversion of the interferogram stack (a) before the unwrapping error

713 correction, (b) after the unwrapping error correction with bridging and (c) with bridging and

714 phase closure. Black squares indicate the reference point.

\section{$715 \quad 5.3$ Assessment of network inversion}

\section{$716 \quad 5.3 .1$ Temporal coherence}

717 The quality of the network inversion can be evaluated posteriorly using the temporal coherence.

718 In Fig. 10, we compare for the ALOS-1 dataset the temporal coherence obtained by inverting a

719 network of small baseline interferograms using uniform weighting (classic SBAS; Fig. 10a-c)

720 with that obtained by inverting the network after coherence-based network modification (an

721 option of the routine workflow) using inverse-variance weighting (Fig. 10d-f). The first approach

722 assumes an oversimplified linear relationship between the spatial coherence of each 
723 interferogram and its spatial and temporal baseline (Hooper et al., 2007; Zebker and Villasenor,

724 1992); while the second approach uses the observed spatial coherence on the manually specified

725 area of interest (blue rectangle in Fig. 10b and e). This approach more reliably identifies the

726 coherent interferograms, especially when the simple decorrelation model does not apply, e.g.

727 vegetated areas, long temporal baseline interferograms on Sierra Negra caldera with low

728 coherence due to high deformation phase gradient (Baran et al., 2005). The improvement in

729 temporal coherence using the second approach leads to additional reliable pixels (Fig. 10c and f).

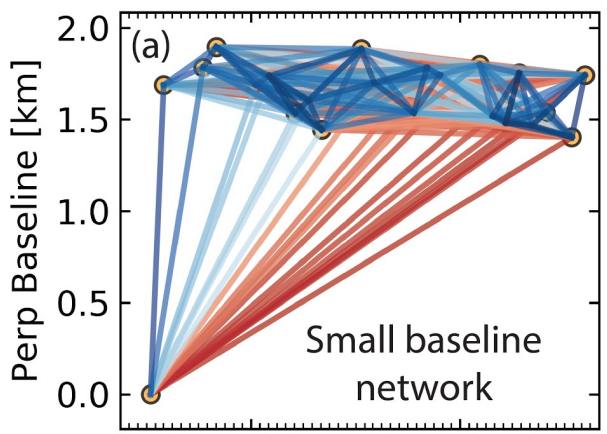

temporal coherence
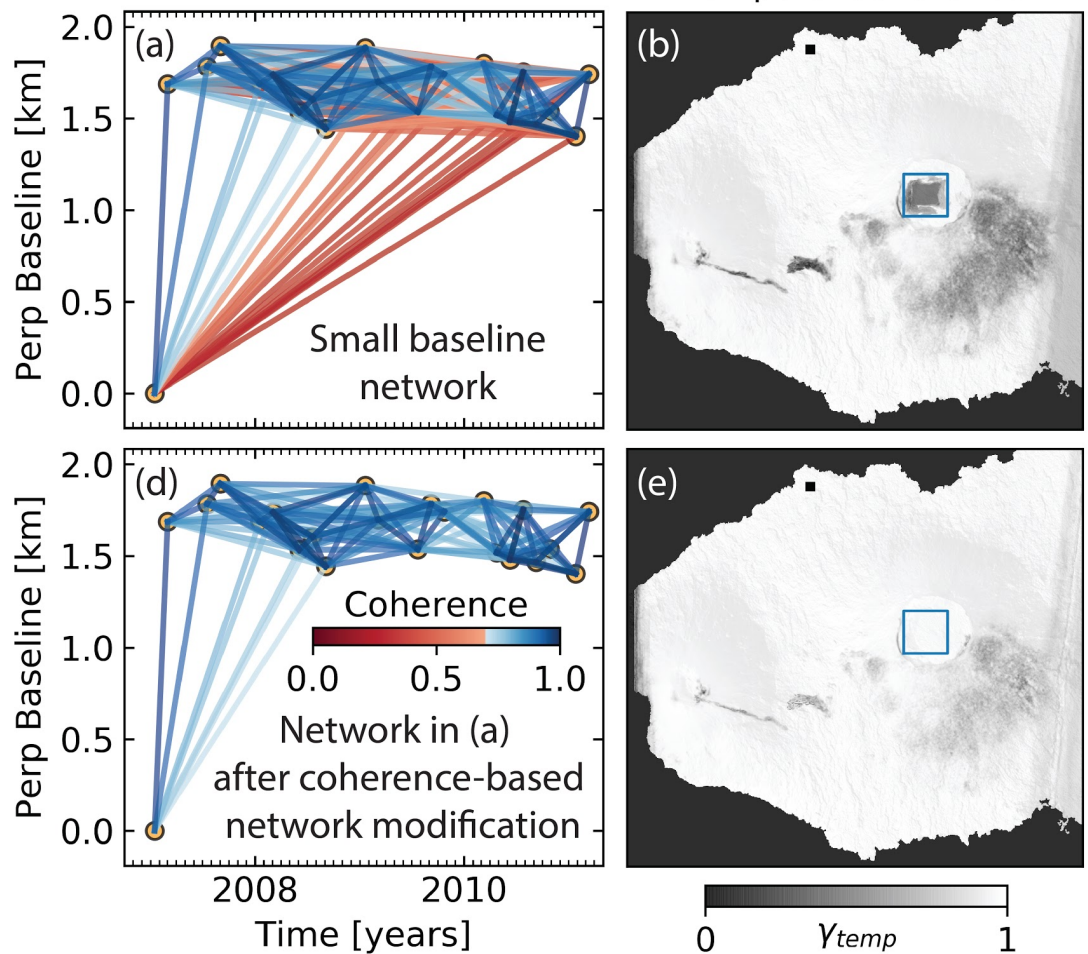

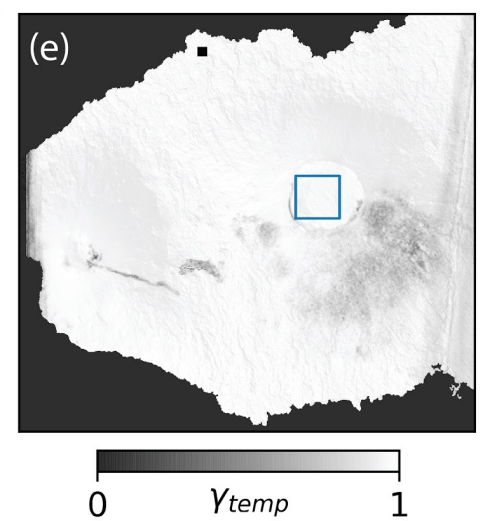

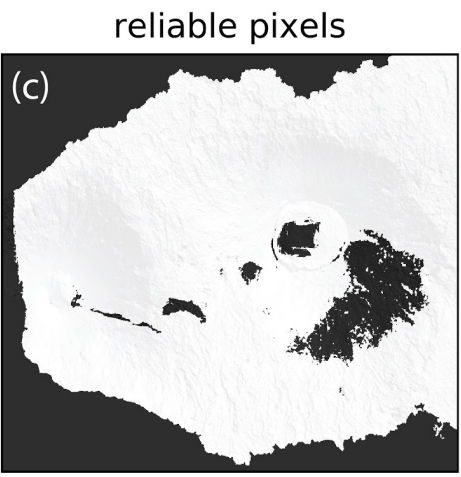

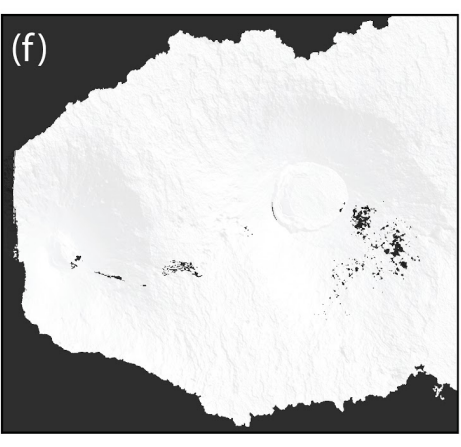

Figure 10. Impact of network modification on temporal coherence for ALOS-1 dataset. (a)

732 Network configuration, (b) temporal coherence and (c) reliable pixels with temporal coherence

$733 \geq 0.7$ from inversion of small baseline network with uniform weighting. (d-f): same as (a-c) but

734 from inversion of a network obtained by coherence-based network modification with inverse-

735 variance weighting. Lines in (a) and (d) represent interferograms colored by the average spatial 
A post print of a published manuscript at Computers and Geosciences

736 coherence within the Sierra Negra caldera (blue rectangles in (b and e)). Black squares in (b

737 and e) indicate the reference point.

\section{$738 \quad 5.3 .2$ Inverted raw phase}

739 The temporal filtering performed by the inversion of a redundant network of interferograms is

740 illustrated by comparing an observed interferogram with the interferogram reconstructed from

741 the inverted raw phase time-series (referred to by some authors as linked phase). Fig. 11 shows

742 an ALOS-1 interferogram with 3.5 years temporal baseline. The observed and the reconstructed

743 interferograms (Fig. 11a and b) are very similar except at the south and east of the caldera, where

744 the observed interferogram is incoherent but not the reconstructed interferogram as shown by the

745 high-frequency noise in the interferogram difference (Fig. 11c). This area is forested and

746 characterized by a low spatial coherence (Fig. 11d and e). This example, although with an

747 extreme temporal baseline, demonstrates how the network inversion filters out the temporal

748 decorrelation noise (Ansari, 2017; Guarnieri and Tebaldini, 2008; Pepe et al., 2015).

750 There is a difference in the north of the decorrelated area (yellow colors marked by white 751 rectangle in Fig. 11c). These areas are lightly vegetated (Fig. 11e), the discrepancy in phase is

752 likely caused by the soil or tree moisture considering its sensitivity to L-band SAR data (De Zan 753 and Gomba, 2018) and land cover (Fig. 11e). 
A post print of a published manuscript at Computers and Geosciences

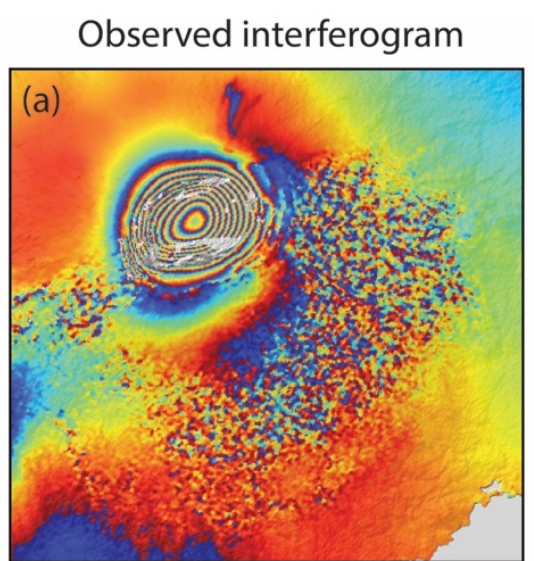

Spatial coherence of (a)

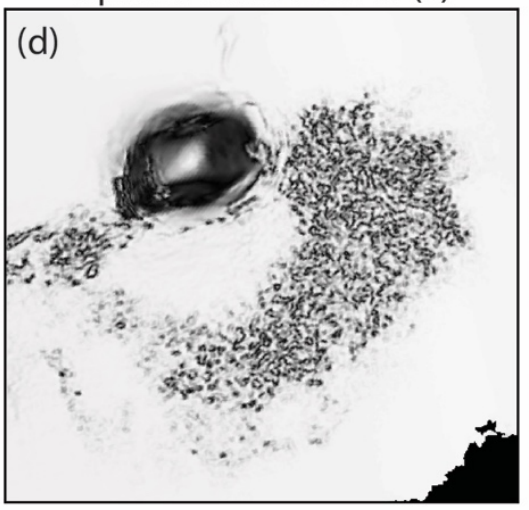

Reconstructed interferogram

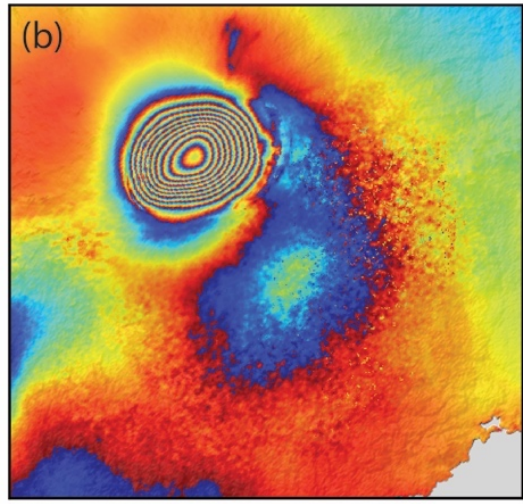

Google Earth image

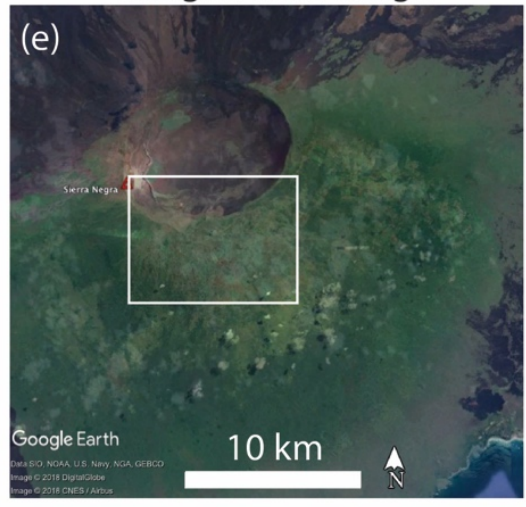

Interferogram difference
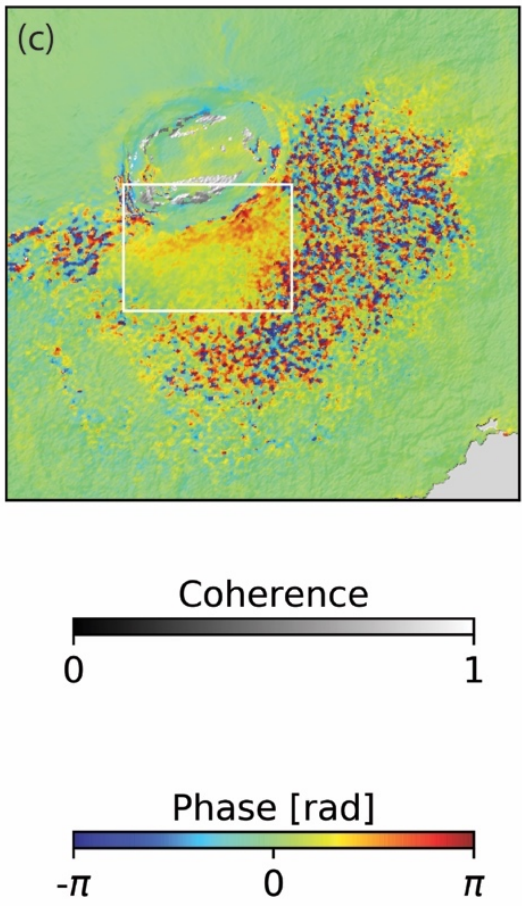

Figure 11. Spatial inspection of the inverted raw phase. (a) Observed interferometric phase and

756 (b) reconstructed phase from the inverted raw phase time-series; (c) difference between (a) and 757 (b); (d) observed spatial coherence; (e) optical image from Google Earth. The ALOS-1 758 interferogram has temporal baseline of 3.5 years (2 March 2007 - 10 September 2010) and 759 perpendicular baseline of $219 \mathrm{~m}$. In (a) part of the caldera is masked out during phase 760 unwrapping because of low coherence. White rectangles in (c and e): areas likely affected by soil 761 or tree moisture. The phase is wrapped into $[-\pi, \pi)$ for display.

\subsection{Noisy SAR acquisitions}

763 Noisy acquisitions with severe atmospheric delays or decorrelation noise could potentially bias

764 the estimation of topographic residuals, the average velocity or coefficients of any temporal 
765 deformation model. In the routine workflow, they are automatically identified and excluded in 766 the estimations.

768 Fig. 12 shows the impact of noisy acquisitions on the average velocity estimation for the L-band

769 ALOS-1 dataset. Several acquisitions are severely contaminated by ionospheric streaks and

770 identified by high residual phase RMS value (gray bars in Fig. 12a). Comparing the estimated

771 average velocities from displacement time-series with noisy acquisitions (Fig. 12b) and without

772 noisy acquisitions (Fig. 12c) reveals that excluding the noisy acquisitions significantly reduces

773 the estimation bias. The residual phase time-series $\hat{\phi}_{\text {resid }}$ estimated from equation (13) is shown 774 in supp. Fig. S7.
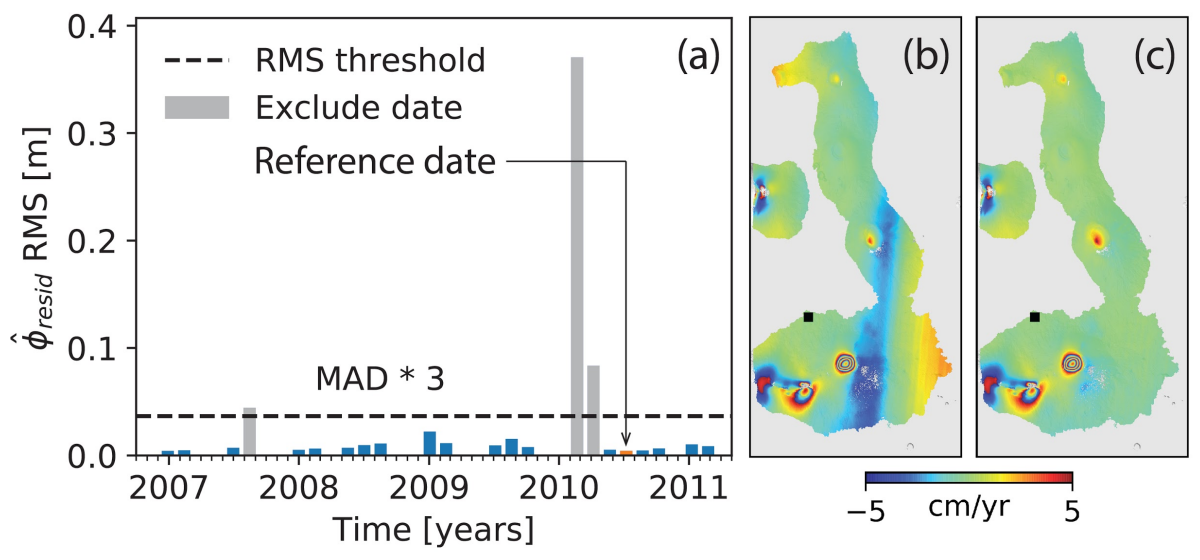

777 Figure 12. Impact of noisy acquisitions on velocity estimation. (a) RMS of the residual phase

778 estimates $\hat{\phi}_{\text {resid }}$ for each acquisition in the ALOS-1 dataset calculated using equation (14).

779 Dashed line: threshold (three times MAD of the RMS time-series by default). Gray bars: noisy 780 acquisitions with RMS larger than the threshold. (b and c): estimated average LOS velocities

781 from displacement time-series with and without noisy acquisitions, respectively. Velocities are 782 wrapped into $[-5,5) \mathrm{cm} / \mathrm{yr}$ for display. 
A post print of a published manuscript at Computers and Geosciences

\section{6. Discussion}

\section{$784 \quad 6.1$ Phase corrections in the time-series domain}

785 In the presented approach the phase corrections are applied in the time-series domain in contrast

786 to other approaches where they are applied in the interferogram domain (Agram et al., 2013;

787 Berardino et al., 2002). Both types of approaches give identical results, but the time-series

788 domain approach has two advantages: first, it is computationally more efficient because it uses

$789 N-1$ unwrapped phases, in contrast to the much larger number of interferograms for the

790 interferogram domain approach (up to $N \times(N-1) / 2$ for all possible interferograms); second,

791 the impact of the corrections is readily evaluated in both the spatial and temporal domains.

792

793 Fig. 13 upper panel (a) shows how the displacement at one acquisition is obtained by subtracting

794 the estimations of the tropospheric delay, of the phase ramp and of the topographic residual from

795 the raw phase. The time-series for a pixel along the southern coast of Isabela demonstrates the

796 power of the corrections (Fig. 13b). The area experienced a sill intrusion in March 2017 (dashed

797 line in Fig. 13b; Bagnardi and Hooper, 2018). The permanent ground displacement of $5 \mathrm{~cm}$ in

798 LOS direction is difficult to discern in the raw phase time-series but becomes visible after 799 applying the three corrections. Note that this pixel is far away from the intrusion in the first stage

800 and only affected by the intrusion in the second stage, thus showing only one jump in the

801 displacement time-series. For Sentinel-1 the topographic residuals are small (less than $4 \mathrm{~cm}$ in 802 this dataset) due to the small orbital tube but this is different for other sensors (Fattahi and 803 Amelung, 2013). 


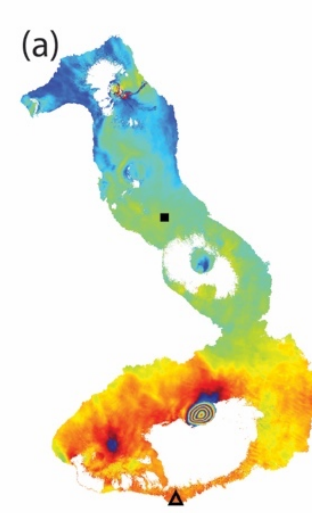

Raw phase

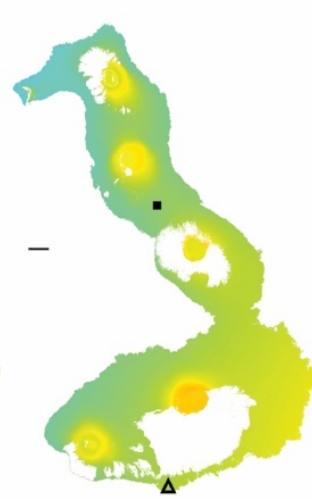

Tropospheric delay

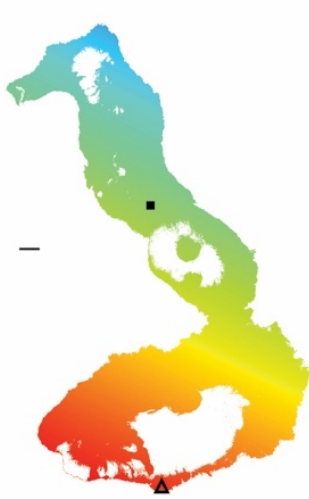

Phase ramp

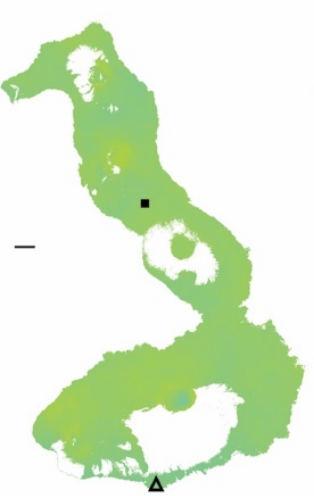

Topographic residual

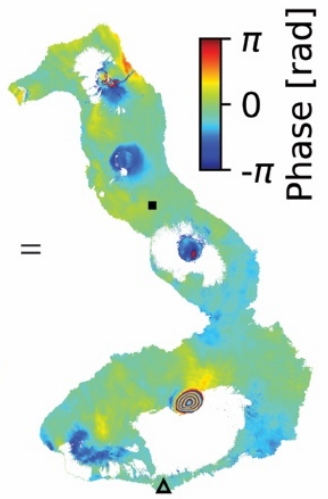

Displacement

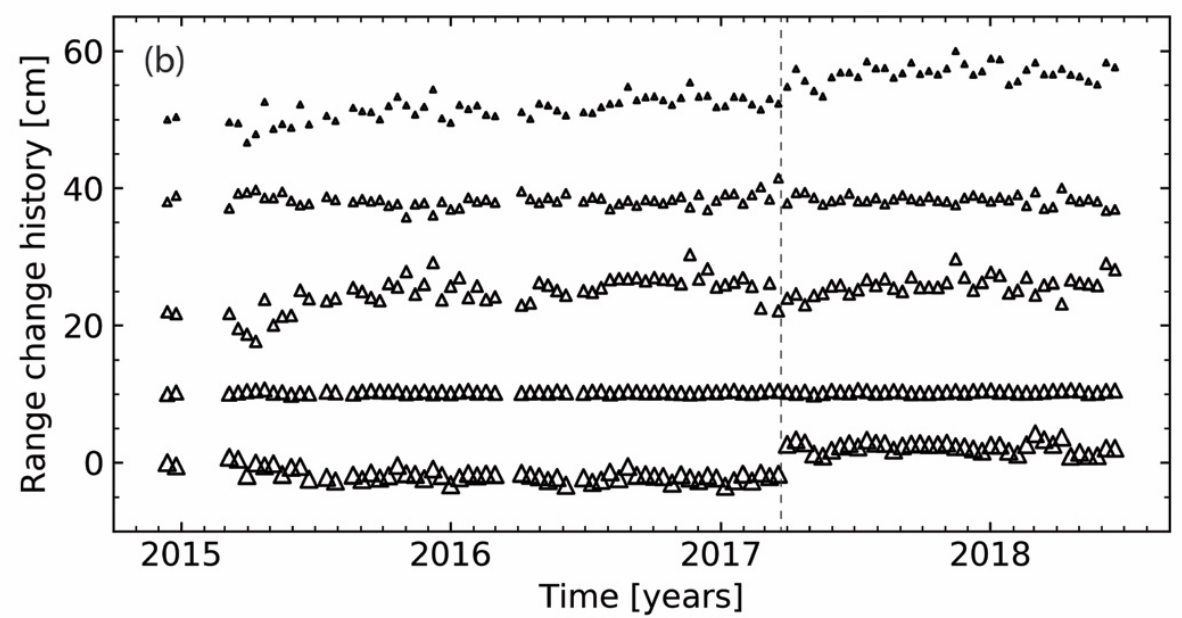

Raw phase

Tropospheric delay

Phase ramp

Topographic residual

Displacement

805 Figure 13. Illustration of phase corrections in the time-series domain: (a) at one acquisition (12

806 May 2016; the reference date is 27 September 2015); (b) at one pixel (southern flank of Cerro

807 Azul, marked as a triangle in the upper panel; [W91.1917, S1.0352 $])$. Displacements are

808 obtained by subtracting the estimated tropospheric delay, phase ramp and topographic residual

809 from the raw phase (equation (4)). Black squares in (a) indicate the reference point. Data are

810 wrapped into $[-\pi, \pi)$ for display. All range change histories in (b) start at zero but are shifted

811 for display. The permanent displacement due to a sill intrusion in March 2017 (marked as

812 dashed line) is visible after phase corrections. 
A post print of a published manuscript at Computers and Geosciences

\section{$813 \quad 6.2$ Order of phase corrections}

814 In our proposed workflow the tropospheric delay correction using external independent GAMs

815 should be applied first. The order of the other phase corrections is interchangeable because they

816 exploit different aspects of the InSAR data. Empirical tropospheric delay correction based on

817 delay-elevation ratio removes signals correlated with the topography. Phase deramping removes

818 signals correlated with the spatial coordinates (linearly or quadratically). Topographic residual

819 correction removes signals correlated in time with the perpendicular baseline. We recommend

820 applying phase deramping before topographic residual correction so that the estimated step

821 functions do not have to be deramped again.

\section{$822 \quad 6.3$ Interferogram network redundancy}

823 We consider stacks of Sentinel-1 interferograms from section 5 with different numbers of

824 sequential connections for each acquisition to assess the impact of network redundancy on the

825 estimation of (i) the displacement time-series and (ii) the temporal coherence (the reliability

826 measure). We compute the RMSE of the InSAR time-series at the GPS stations within Sierra

827 Negra caldera, assuming that the GPS measurements are the truth (see section 5.1; Fig. 14) and

828 examine the temporal coherence for these pixels. We also count the number of reliable pixels

829 (spatial coverage; temporal coherence $\geq 0.7$ ).

831 The average RMSE (bars in Fig. 14; GV10 excluded) decreases (improves) with the increasing 832 number of sequential connections rapidly until 5 connections then slowly until the reduction 833 becomes negligible. The temporal coherence (orange triangles in Fig. 14) stays at high values

834 (above 0.9) for all stations, except for GV10, for which it decreases to 0.65 at 4 connections and 835 to 0.24 at 20 connections. The low temporal coherence indicates that this is not a reliable pixel. It 
836 also has a relatively large RMSE (Fig. $8 \mathrm{~b}$ in section 5.1). This example shows that increasing

837 network redundancy leads to improved identification of reliable pixels. For this specific dataset,

838 a network of interferograms with 5 connections gives a good balance among precision, reliability

839 and spatial coverage (green dots in Fig. 14).

840

841 We note that in this case decorrelation noise is the dominant error source. Unwrapping errors 842 remaining after unwrapping error correction were excluded by removal of affected

843 interferograms using coherence-based network modification (see supp. Fig. S8). Still remaining 844 unwrap errors were suppressed by the weighting. Thus, more observations always help to reduce

845 the stochastic decorrelation noise, resulting in a more accurate estimation of the displacement 846 measurement (lower RMSE) and of the reliability measure (temporal coherence).

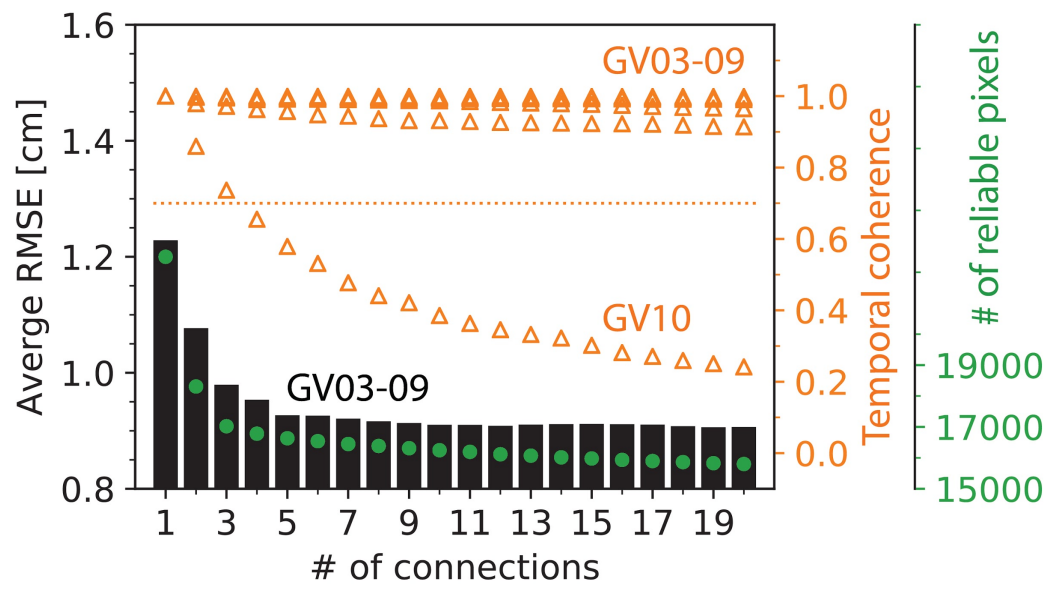

848 Figure 14. Average RMSE of InSAR time-series (black bars), temporal coherence (orange

849 triangles) at GPS stations and number of reliable pixels (green dots) as functions of the number 850 of sequential connections. Dotted orange line: temporal coherent threshold of 0.7.

852 As a practical implication, more interferograms are always preferred if the computing capacity 853 allows (Ansari et al., 2017). Since we cannot get the estimated spatial coherence before the 


\section{A post print of a published manuscript at Computers and Geosciences}

854 interferogram generation (due to the imperfect coherence model), generating a more redundant

855 network provides room to exclude low coherent interferograms especially those containing

856 reliable regions with unwrapping errors and still keep the network redundancy (temporal

857 coherence would always be one and meaningless if the system of network inversion is not

858 overdetermined, shown as orange triangles in Fig. 14 at 1 connection). In addition, a more

859 redundant network could potentially lead to a better unwrapping error correction based on phase

860 closure. Thus, we recommend using relatively relaxed interferogram selection thresholds (more

861 connections in sequential networks, larger temporal and perpendicular baselines in small baseline

862 networks) to generate more potentially coherent interferograms.

\section{$863 \quad 6.4$ Temporal coherence as the reliability measure}

864 We discuss the advantages and limitations of using the temporal coherence as the reliability

865 measure. An advantage is that the temporal coherence is a more robust reliability measure for the

866 inverted raw phase time-series compared to the average spatial coherence, because the temporal

867 coherence indicates not only the overall decorrelation noise, but also the overall level of non-

868 closing interferogram triplets. Non-closing triplets may be caused by the interferometric phase

869 residual (equation (1)), including decorrelation noise, possible phase-unwrapping errors and

870 interferometric phase contributions due to changes in the scatterers. An example of the latter is

871 the interferometric phase caused by changes in the dielectric properties of subsurface scatterers

872 in the result of soil moisture changes (De Zan et al., 2014; Morrison et al., 2011). Fig. 15a shows

873 how the temporal coherence is affected by unwrapping errors. In the absence of unwrapping

874 errors (pixels on Isabela island) the temporal and average spatial coherence are correlated but not

875 when unwrapping errors are present (pixels on Fernandina and Santiago islands). The

876 improvement in temporal coherence by phase-unwrapping error correction is illustrated in Fig. 9. 
878 However, a limitation is that the temporal coherence cannot capture temporal variations of the 879 reliability of the phase time-series. Fig. $15 \mathrm{~b}$ and $\mathrm{c}$ show the displacement time-series and 880 coherence matrix of a pixel that was covered by a lava flow during the 2015 Wolf eruption

881 (marked as a black triangle in Fig. 6). The surface change brings down the spatial coherence to 8820.3 during May-July 2015 (red grids in Fig. 15c), resulting in coherent, connected interferogram 883 networks only before and after the lava flow emplacement. This, however, has negligible impact 884 on the temporal coherence. With a temporal coherence of 0.94 the pixel is considered reliable 885 although valid displacement measurements were possible only before and after the flow 886 emplacement (after flow emplacement the pixel shows surface subsidence due to lava cooling).

887 A three-dimensional reliability measure such as the covariance matrix of decorrelation noise 888 (Agram and Simons, 2015) is more meaningful in this case of partially coherent scatterers, but 889 this is beyond the scope of this manuscript.
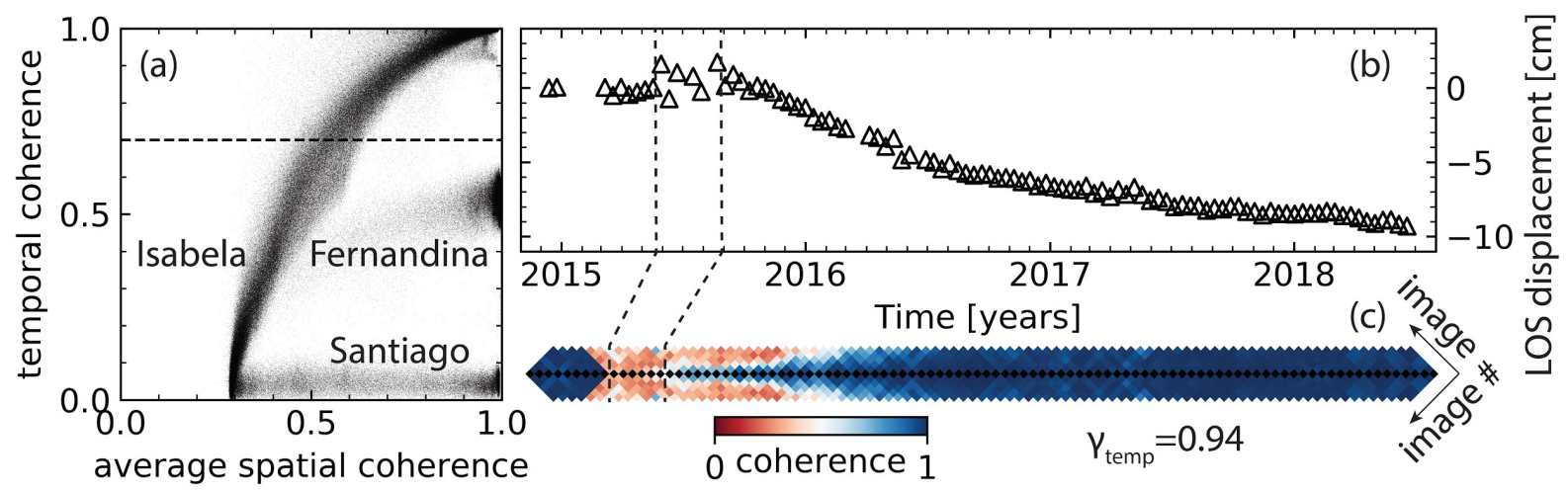

891 Figure 15. Advantage and limitation of temporal coherence as reliability measure. (a) Temporal

892 coherence versus average spatial coherence for land pixels of the Sentinel-1 dataset without 893 unwrapping error correction. Dashed line: default temporal coherence threshold of 0.7. Three 894 point clouds represent pixels on Isabela, Fernandina and Santiago islands. (b and c) 
A post print of a published manuscript at Computers and Geosciences

895 Displacement time-series and the diagonal section of coherence matrix of a pixel on the lava 896 flow of the 2015 Wolf eruption located at [W91.2838 , N0.0232 $]$ (black triangle in Fig. 6).

897 Reference pixel is located $\sim 600 \mathrm{~m}$ to the west $\left[W 91.2891^{\circ}, \mathrm{N0.0243^{ \circ }}\right]$. The coherence matrix is 898 rotated $45^{\circ}$ anticlockwise and shows the five diagonals below and above the main diagonal. 899 Dashed lines: period of lava flow emplacement.

\section{$900 \quad 6.5$ Comparing MintPy with GIAnT}

901 We compare the performance of the MintPy routine workflow with the classic SBAS approach

902 (Berardino et al, 2002), the New Small Baseline Subset (NSBAS) approach (Doin et al., 2011;

903 López-Quiroz et al., 2009) and the Multiscale InSAR Time-Series approach (Hetland et al., 904 2012), as implemented in the Generic InSAR Analysis Toolbox (GIAnT) (Agram et al., 2013) 905 and referred to as G-SBAS, G-NSBAS, and G-TimeFun, respectively. We use the Galápagos 906 Sentinel-1 dataset and a spatial coherence threshold of 0.25 (as commonly done with GIAnT, 907 Agram and Simons, 2015) for all approaches including MintPy. Tropospheric delays are 908 corrected from the ERA-Interim model using the PyAPS software (Jolivet et al., 2011).

910 In the following we discuss the differences between the four approaches (summarized in table 1).

911 We demonstrate the impact on the displacement time-series using three pixels (Fig. 16i): a high

912 coherent pixel (pixel A), a low coherent pixel (pixel B) and a high coherent pixel with 913 unwrapping errors and complex displacement (pixel C). The coherence matrices of the three 914 pixels are shown in Fig. 16j. For the high coherent pixel A, all approaches give nearly identical 915 results (Fig. 16i). 
A post print of a published manuscript at Computers and Geosciences

916

917

918

919

920

921

922

923

924

925

926

927

928

929

930

931

932

933

934 NSBAS give estimation results.

\subsubsection{Initial pixel selection}

\subsubsection{Weighted network inversion}

MintPy selects pixels which have for every SAR acquisition a minimum number of coherent interferograms (1 by default); G-SBAS and G-TimeFun select pixels that are coherent in all interferograms; while G-NSBAS selects pixels with a predefined total minimum number of coherent interferograms (we use a minimum of 300 out of 475). This leads to differences in the spatial measurement coverage between the four approaches (Fig. 16e-h). Compared with GSBAS and G-TimeFun, MintPy has better coverage within the calderas of Alcedo and Fernandina and along Alcedo's flank. G-NSBAS has the best spatial coverage among all approaches. The spatial coverages are shown by the distribution of the number of interferograms for pixels selected by the four approaches (Fig. 16a-d).

MintPy uses weighting (the inverse-variance by default) during the network inversion while the other three approaches in GIAnT do not. The impact on the estimated displacement time-series is not negligible when there is significant quality variation among the observations. One example is the displacement time-series of the low coherent pixel B in Fig. 16i. This is confirmed by the nearly identical result between G-NSBAS and MintPy without weighting (see supp. Fig. S9a).

Note that the asymmetric red grids along the horizontal black grids in Fig. 16j indicate the masked out interferogram due to spatial coherence thresholding, thus, only MintPy and GNSBAS give estimation results.

\subsubsection{Unwrapping error correction}

936 MintPy supports bridging and phase closure methods to correct unwrapping errors in the

937 interferograms, which GIAnT does not. Unwrap errors introduce bias in the estimated phase

938 ramps and displacement time-series. One example is the difference of the displacement time- 
A post print of a published manuscript at Computers and Geosciences

939 series on pixel C in Fig. 16i between MintPy and G-(N)SBAS. This is confirmed by the nearly

940 identical result between G-(N)SBAS and MintPy without unwrapping error correction (see supp.

941 Fig. S9b). The bias introduced by unwrapping errors is also evident in the velocity field at the

942 west side of Fernandina volcano (Fig. 16e-h).

\section{$943 \quad$ 6.5.4 No deformation model}

944 MintPy and G-SBAS do not assume temporal deformation model in network inversion. G-

945 NSBAS and G-TimeFun require temporal deformation models: G-NSBAS uses the model only

946 when the network is not fully connected in order to link multiple subsets of interferograms; while

947 G-TimeFun requires over-complete, potentially redundant models, which can be added manually

948 by user (Agram et al., 2013; Hetland et al., 2012). Thus, with the default configuration in this

949 case, G-TimeFun did not resolve the displacement jump due to the September 2017 Fernandina

950 eruption (pixel C in Fig. 16i).

\section{$951 \quad 6.5 .5$ Reliable pixel selection}

952 In contrast to approaches in GIAnT, MintPy assesses the quality of the inverted phase time-series

953 using temporal coherence and masks out unreliable pixels (gray area in Fig. 16a). We note that a

954 higher temporal coherence threshold ( 0.8 instead of the default 0.7$)$ is used because the spatial

955 coherence thresholding reduces the number of interferograms for unreliable pixels, bringing up

956 the temporal coherence value.

958 Table 1. Summary of the differences of time series analysis approaches in MintPy and GIAnT.

959 All approaches use small baseline network of unwrapped interferograms and linear optimization

960 time-series estimator. 
A post print of a published manuscript at Computers and Geosciences

\begin{tabular}{|c|c|c|c|c|}
\hline Aspect & MintPy & G-SBAS & G-NSBAS & G-TimeFun \\
\hline $\begin{array}{l}\text { initial pixel } \\
\text { selection }\end{array}$ & $\begin{array}{c}\text { a minimum } \\
\text { number of } \\
\text { coherent } \\
\text { interferograms } \\
\text { for every } \\
\text { acquisition }\end{array}$ & $\begin{array}{l}\text { coherent in all } \\
\text { interferogram } \\
\mathrm{s}\end{array}$ & $\begin{array}{c}\text { a total } \\
\text { minimum } \\
\text { number of } \\
\text { coherent } \\
\text { interferograms }\end{array}$ & $\begin{array}{l}\text { coherent in all } \\
\text { interferograms }\end{array}$ \\
\hline weighted inversion & yes & no & no & no \\
\hline $\begin{array}{l}\text { unwrapping error } \\
\text { correction }\end{array}$ & $\begin{array}{c}\text { bridging / } \\
\text { phase closure }\end{array}$ & no & no & no \\
\hline $\begin{array}{l}\text { posterior quality } \\
\text { assessment }\end{array}$ & yes & no & no & no \\
\hline $\begin{array}{c}\text { prior deformation } \\
\text { model }\end{array}$ & no & no & yes & yes \\
\hline $\begin{array}{c}\text { phase correction } \\
\text { operation }\end{array}$ & $\begin{array}{l}\text { time-series } \\
\text { domain }\end{array}$ & $\begin{array}{l}\text { interferogram } \\
\text { domain }\end{array}$ & $\begin{array}{c}\text { interferogram } \\
\text { domain }\end{array}$ & $\begin{array}{c}\text { interferogram } \\
\text { domain }\end{array}$ \\
\hline
\end{tabular}


A post print of a published manuscript at Computers and Geosciences
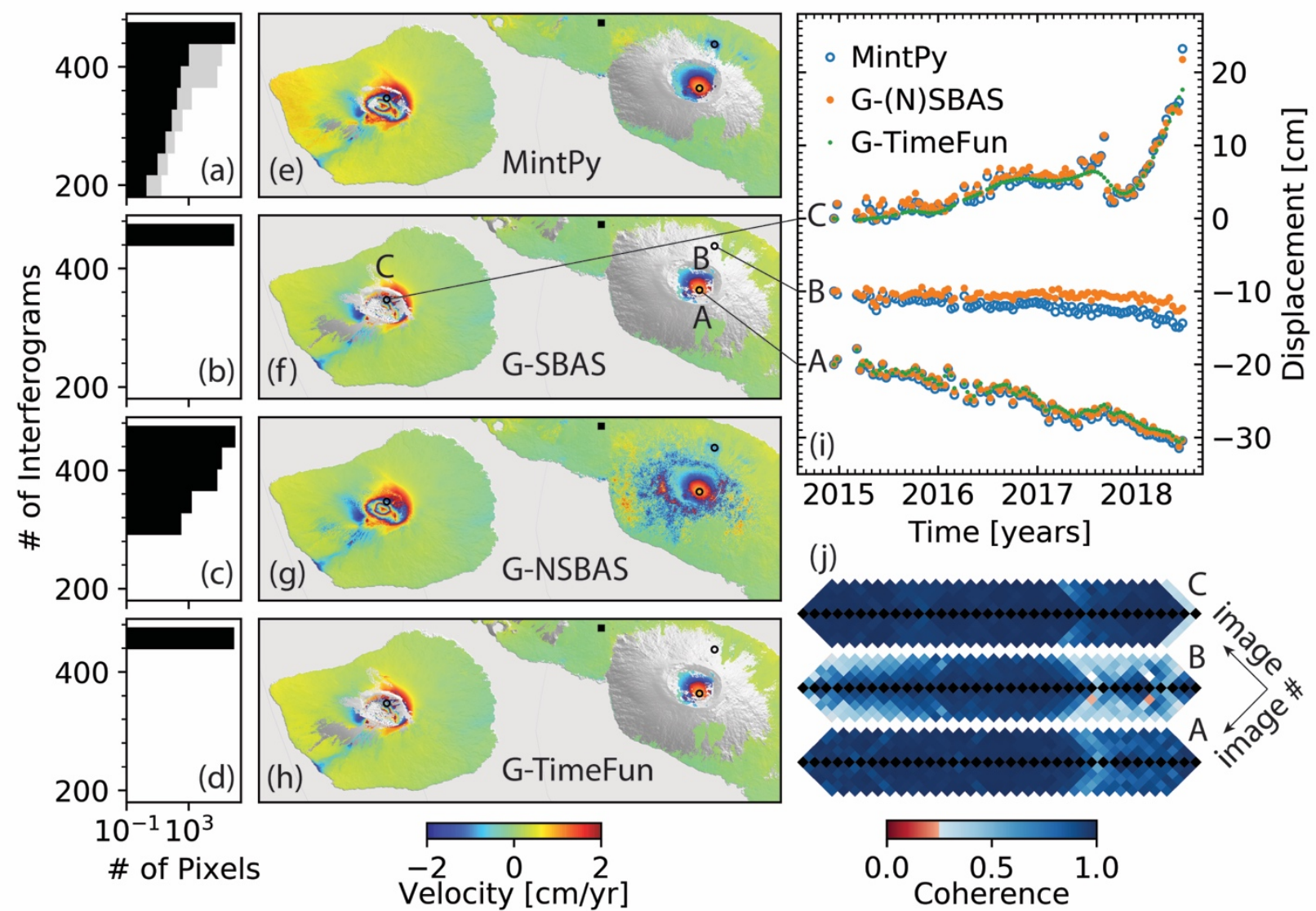

962

Figure 16. Comparison of MintPy with GIAnT approaches for the Sentinel-1 dataset for the

964 Galápagos. (a-d) Distribution of the number of interferograms for pixels used (number of pixels

965 for each interferogram bin) by the four time-series approaches on the entire Isabela and

966 Fernandina islands in log scale. Gray area in (a): unreliable pixels (pixels processed but

967 discarded because of low temporal coherence). (e-h) LOS velocity estimated from the

968 displacement time-series produced by the four time series approaches on Fernandina and Alcedo

969 volcano. Velocities are wrapped into [-2, 2) cm/yr for display. Black squares: reference point.

970 (i) Displacement time-series for pixels marked in (e-h). (j) Coherence matrix for pixels in (i)

971 (rotated to make the matrix diagonal line horizontal; only showed the main diagonal and the five

972 diagonals below and above; only showed the data from 7 May 2017 - 19 June 2018). The lower

973 and upper half: interferograms before and after phase masking, respectively. The asymmetric 
A post print of a published manuscript at Computers and Geosciences

974 red grids between the upper and lower half for pixel B indicate masked out interferograms with

975 spatial coherence $<0.25$.

\section{7. Summary and conclusions}

977 We have reviewed the mathematical formulation for the weighted network inversion and for the 978 post-inversion phase corrections for time series analysis of small baseline InSAR stacks. In 979 contrast to some persistent scatterer methods, the presented approach does not require prior 980 deformation models or temporal filtering and is therefore well suited to extract nonlinear 981 displacements. Reliable pixels are identified using the temporal coherence. Noisy acquisitions

982 with severe atmospheric turbulence are identified using an outlier detection method based on the 983 median absolute deviation of the residual phase RMS and are excluded during the estimations of 984 topographic residual and average velocity.

985

986 Our workflow includes two methods to correct for, and one method to exclude remaining phase987 unwrapping errors. The first unwrapping error correction method is bridging. This method uses 988 MST bridges to connect the reliable regions of each interferogram, assuming that the phase 989 differences between neighboring regions are less than $\pi \mathrm{rad}$ in magnitude. This method is 990 particularly well-suited for islands and/or areas with steep topography. The second method is the 991 phase closure method. This method exploits the conservativeness of the integer ambiguities of 992 interferogram triplets. A sparse solution for the phase-unwrapping integer ambiguity is obtained 993 using the $L^{1}$-norm regularized least squares approximation. Coherent phase-unwrapping errors 994 can be identified using the distribution of the number of triplets with non-zero integer ambiguity 995 of the closure phase. Best results are obtained by combining these two methods. 


\section{A post print of a published manuscript at Computers and Geosciences}

997 The method to exclude remaining coherent phase-unwrapping errors is coherence-based network

998 modification. In this approach affected interferograms are identified and excluded using a

999 threshold of average spatial coherence calculated over a customized area of interest that includes

1000 the low coherent areas surrounding the areas with coherent phase-unwrapping errors.

1002 We have applied the routine workflow to ALOS-1 and Sentinel-1 data acquired over the 1003 Galápagos volcanoes. The InSAR results show very good agreement with independent GPS 1004 measurements. A comparison with the algorithms implemented in the GIAnT software shows

1005 similar performance in the high coherent areas but superior performance in the low coherent 1006 areas and the high coherent areas with phase-unwrapping errors or complex displacement 1007 because of unwrapping error correction, weighted network inversion, initial and reliable pixel 1008 selection using temporal coherence.

1010 We investigated how some configurations of the routine workflow affect the precision and 1011 accuracy of the InSAR measurement using real and/or simulated data. The conclusions are:

1013 1. Inverse-variance weighting gives the most robust and one of the best performances for 1014 network inversion among four different weighting functions: uniform, coherence, 1015 inverse-variance and Fisher information matrix.

10162 . For interferogram networks with 3, 5 and 10 sequential connections, the phase closure 1017 method fully corrects for phase-unwrapping errors if less than 5, 20 and $35 \%$ of the interferograms are affected by phase-unwrapping errors, respectively (with maximum 
errors of 2 cycles). This shows that the phase closure method performs better for more redundant networks.

3. Increasing the network redundancy improves the network inversion and the estimation of temporal coherence (as long as phase-unwrapping errors have been corrected or excluded), resulting in more accurate estimation of the displacement time-series and identification of reliable pixels. Thus, we recommend using more connections in sequential networks, and to use larger temporal and perpendicular baselines in small baseline networks.

4. The order of the InSAR-data-dependent phase corrections (the empirical tropospheric delay correction based on the delay-elevation ratio, topographic residual correction and phase deramping) is interchangeable and has negligible impact on the noise-reduced displacement time-series.

5. Temporal coherence is a more robust reliability measure than average spatial coherence because it accounts for phase-unwrapping errors. However, it does not capture temporal variations of the reliability of the phase time-series, limiting its usefulness for partially coherent scatterers.

\section{Author contribution}

$1036 \mathrm{HF}$ and ZY developed the mathematical scope. ZY and HF developed the software. ZY and FA

1037 tested the software and processed the data. ZY wrote the manuscript with the help of FA and HF. 1038 FA supervised the project. 
A post print of a published manuscript at Computers and Geosciences

1039

1040

1041 (MintPy), with open-source code, documentation, tutorials in Jupyter Notebook and test data

1042 freely available on GitHub (https://github.com/insarlab/MintPy) under GNU Generic Public

1043 License version 3. Figures in this manuscript are plotted using Jupyter Notebook and available 1044 on GitHub (https://github.com/geodesymiami/Yunjun_et al-2019-MintPy). Time-series products

1045 from the routine workflow in this manuscript are available at https://zenodo.org/record/3464191 1046 and displayed at https://insarmaps.miami.edu.

\section{Declaration of competing interest}

1048 The authors declare that they have no known competing financial interests or personal 1049 relationships that could have appeared to influence the work reported in this paper.

\section{Acknowledgments}

1051 The Sentinel-1 and ALOS-1 data were provided by ESA and JAXA, respectively, and obtained 1052 from Alaska Satellite Facility (ASF) via the Seamless SAR Archive (SSARA), a service 1053 provided by the UNAVCO facility. The ownership of ALOS-1 data belongs to JAXA and the 1054 Ministry of Economy, Trade and Industry. GPS data was provided by the University of Nevada, 1055 Reno. We thank Yunmeng Cao and Sara Mirzaee for discussions, Xiaohua Xu for pointing us to 1056 the sparse solution of the integer ambiguity of the closure phase. We thank undergraduate 1057 students Joshua Zahner, David Grossman and Alfredo Terrero for code contributions. The 1058 software is based on the initial code by Noel Gourmelen and Scott Baker. This work was 1059 supported by NASA Headquarters under the Earth and Space Science Fellowship program 
1060 (Grant No. NNX15AN13H), the NISAR Science Team (Grant No. NNX16AK52G) and National 1061 Science Foundation's Geophysics program (Grant No. EAR1345129). Part of the research was 1062 carried out at the Jet Propulsion Laboratory, California Institute of Technology, under a contract 1063 with the National Aeronautics and Space Administration.

1064 Appendix A. Supplementary data

1065 Supplementary data to this article can be found online at

1066 https://doi.org/10.1016/j.cageo.2019.104331.

1067 Appendix B. List of acronyms and symbols

1068 Table B1. List of acronyms

1069 DS Distributed scatterer.

1070 FIM Fisher information matrix.

1071 GAM Global atmospheric model.

1072 GIAnT Generic InSAR Analysis Toolbox.

1073 G-SBAS Small baseline subset in GIAnT.

1074 G-NSBAS New small baseline subset in GIAnT.

1075 G-TimeFun Multiscale InSAR Time-Series in GIAnT.

1076 LASSO Least absolute shrinkage and selection operator.

1077 LOS Line of sight.

1078 MAD Median absolute deviation.

1079 MST Minimum spanning tree.

1080 PDF Probability density function. 


$\begin{array}{lll}1081 & \text { PS } & \text { Persistent scatterer. } \\ 1082 & \text { RMS } & \text { Root mean square. } \\ 1083 & \text { RMSE } & \text { Root mean square error. } \\ 1084 & \text { SBAS } & \text { Small baseline subset. } \\ 1085 & \text { SLC } & \text { Single look complex. } \\ 1086 & \text { SNAPHU } & \text { Statistical-cost, Network-flow Algorithm for Phase Unwrapping. } \\ 1087 & \text { WLS } & \text { Weighted least squares. }\end{array}$

1088 Table B2. List of symbols

1089 Symbol Parameter

$1091 \boldsymbol{A}$ Design matrix for network inversion in size of $M \times(N-1)$.

1092 C Design matrix for the closure phase of interferogram triplets.

$1093 \boldsymbol{H} \quad$ All-one column matrix in size of $M \times 1$.

$1094 L \quad$ Number of looks in range and azimuth directions in total.

$1095 M \quad$ Number of interferograms.

$1096 N \quad$ Number of SAR acquisitions.

$1097 T \quad$ Number of interferogram triplets.

$1098 U \quad$ Matrix of the phase-unwrapping integer ambiguity in size of $M \times 1$.

$1099 \boldsymbol{W}$ Weight matrix for network inversion in size of $M \times M$.

$1100 C^{i j k} \quad$ Closure phase of the interferogram triplet formed from acquisitions at $t_{i}, t_{j}$, and $t_{k}$.

$1101 \quad C_{i n t}^{i j k} \quad$ Integer ambiguity of $C^{i j k}$.

$1102 \quad T_{\text {int }} \quad$ Number of triplets with non-zero $C_{i n t}^{i j k}$ among all triplets.

$1103 \Delta \phi^{j} \quad$ Interferometric phase of the $j_{t h}$ unwrapped interferogram. 
A post print of a published manuscript at Computers and Geosciences

$1104 \Delta \phi_{\varepsilon}^{j} \quad$ Interferometric phase residual of the $j_{\text {th }}$ unwrapped interferogram.

$1105 \Delta \phi \quad$ Vector of the interferometric phase of all interferograms.

$1106 \Delta \phi_{\varepsilon} \quad$ Vector of the interferometric phase residual of all interferograms.

$1107 \phi^{i} \quad$ Raw phase between the $i_{t h}$ and the $1_{s t}$ acquisition.

$1108 \phi \quad$ Vector of raw phase of all acquisitions (raw phase time-series).

$1109 \hat{\phi} \quad$ The estimated vector of raw phase time-series.

$1110 \quad \phi_{d i s}^{i} \quad$ Phase due to the displacement between the $i_{t h}$ and the $1_{s t}$ acquisition.

$1111 \hat{\phi}_{\text {tropo }}^{i} \quad$ Estimated tropospheric delay between the $i_{t h}$ and the $1_{s t}$ acquisition.

$1112 \hat{\phi}_{\text {geom }}^{i} \quad$ Estimated geometrical range difference between the $i_{\text {th }}$ and the $1_{s t}$ acquisition

1113 caused by the non-zero spatial baseline.

$1114 \phi_{\text {resid }}^{i} \quad$ Residual phase remained between the $i_{t h}$ and the $1_{s t}$ acquisition.

$1115 \phi_{\text {resid }} \quad$ Vector of the residual phase of all acquisitions (residual phase time-series)

$1116 \widehat{\phi}_{\text {resid }}(p) \quad$ Estimated vector of the residual phase time-series on pixel $p$.

$1117 \delta L_{p}^{i} \quad$ Integrated absolute single path tropospheric delay between the $i_{t h}$ and the $1_{s t}$

1118 acquisition on pixel $p$ in meters.

$1119 \hat{\phi}_{\text {tropo }}^{i}(p) \quad$ Estimated phase of the relative double path tropospheric delay between the $i_{\text {th }}$ and

1120 the $1_{s t}$ acquisition on pixel $p$ with respect to pixel ref.

$1121 \sigma_{\Delta \phi^{j}}^{2} \quad$ Variance of the interferometric phase of the $j_{t h}$ interferogram.

$1122 \gamma^{j} \quad$ Spatial coherence of $j_{\text {th }}$ interferogram.

$1123 \gamma_{\text {temp }} \quad$ Temporal coherence.

$1124 \lambda \quad$ Radar wavelength in meters.

$1125 \quad Z_{\varepsilon} \quad$ Topographic residual in meters. 


\section{A post print of a published manuscript at Computers and Geosciences}

\section{Reference}

1127 Agram, P. S., R. Jolivet, B. Riel, Y. N. Lin, M. Simons, E. Hetland, M. P. Doin, and C. Lasserre, 2013. New Radar

1128 Interferometric Time Series Analysis Toolbox Released, Eos, Transactions American Geophysical Union, 94(7), 1129 69-70, doi :10.1002/2013EO070001.

1130 Agram, P., and M. Simons, 2015. A noise model for InSAR time series, Journal of Geophysical Research: Solid $1131 \quad$ Earth, 120(4), 2752-2771, doi:10.1002/2014JB011271.

1132 Andersen, M., J. Dahl, Z. Liu, and L. Vandenberghe, 2011. Interior-point methods for large-scale cone 1133 programming, in Optimization for machine learning, edited by S. Sra, S. Nowozin and S. J. Wright, MIT Press.

1134 Ansari, H., F. D. Zan, and R. Bamler, 2017. Sequential Estimator: Toward Efficient InSAR Time Series Analysis, 1135 IEEE Transactions on Geoscience and Remote Sensing, 55(10), 5637-5652, doi:10.1109/TGRS.2017.2711037.

1136 Ansari, H., F. D. Zan, and R. Bamler, 2018. Efficient Phase Estimation for Interferogram Stacks, IEEE Transactions 1137 on Geoscience and Remote Sensing, 56(7), 4109-4125, doi:10.1109/TGRS.2018.2826045.

1138 Bagnardi, M., and A. Hooper, 2018. Inversion of Surface Deformation Data for Rapid Estimates of Source 1139 Parameters and Uncertainties: A Bayesian Approach, Geochemistry, Geophysics, Geosystems, 19, 1140 doi:10.1029/2018GC007585.

1141 Baran, I., M. Stewart, and S. Claessens, 2005. A new functional model for determining minimum and maximum 1142 detectable deformation gradient resolved by satellite radar interferometry, IEEE Transactions on Geoscience and 1143 Remote Sensing, 43(4), 675-682, doi:10.1109/TGRS.2004.843187.

1144 Bekaert, D. P. S., A. Hooper, and T. J. Wright, 2015. A spatially-variable power-law tropospheric correction 1145 technique for InSAR data, Journal of Geophysical Research: Solid Earth, 120(2), 1345-1356, 1146 doi:10.1002/2014JB011558.

1147 Berardino, P., G. Fornaro, R. Lanari, and E. Sansosti, 2002. A new algorithm for surface deformation monitoring 1148 based on small baseline differential SAR interferograms, Geoscience and Remote Sensing, IEEE Transactions 1149 on, 40(11), 2375-2383, doi:10.1109/TGRS.2002.803792.

1150 Biggs, J., T. Wright, Z. Lu, and B. Parsons, 2007. Multi-interferogram method for measuring interseismic 1151 deformation: Denali Fault, Alaska, Geophysical Journal International, 170(3), 1165-1179, doi:10.1111/j.1365$1152 \quad$ 246X.2007.03415.x. 


\section{A post print of a published manuscript at Computers and Geosciences}

1153 Blewitt, G., W.C. Hammond, C. Kreemer, 2018. Harnessing the GPS Data Explosion for Interdisciplinary Science, $1154 \quad$ Eos, 99, doi:10.1029/2018EO104623.

1155 Cao, N., H. Lee, and H. C. Jung, 2015. Mathematical Framework for Phase-Triangulation Algorithms in 1156 Distributed-Scatterer Interferometry, IEEE Geoscience and Remote Sensing Letters, 12(9), 1838-1842, 1157 doi:10.1109/LGRS.2015.2430752.

1158 Carballo, G. F., and P. W. Fieguth, 2002. Hierarchical network flow phase unwrapping, IEEE Transactions on 1159 Geoscience and Remote Sensing, 40(8), 1695-1708, doi:10.1109/TGRS.2002.800279.

1160 Chaussard, E., F. Amelung, and Y. Aoki, 2013. Characterization of open and closed volcanic systems in Indonesia 1161 and Mexico using InSAR time series, Journal of Geophysical Research: Solid Earth, 118(8), 3957-3969, 1162 doi:10.1002/jgrb.50288.

1163 Chaussard, E., R. Bürgmann, H. Fattahi, R. M. Nadeau, T. Taira, C. W. Johnson, and I. Johanson, 2015. Potential 1164 for larger earthquakes in the East San Francisco Bay Area due to the direct connection between the Hayward and 1165 Calaveras Faults, Geophysical Research Letters, 42(8), 2734-2741, doi:10.1002/2015GL063575.

1166 Chen, C. W., and H. A. Zebker, 2001. Two-dimensional phase unwrapping with use of statistical models for cost 1167 functions in nonlinear optimization, JOSA A, 18(2), 338-351, doi:10.1364/JOSAA.18.000338.

1168 Chen, C. W., and H. A. Zebker, 2002. Phase unwrapping for large SAR interferograms: statistical segmentation and 1169 generalized network models, Geoscience and Remote Sensing, IEEE Transactions on, 40(8), 1709-1719, 1170 doi:10.1109/TGRS.2002.802453.

1171 Cormen, T. H., C. E. Leiserson, R. L. Rivest, and C. Stein, 2009. Introduction to algorithms, MIT press. Chap. 22.2

1172 De Zan, F., A. Parizzi, P. Prats-Iraola, and P. López-Dekker, 2014. A SAR Interferometric Model for Soil Moisture, 1173 IEEE Transactions on Geoscience and Remote Sensing, 52(1), 418-425, doi:10.1109/TGRS.2013.2241069.

1174 De Zan, F., and G. Gomba, 2018. Vegetation and soil moisture inversion from SAR closure phases: First 1175 experiments and results, Remote Sensing of Environment, 217, 562-572, doi:10.1016/j.rse.2018.08.034.

1176 DiCaprio, C. J., and M. Simons, 2008. Importance of ocean tidal load corrections for differential InSAR, 1177 Geophysical Research Letters, 35(22), doi:10.1029/2008GL035806.

1178 Doin, M. P., C. Lasserre, G. Peltzer, O. Cavalié, and C. Doubre, 2009. Corrections of stratified tropospheric delays 1179 in SAR interferometry: Validation with global atmospheric models, Journal of Applied Geophysics, 69(1), 35-50, 1180 doi:10.1016/j.jappgeo.2009.03.010. 


\section{A post print of a published manuscript at Computers and Geosciences}

1181 Doin, M.-P., Lodge, F., Guillaso, S., Jolivet, R., Lasserre, C., Ducret, G., Grandin, R., Pathier, E., Pinel, V., 2011.

1182 In: Presentation of the small baseline NSBAS processing chain on a case example: the Etna deformation

1183 monitoring from 2003 to 2010 using Envisat data. Proceedings of the Fringe Symposium (SP-697), pp. 1-7.

1184 Farr, T. G., et al., 2007. The Shuttle Radar Topography Mission, Reviews of Geophysics, 45(2), 1185 doi:10.1029/2005RG000183.

1186 Fattahi, H., and F. Amelung, 2013. DEM Error Correction in InSAR Time Series, Geoscience and Remote Sensing, 1187 IEEE Transactions on, 51(7), 4249-4259, doi:10.1109/TGRS.2012.2227761.

1188 Fattahi, H., and F. Amelung, 2014. InSAR uncertainty due to orbital errors, Geophysical Journal International, 1189 199(1), 549-560, doi:10.1093/gji/ggu276.

1190 Fattahi, H., and F. Amelung, 2015. InSAR bias and uncertainty due to the systematic and stochastic tropospheric 1191 delay, Journal of Geophysical Research: Solid Earth, 120(12), 8758-8773, doi:10.1002/2015JB012419.

1192 Fattahi, H., P. Agram, and M. Simons, 2016. A Network-Based Enhanced Spectral Diversity Approach for TOPS 1193 Time-Series Analysis, IEEE Transactions on Geoscience and Remote Sensing, 55(2), 777-786, 1194 doi:10.1109/TGRS.2016.2614925.

1195 Fattahi, H., M. Simons, and P. Agram, 2017. InSAR Time-Series Estimation of the Ionospheric Phase Delay: An 1196 Extension of the Split Range-Spectrum Technique, IEEE Transactions on Geoscience and Remote Sensing, 1197 55(10), 5984-5996, doi:10.1109/TGRS.2017.2718566.

1198 Ferretti, A., C. Prati, and F. Rocca, 2001. Permanent scatterers in SAR interferometry, Geoscience and Remote 1199 Sensing, IEEE Transactions on, 39(1), 8-20, doi:10.1109/36.898661.

1200 Ferretti, A., A. Fumagalli, F. Novali, C. Prati, F. Rocca, and A. Rucci, 2011. A New Algorithm for Processing 1201 Interferometric Data-Stacks: SqueeSAR, Geoscience and Remote Sensing, IEEE Transactions on, 49(9), 3460$1202 \quad 3470$, doi:10.1109/tgrs.2011.2124465.

1203 Fornaro, G., Verde, S., Reale, D., Pauciullo, A., 2015. CAESAR: An Approach Based on Covariance Matrix 1204 Decomposition to Improve Multibaseline-Multitemporal Interferometric SAR Processing. Geoscience and 1205 Remote Sensing, IEEE Transactions on 53 (4), 2050-2065. doi:10.1109/TGRS.2014.2352853.

1206 Gomba, G., A. Parizzi, F. D. Zan, M. Eineder, and R. Bamler, 2016. Toward Operational Compensation of 1207 Ionospheric Effects in SAR Interferograms: The Split-Spectrum Method, IEEE Transactions on Geoscience and 1208 Remote Sensing, 54(3), 1446-1461, doi:10.1109/TGRS.2015.2481079. 


\section{A post print of a published manuscript at Computers and Geosciences}

1209 Guarnieri, A. M., and S. Tebaldini, 2007. Hybrid Cramér-Rao bounds for crustal displacement field estimators in 1210 SAR interferometry, Signal Processing Letters, IEEE, 14(12), 1012-1015, doi:10.1109/LSP.2007.904705.

1211 Guarnieri, A. M., and S. Tebaldini, 2008. On the exploitation of target statistics for SAR interferometry applications, 1212 Geoscience and Remote Sensing, IEEE Transactions on, 46(11), 3436-3443, doi:10.1109/TGRS.2008.2001756.

1213 Hanssen, R. F., 2001. Radar interferometry: data interpretation and error analysis, Kluwer Academic Pub, 1214 Dordrecht, Netherlands.

1215 Hetland, E., P. Musé, M. Simons, Y. Lin, P. Agram, and C. DiCaprio, 2012. Multiscale InSAR time series (MInTS) 1216 analysis of surface deformation, Journal of Geophysical Research: Solid Earth, 117(B2), 1217 doi:10.1029/2011JB008731.

1218 Hooper, A., H. Zebker, P. Segall, and B. Kampes, 2004. A new method for measuring deformation on volcanoes and 1219 other natural terrains using InSAR persistent scatterers, Geophysical Research Letters, 31(23), L23611, 1220 doi:10.1029/2004GL021737.

1221 Hooper, A., P. Segall, and H. Zebker, 2007. Persistent scatterer interferometric synthetic aperture radar for crustal 1222 deformation analysis, with application to Volcán Alcedo, Galápagos, Journal of Geophysical Research: Solid Earth, 112(B7), doi:10.1029/2006JB004763.

1224 Hussain, E., A. Hooper, T. J. Wright, R. J. Walters, and D. P. S. Bekaert, 2016. Interseismic strain accumulation across the central North Anatolian Fault from iteratively unwrapped InSAR measurements, Journal of Geophysical Research: Solid Earth, 121(12), 9000-9019, doi:10.1002/2016JB013108.

1227 Jolivet, R., R. Grandin, C. Lasserre, M. P. Doin, and G. Peltzer, 2011. Systematic InSAR tropospheric phase delay 1228 corrections from global meteorological reanalysis data, Geophysical Research Letters, 38(17), L17311, 1229 doi:10.1029/2011GL048757.

1230 Jolivet, R., P. S. Agram, N. Y. Lin, M. Simons, M. P. Doin, G. Peltzer, and Z. Li, 2014. Improving InSAR geodesy 1231 using global atmospheric models, Journal of Geophysical Research: Solid Earth, 119(3), 2324-2341, 1232 doi:10.1002/2013JB010588.

1233 Lanari, R., O. Mora, M. Manunta, J. J. Mallorquí, P. Berardino, and E. Sansosti (2004), A small-baseline approach 1234 for investigating deformations on full-resolution differential SAR interferograms, Geoscience and Remote 1235 Sensing, IEEE Transactions on, 42(7), 1377-1386, doi:10.1109/TGRS.2004.828196. 


\section{A post print of a published manuscript at Computers and Geosciences}

1236 Lauknes, T. R., H. A. Zebker, and Y. Larsen, 2011. InSAR Deformation Time Series Using an $L_{1}$-Norm Small-

1237 Baseline Approach, IEEE Transactions on Geoscience and Remote Sensing, 49(1), 536-546, 1238 doi:10.1109/TGRS.2010.2051951.

1239 Li, Z., E. Fielding, P. Cross, and R. Preusker, 2009. Advanced InSAR atmospheric correction: MERIS/MODIS 1240 combination and stacked water vapour models, International Journal of Remote Sensing, 30(13), 3343-3363, 1241 doi:10.1080/01431160802562172.

1242 Liang, C., Z. Liu, E. J. Fielding, and R. Bürgmann, 2018. InSAR Time Series Analysis of L-Band Wide-Swath SAR 1243 Data Acquired by ALOS-2, IEEE Transactions on Geoscience and Remote Sensing, 56(8), 4492-4506, 1244 doi:10.1109/TGRS.2018.2821150.

1245 Lin, Y. n. N., M. Simons, E. A. Hetland, P. Muse, and C. DiCaprio, 2010. A multiscale approach to estimating 1246 topographically correlated propagation delays in radar interferograms, Geochemistry, Geophysics, Geosystems, 1247 11(9), doi:10.1029/2010GC003228.

1248 Lohman, R. B., and M. Simons, 2005. Some thoughts on the use of InSAR data to constrain models of surface 1249 deformation: Noise structure and data downsampling, Geochemistry, Geophysics, Geosystems, 6(1), 1250 doi:10.1029/2004GC000841.

1251 López-Quiroz, P., M.-P. Doin, F. Tupin, P. Briole, and J.-M. Nicolas, 2009. Time series analysis of Mexico City 1252 subsidence constrained by radar interferometry, Journal of Applied Geophysics, 69(1), 1-15, 1253 doi:10.1016/j.jappgeo.2009.02.006.

1254 Morrison, K., J. C. Bennett, M. Nolan, and R. Menon, 2011. Laboratory Measurement of the DInSAR Response to 1255 Spatiotemporal Variations in Soil Moisture, IEEE Transactions on Geoscience and Remote Sensing, 49(10), 1256 3815-3823, doi:10.1109/TGRS.2011.2132137.

1257 Onn, F., and H. A. Zebker, 2006. Correction for interferometric synthetic aperture radar atmospheric phase artifacts 1258 using time series of zenith wet delay observations from a GPS network, Journal of Geophysical Research: Solid 1259 Earth, $111(\mathrm{~B} 9), \mathrm{n} / \mathrm{a}-\mathrm{n} / \mathrm{a}$, doi:10.1029/2005JB004012.

1260 Parizzi, A., X. Cong, and M. Eineder, 2009. First Results from Multifrequency Interferometry. A comparison of 1261 different decorrelation time constants at L, C, and X Band, ESA Scientific Publications (SP-677), 1-5. 


\section{A post print of a published manuscript at Computers and Geosciences}

1262 Pepe, A., and R. Lanari, 2006. On the extension of the minimum cost flow algorithm for phase unwrapping of 1263 multitemporal differential SAR interferograms, Geoscience and Remote Sensing, IEEE Transactions on, 44(9), 1264 2374-2383, doi:10.1109/TGRS.2006.873207.

1265 Pepe, A., A. B. Ortiz, P. R. Lundgren, P. A. Rosen, and R. Lanari (2011), The Stripmap-ScanSAR SBAS Approach 1266 to Fill Gaps in Stripmap Deformation Time Series With ScanSAR Data, IEEE Transactions on Geoscience and 1267 Remote Sensing, 49(12), 4788-4804, doi:10.1109/TGRS.2011.2167979.

1268 Pepe, A., Y. Yang, M. Manzo, and R. Lanari, 2015. Improved EMCF-SBAS Processing Chain Based on Advanced 1269 Techniques for the Noise-Filtering and Selection of Small Baseline Multi-Look DInSAR Interferograms, $1270 \quad$ Geoscience and Remote Sensing, IEEE Transactions on, PP(99), 1-24, doi:10.1109/TGRS.2015.2396875.

1271 Perissin, D., and T. Wang, 2012. Repeat-pass SAR interferometry with partially coherent targets, Geoscience and 1272 Remote Sensing, IEEE Transactions on, 50(1), 271-280, doi:10.1109/tgrs.2011.2160644.

1273 Rocca, F., 2007. Modeling interferogram stacks, IEEE Transactions on Geoscience and Remote Sensing, 45(10), 1274 3289-3299, doi:10.1109/TGRS.2007.902286.

1275 Rosen, P. A., S. Hensley, G. Peltzer, and M. Simons, 2004. Updated repeat orbit interferometry package released, 1276 Eos Trans. $A G U, 85(5), 47-47$, doi:10.1029/2004EO050004.

1277 Rosen, P. A., E. Gurrola, G. F. Sacco, and H. Zebker, 2012. The InSAR scientific computing environment, paper 1278 presented at EUSAR 2012, 23-26 April 2012.

1279 Rodriguez, E., and J. Martin, 1992. Theory and design of interferometric synthetic aperture radars, paper presented 1280 at IEE Proceedings F (Radar and Signal Processing), IET, doi:10.1049/ip-f-2.1992.0018.

1281 Rousseeuw, P. J., and M. Hubert, 2011. Robust statistics for outlier detection, Wiley Interdisciplinary Reviews: Data 1282 Mining and Knowledge Discovery, 1(1), 73-79, doi:10.1002/widm.2.

1283 Samiei-Esfahany, S., J. E. Martins, F. v. Leijen, and R. F. Hanssen, 2016. Phase Estimation for Distributed 1284 Scatterers in InSAR Stacks Using Integer Least Squares Estimation, IEEE Transactions on Geoscience and 1285 Remote Sensing, 54(10), 5671-5687, doi:10.1109/TGRS.2016.2566604.

1286 Schmidt, D. A., and R. Bürgmann, 2003. Time-dependent land uplift and subsidence in the Santa Clara valley, 1287 California, from a large interferometric synthetic aperture radar data set, Journal of Geophysical Research: Solid $1288 \quad$ Earth, 108(B9), doi:10.1029/2002JB002267. 


\section{A post print of a published manuscript at Computers and Geosciences}

1289 Seymour, M. S., and I. G. Cumming, 1994. Maximum likelihood estimation for SAR interferometry, paper 1290 presented at Geoscience and Remote Sensing Symposium, 1994. IGARSS '94, 8-12 Aug 1994, 1291 doi:10.1109/IGARSS.1994.399711.

1292 Sudhaus, H., and S. Jónsson (2009), Improved source modelling through combined use of InSAR and GPS under 1293 consideration of correlated data errors: application to the June 2000 Kleifarvatn earthquake, Iceland, Geophysical 1294 Journal International, 176(2), 389-404, doi:10.1111/j.1365-246X.2008.03989.x.

1295 Tong, X., D. T. Sandwell, and B. Smith-Konter, 2013. High-resolution interseismic velocity data along the San 1296 Andreas Fault from GPS and InSAR, Journal of Geophysical Research: Solid Earth, 118(1), 369-389, 1297 doi:10.1029/2012JB009442.

1298 Tough, R. J. A., D. Blacknell, and S. Quegan, 1995. A Statistical Description of Polarimetric and Interferometric 1299 Synthetic Aperture Radar Data, Proceedings: Mathematical and Physical Sciences, 449(1937), 567-589, $1300 \quad$ doi:10.1098/rspa.1995.0059.

1301 Werner, C., U. Wegmüller, T. Strozzi, and A. Wiesmann, 2000. Gamma SAR and interferometric processing 1302 software, paper presented at Proceedings of the ERS-Envisat symposium, Gothenburg, Sweden.

1303 Xu, X., and D. T. Sandwell (2019), Towards Absolute Phase Recovery with InSAR: Correcting for Earth Tides and 1304 Phase Unwrapping Ambiguities, IEEE Transactions on Geoscience and Remote Sensing, 1305 doi:10.1109/TGRS.2019.2940207.

1306 Yu, C., Z. Li, and N. T. Penna, 2018. Interferometric synthetic aperture radar atmospheric correction using a GPS1307 based iterative tropospheric decomposition model, Remote Sensing of Environment, 204, 109-121, 1308 doi:10.1016/j.rse.2017.10.038.

1309 Zebker, H. A., and J. Villasenor, 1992. Decorrelation in interferometric radar echoes, Geoscience and Remote $1310 \quad$ Sensing, IEEE Transactions on, 30(5), 950-959, doi:10.1109/36.175330.

1311 


\section{Small baseline InSAR time series analysis: Unwrapping error}

\section{Content of this file}

11 Section S1. Supplemental figure S1 to S9 and table S1.

12 Section S2. Design matrices.

13 Section S3. Decorrelation noise simulation.

14 Section S4. Additional software features

15 Supplemental references.

\section{S1. Supplemental figures and tables}

17 This section provides figures S1 to S9 and table S1. Fig. S1 shows the standard deviation of the

18 interferometric phase as a function of the spatial coherence and number of looks. Fig. S2

19 demonstrates the performance of four weighting functions in different temporal decorrelation

20 settings using the mean RMSE of 10,000 realizations of the inverted phase time-series as a

21 function of the number of looks. Fig. S3 demonstrates the simulation of the unwrapped 
22 interferogram for unwrapping error correction with the bridging method, considering the ground

23 deformation, tropospheric turbulence, phase ramps and decorrelation noise. Fig. S4 shows the

24 output percentage of interferograms with unwrapping errors as a function of the LASSO

25 parameter to find its suitable value range. Fig. S5 demonstrates the necessity of adding the step

26 function during the topographic residual correction in the presence of displacement jump using

27 both simulated and read data. Fig. S6 shows the coherence matrix of Sentinel-1 dataset for GPS

28 stations within Sierra Negra. Fig. S7 shows the estimated residual phase time-series. Fig. S8

29 shows the coherence-based network modification for the Sentinel-1 data used in the discussion

30 of the network redundancy in section 6.3. Fig. S9 compares the displacement time-series from

31 the approaches in GIAnT and MintPy with and without unwrapping error correction and

32 weighted network inversion. Table S1 summaries the information of SAR data used in the paper

33 and their configurations for InSAR stack processing. 

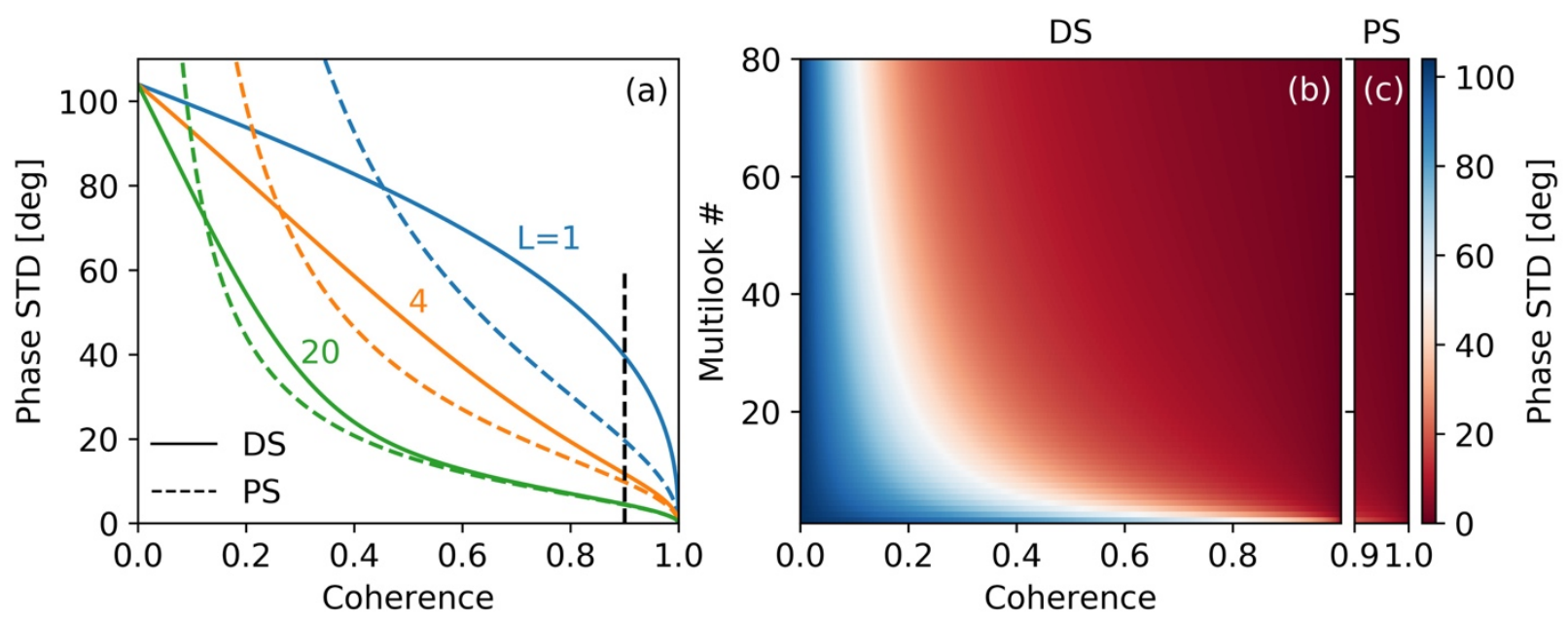

36 Figure S1. Phase standard deviation versus spatial coherence for PS and DS. Related to equation

37 (6). (a) Standard deviation of interferometric phase as function of coherence for DS (solid lines)

38 and PS (dashed lines) with 1, 4 and 20 looks. The black dashed line marks the effective boundary

39 for PS $(0.9<|\gamma| \leq 1)$. (b) Lookup table to convert spatial coherence to phase standard deviation

40 for number of looks in $[1,80]$. 

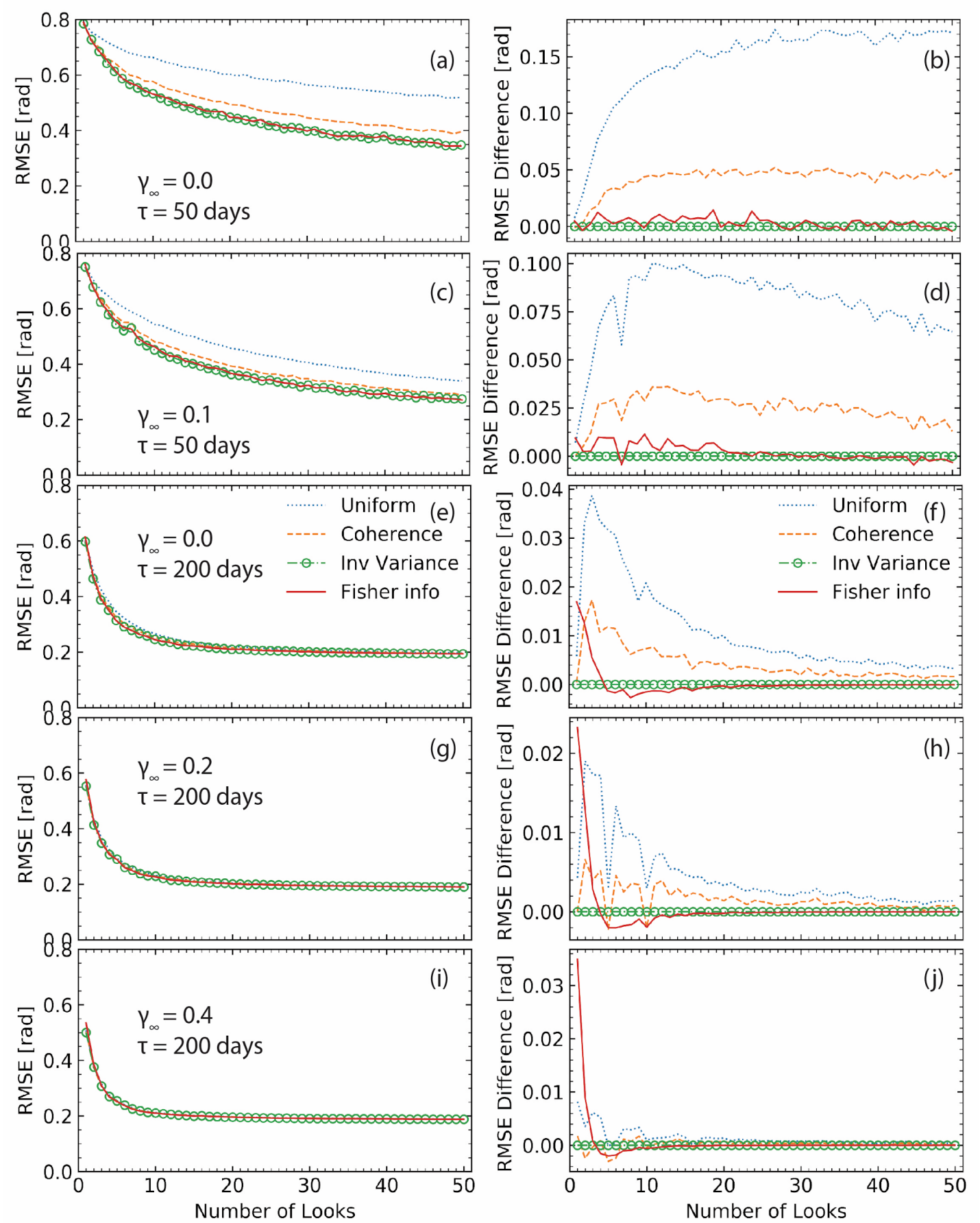

43 Figure S2. Performance indicator for four weighting functions based on (left panel) the mean

44 RMSE of 10,000 realizations of inverted phase time-series as a function of the number of looks.

45 Related to Fig. 1, which uses $\gamma_{\infty}=0.0$ and $\tau=200$ days. Right panel: same as left panel but

46 shown in differential RMSE with respect to inverse-variance weighting. From top to bottom for

47 different temporal decorrelation settings. 


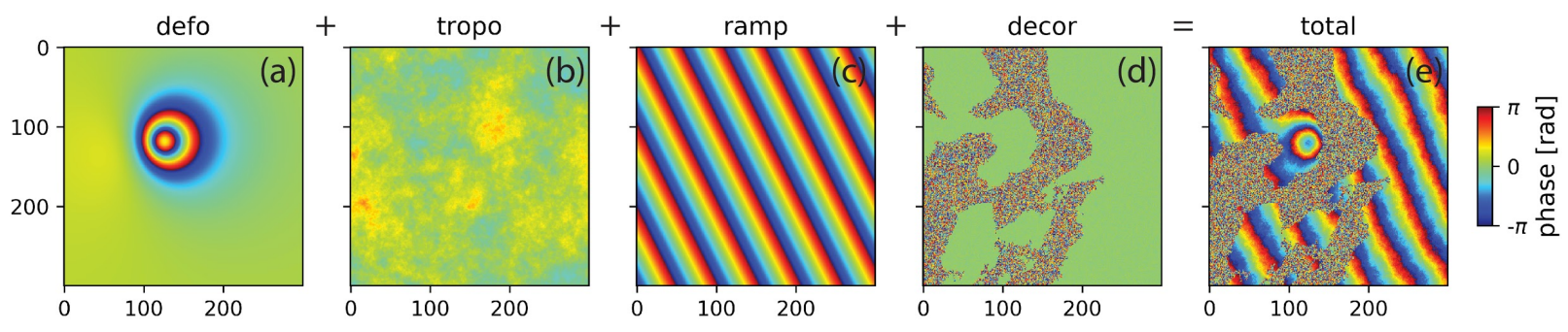

49 Figure S3. Simulate interferogram for unwrapping error correction with the bridging method.

50 Related to Fig. 2. We consider an area of 300 by 300 pixels with spatial resolution of $62 \mathrm{~m}$ in

51 both directions, illustrated by radar echoes in a Sentinel-1-like geometry in descending orbit

52 (with an incidence angle of $34 \mathrm{deg}$ and heading angle of -168 deg). (a) Deformation phase

53 caused by a Mogi source $(x=120 \mathrm{row}, \mathrm{y}=120 \mathrm{col}, \mathrm{z}=2 \mathrm{~km}$ under the free surface with a

54 volume change of $10^{6} \mathrm{~m}^{3}$ ), (b) tropospheric turbulence modeled as an isotropic two-dimensional

55 surface with a power law behavior (the multiplier of spectrum amplitude $\mathrm{p} 0=1 \mathrm{e}-3$, assuming a

56 flat area without stratified tropospheric delay; Hanssen, 2001), (c) phase ramp modeled as a

57 linear surface, and (d) simulated decorrelation noise (see section S3). The water body mask is

58 rescaled from the real DEM in western Kyushu, Japan. We specify the spatial coherence of 0.6

59 and 0.001 for pixels on land and water respectively with the number of looks of 15 by 5 .

60 


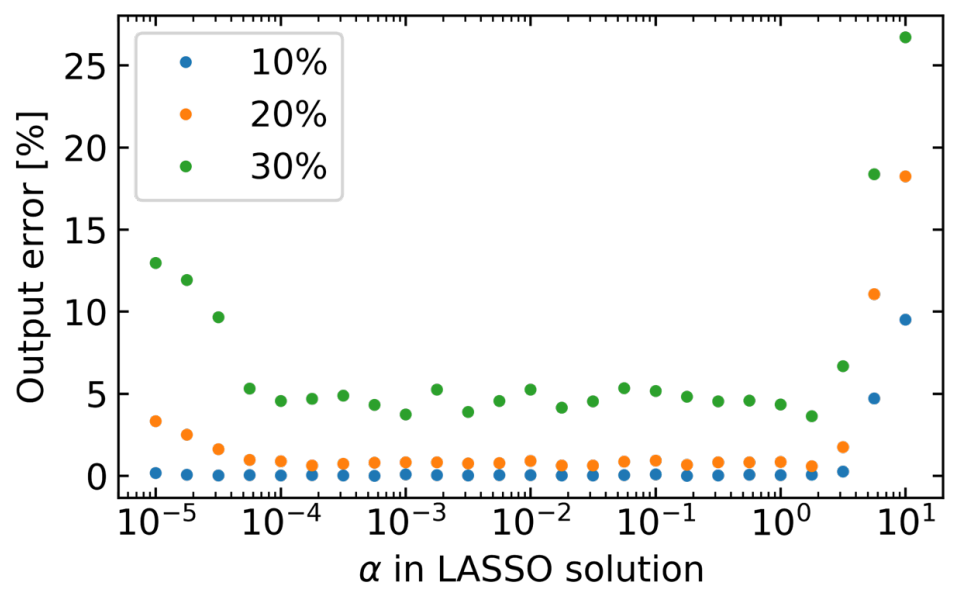

62 Figure S4. Simulation for the optimal LASSO trade-off parameter $\alpha$. Related to equation (11) in

63 section 3.2. Mean output percentage of 100 realization of interferograms with unwrapping errors

64 after correction as a function of the nonnegative $\alpha$ value for different input percentage of

65 interferograms with unwrapping errors. The network of interferograms is the same as Fig. 4a.

66 The simulation result shows that any number of $\alpha$ in $\left[10^{-4}, 10^{0}\right]$ works. We choose $10^{-2}$ as default

67 value.

68 

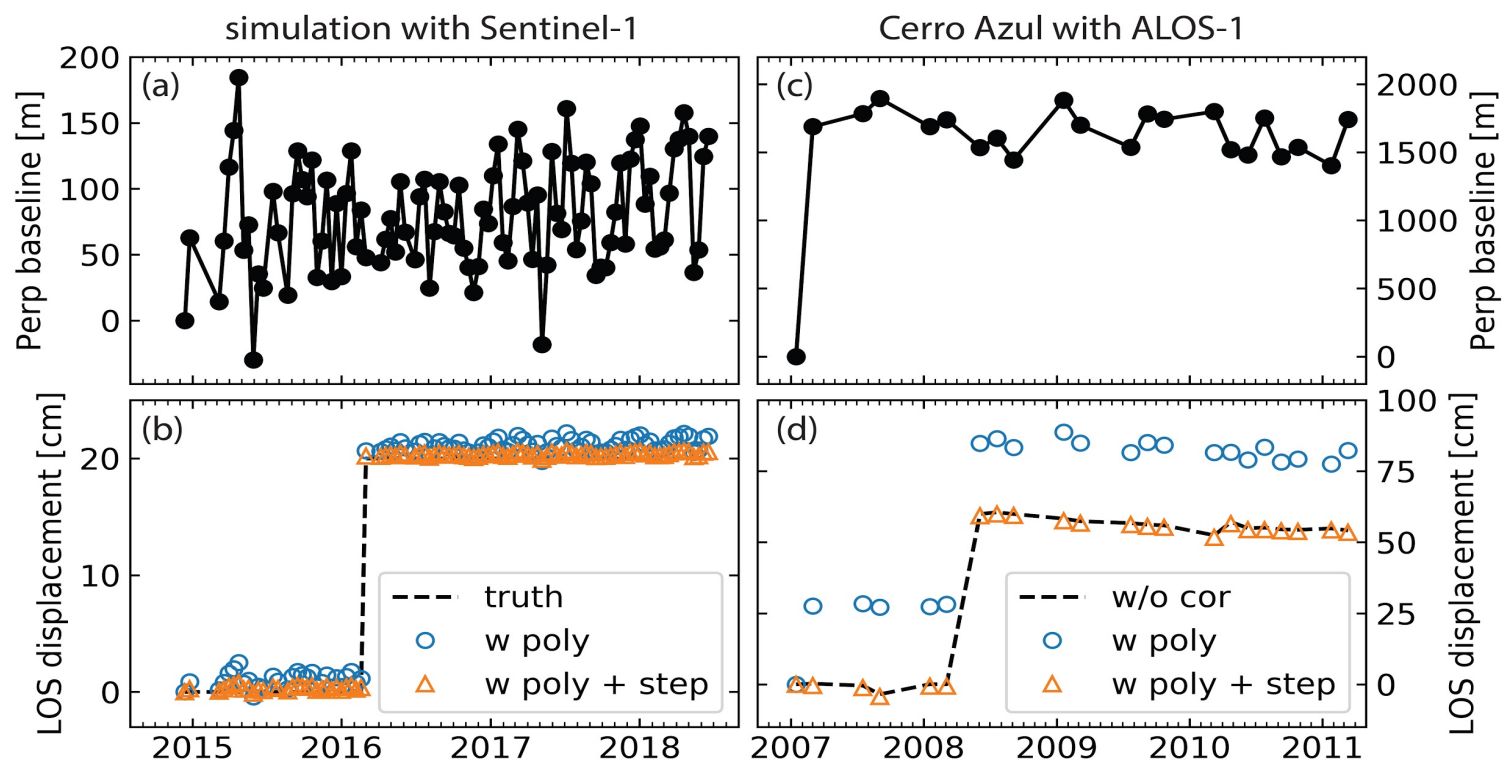

70 Figure S5. Illustration of the step function in topographic residual correction in presence of

71 displacement jumps. Related to equation (13) in section 4.8. (a and b) Perpendicular baseline

72 history (from the Sentinel-1 data of section 5) and an arbitrary displacement time-series using

73 simulated data (with a permanent displacement jump at 1 March 2016 with a magnitude of 20

$74 \mathrm{~cm}$, shown as the dashed black line in (b), in addition to the topographic residual contribution

75 from a DEM error of $50 \mathrm{~m}$ ). Blue empty circles and orange triangles represent displacement

76 time-series after topographic residual correction assuming quadratic model without and with a

77 step function, respectively. (c and d) Same as ( $a$ and b) but (i) using ALOS-1 data for one pixel

78 on Cerro Azul located at [W91.270, $\left.\mathrm{S} 0.928^{\circ}\right]$ and (ii) the black dashed line for the displacement

79 time-series without topographic residual correction. In both simulated and real data, the

80 disagreement between the low-frequency quadratic model and the high-frequency displacement

81 jump leads to biased estimation of the topographic residual (Du et al., 2007) and adding a step

82 function could effectively eliminate this estimation bias. This estimation bias is amplified in the

83 first ALOS-1 acquisition by its large perpendicular baseline (the difference between black

84 dashed line and the blue empty circles in (d)). 

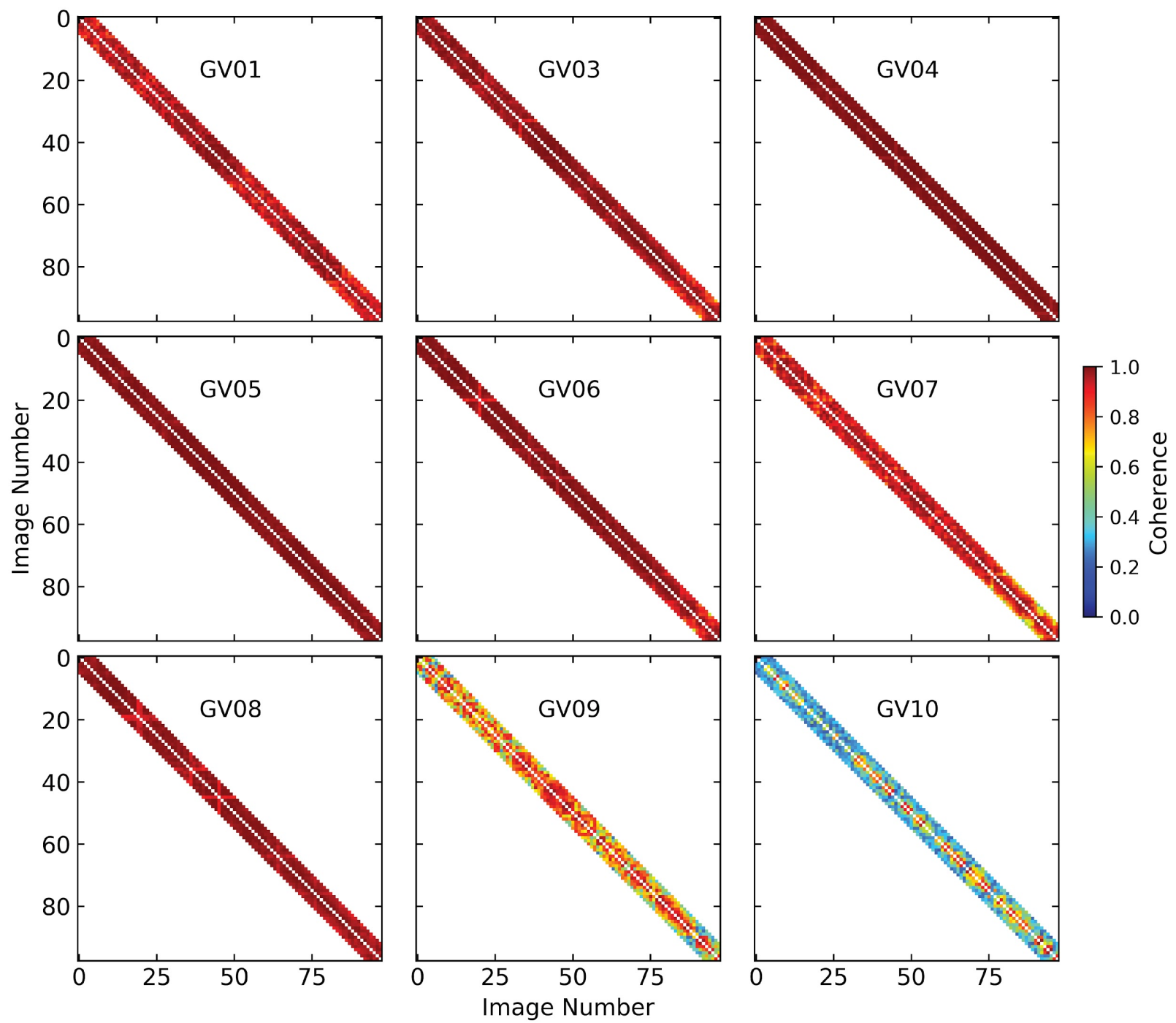

86 Figure S6. Coherence matrix of Sentinel-1 dataset for GPS stations within Sierra Negra caldera.

87 Related to Fig. 8 in section 5.1. Both $\mathrm{X}$ and $\mathrm{Y}$ axis indicate the number of SAR acquisitions.

88 Station GV10 is located in a densely vegetated area outside the caldera on the rim, resulting in

89 fast decorrelation with low spatial coherence on interferograms with more than 2 lags. 


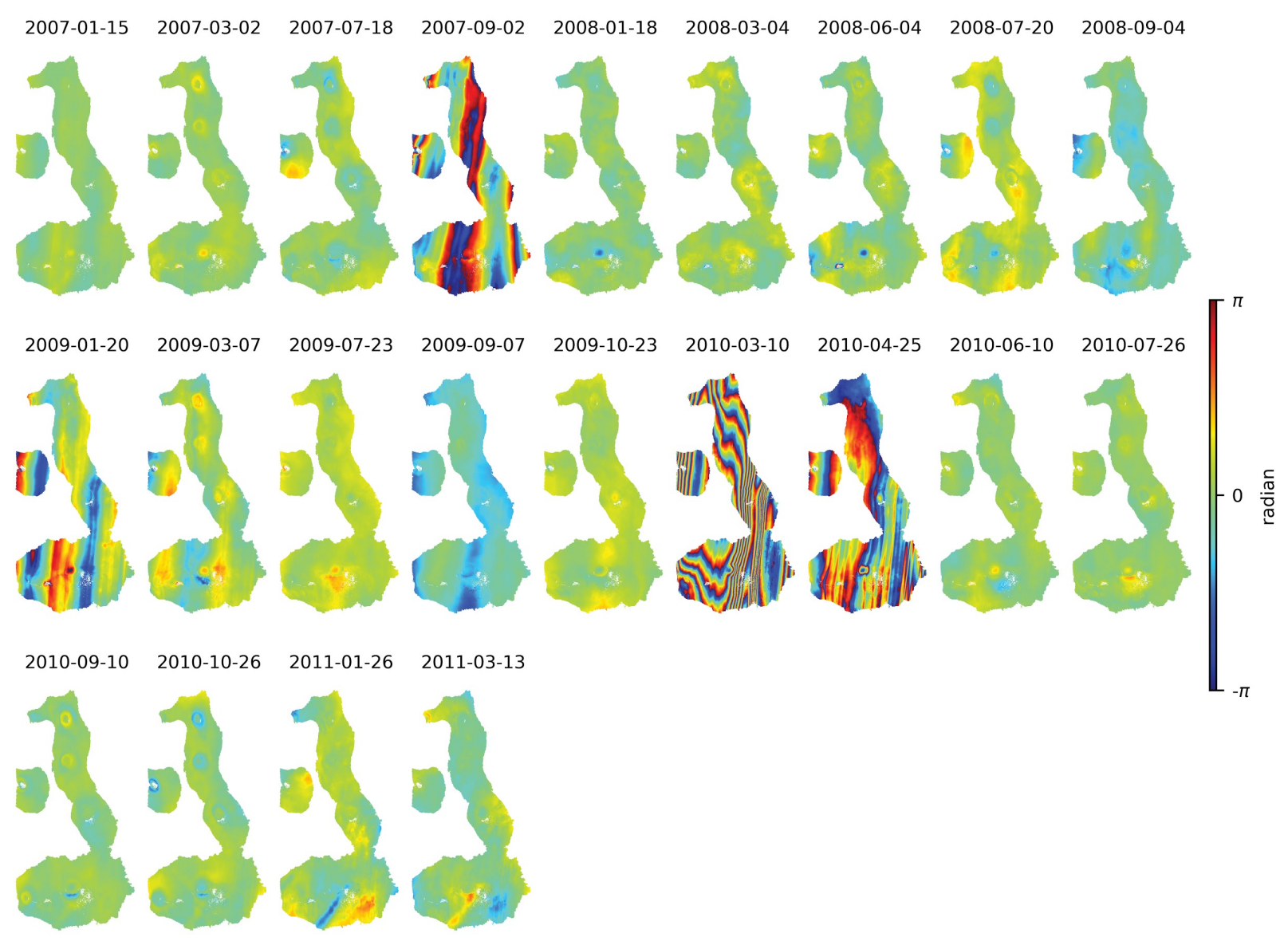

92 Figure S7. The estimated residual phase time-series $\hat{\phi}_{\text {resid }}$ of ALOS-1 dataset. Related to 93 equation (13-14) in section 4.7 and Fig. 12 in section 5.4. A quadratic phase ramp has been 94 estimated and removed from each acquisition. This is used in equation (14) to calculate the 95 residual phase RMS value. Phases on 2 September 2007, 10 March 2010 and 25 April 2010 are 96 severely contaminated by ionospheric streaks and are automatically identified as outliers. Phase 97 on 20 January 2009 is contaminated by ionosphere also but is not identified as outlier due to its 98 relatively small magnitude. 

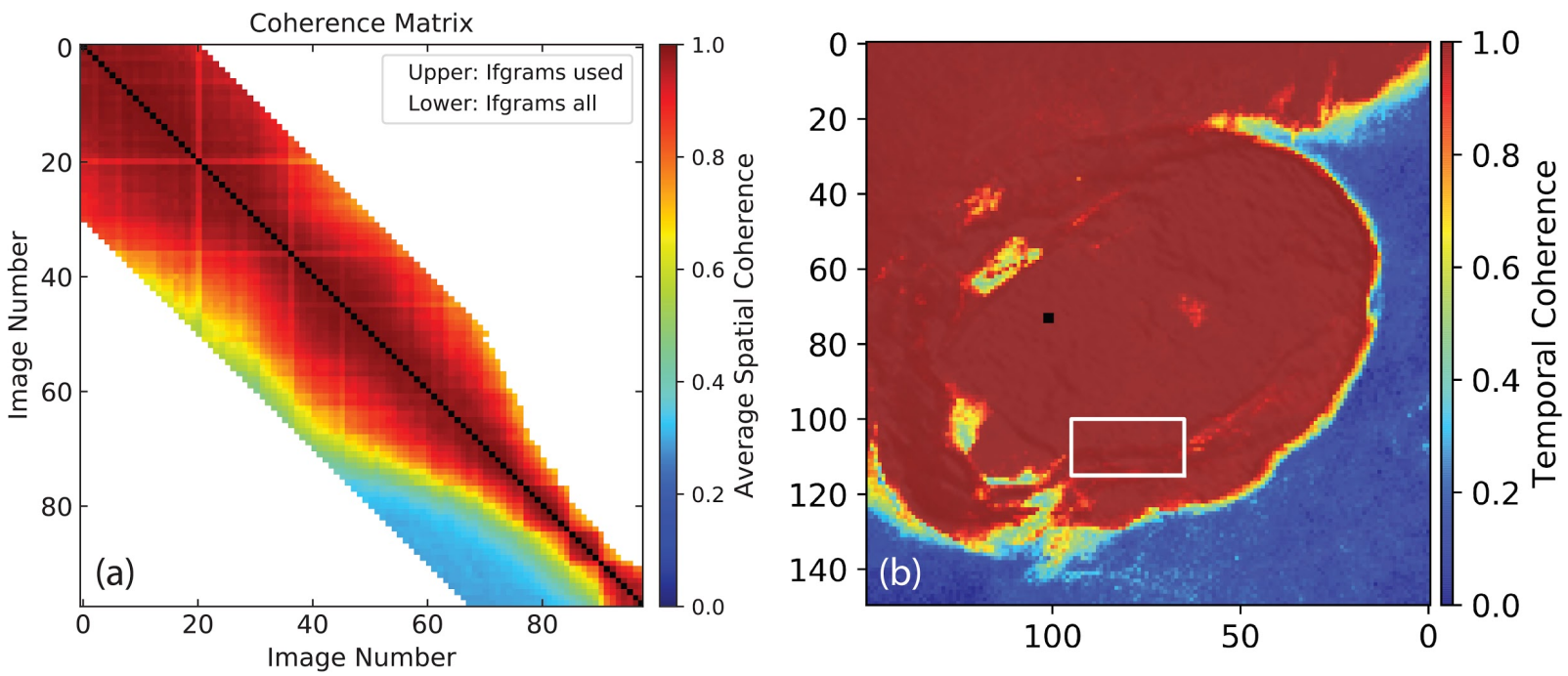

101 Figure S8. Coherence-based network modification for Sentinel-1 data used in section 6.3 in

102 Sierra Negra. Related to Fig. 14 in section 6.3. (a) Coherence matrix of the customized area of

103 interest along the trap door fault within Sierra Negra caldera (marked by the white rectangle in

104 (b)). A network of interferograms with 30 sequential connections (2475 in total) are generated 105 from 98 SAR acquisitions, as shown in the lower triangle. The upper triangle shows the

106 interferogram kept after the network modification. A maximum of 20 connections are shown in 107 Fig. 14 only. (b) Temporal coherence of the network inversion from the interferogram stack with 108 a maximum of 20 connections. 

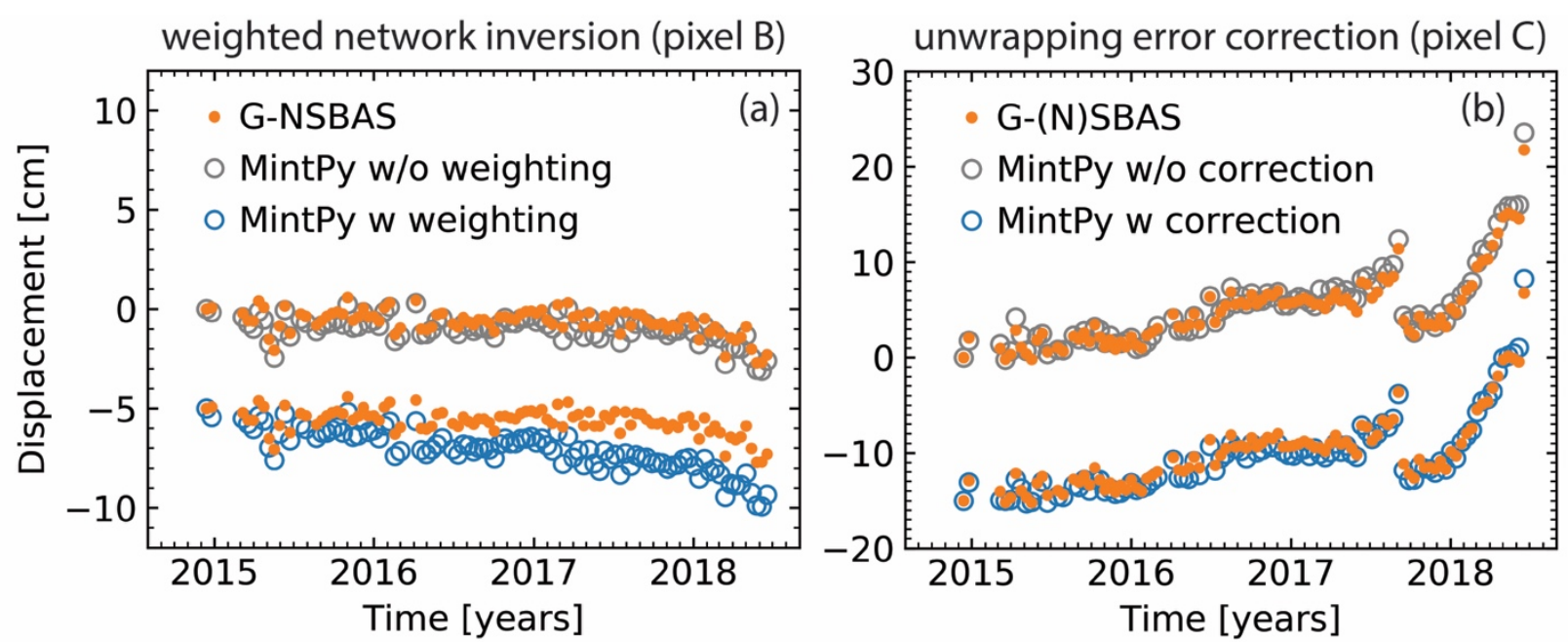

111 Figure S9. Impact of (a) weighted network inversion and (b) unwrapping error correction on the

112 displacement time-series. Related to Fig. 16 in section 6.5. The comparison within (a) shows that

113 the difference on pixel B (Alcedo's flank) between MintPy and G-NSBAS is caused by the

114 weighting during the network inversion. The comparison within (b) shows that the difference on

115 pixel C (Fernandina's crater) between MintPy and G-(N)SBAS is caused by the unwrapping 116 error correction. 
118 Table S1. SAR dataset information with parameters used in InSAR stack processing

\begin{tabular}{|c|c|c|}
\hline Satellite & ALOS-1 & Sentinel-1A/B \\
\hline Orbit direction & Ascending & Descending \\
\hline Track number & 133 & 128 (swath $1 \& 2$ ) \\
\hline $\begin{array}{l}\text { Start / end date } \\
\text { (\# of acquisitions) }\end{array}$ & $\begin{array}{c}2007-01-15 / 2011-03-13 \\
(22)\end{array}$ & $\begin{array}{c}2014-12-13 / 2018-06-19 \\
(98)\end{array}$ \\
\hline $\begin{array}{l}\text { Network selection criteria } \\
\text { (\# of Interferograms) }\end{array}$ & $\begin{array}{c}\mathrm{B}_{\text {temp }} \leq 1800 \text { days } \\
\mathrm{B}_{\perp} \leq 1800 \mathrm{~m} \\
(228)\end{array}$ & $\begin{array}{c}\text { Sequential with } 5 \text { connections } \\
\qquad(475)\end{array}$ \\
\hline $\begin{array}{c}\text { \# of looks in range / azimuth } \\
\text { direction }\end{array}$ & $8 \times 16$ & $15 \times 5$ \\
\hline $\begin{array}{l}\text { Ground pixel size in range / } \\
\text { azimuth direction (m) }\end{array}$ & $60 \times 51$ & $62 \times 70$ \\
\hline InSAR Processor & ROI_PAC & ISCE \\
\hline Phase Unwrapping & SNAPHU & SNAPHU \\
\hline
\end{tabular}




\section{S2. Design matrices}

121 This section shows examples to generate the design matrices used in the software. A demo set of

$122 N=8 \mathrm{SAR}$ images acquired at $\left[t_{1}, \ldots, t_{8}\right]$ is used as the example. A stack of $M=18$ interferograms

123 is selected using the sequential method with 3 connections. An earthquake or volcanic eruption

124 event occurred between $t_{6}$ and $t_{7}$ (red dashed line), which caused a permanent ground 125 displacement offset.

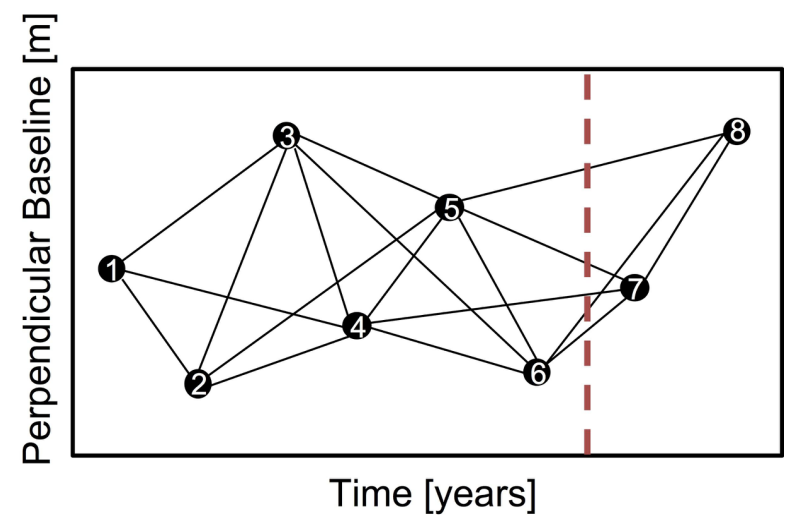

127 Figure S10. Network configuration of the demo dataset. Red dashed line marks the time of a 128 displacement offset due to an earthquake or volcanic eruption.

\section{S2.1 Network inversion}

130 To generate the design matrix $\boldsymbol{A}$ for network inversion used in equation (1) in section 2.1, we

131 first generate an $M \times N$ matrix. For each row, it consists $-1,0$ and 1 with -1 for the reference

132 acquisition, 1 for the secondary acquisition and 0 for the rest. Due to the relative nature of

133 InSAR measurement, the phase on the reference date (the first date by default) cannot be

134 resolved, thus, we can only solve $\left[\phi^{2}, \ldots, \phi^{N}\right]$ instead of $\left[\phi^{1}, \ldots, \phi^{N}\right]$ and the corresponding

135 column (the first column by default) is eliminated in the design matrix $\boldsymbol{A}$, which results in size of $136 M \times(N-1)$. 


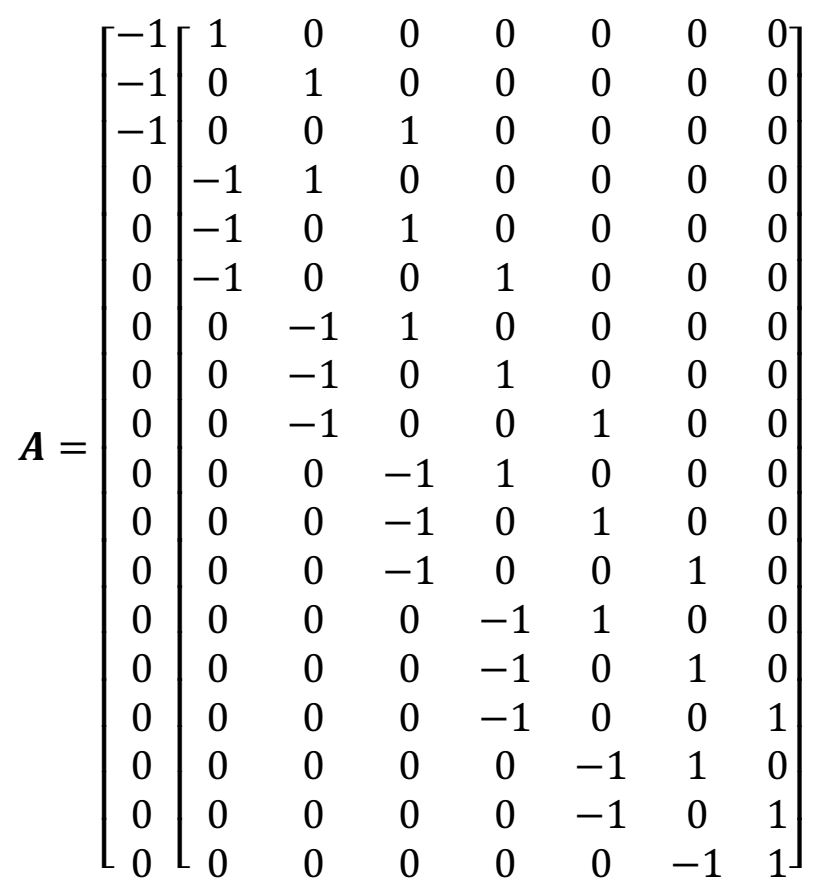

141 Design matrix $\boldsymbol{C}$ describe the combination of interferograms to form the triplets used in equation

142 (10) in section 3.2 for the phase closure unwrapping error correction. An example of $\boldsymbol{C}$ is shown

143 below based on the demo network with number of triplets $T=16$. 


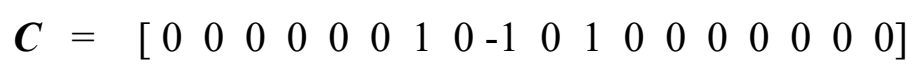

161 Design matrix $\boldsymbol{G}$ is used in equation (13) for topographic residual correction in section 4.8. It is 162 in size of $N \times\left(1+N_{\text {poly }}+N_{\text {step }}\right)$, where $N_{\text {poly }}$ is the user-defined polynomial order $N_{\text {poly }}$ (2 by 163 default), $N_{\text {step }}$ is the number of Heaviside step functions ( 0 by default) describing offsets at 164 specific prior selected times. An example of $\boldsymbol{G}$ is shown below based on the demo network. 165

$$
\boldsymbol{G}=\left[\begin{array}{lllll}
\frac{4 \pi}{\lambda} \frac{B_{\perp}^{1}}{r \sin (\theta)} & 1 & \left(t_{1}-t_{1}\right) & \frac{\left(t_{1}-t_{1}\right)^{2}}{2} & 0 \\
\frac{4 \pi}{\lambda} \frac{B_{\perp}^{2}}{r \sin (\theta)} & 1 & \left(t_{2}-t_{1}\right) & \frac{\left(t_{2}-t_{1}\right)^{2}}{2} & 0 \\
\frac{4 \pi}{\lambda} \frac{B_{\perp}^{3}}{r \sin (\theta)} & 1 & \left(t_{3}-t_{1}\right) & \frac{\left(t_{3}-t_{1}\right)^{2}}{2} & 0 \\
\frac{4 \pi}{\lambda} \frac{B_{\perp}^{4}}{r \sin (\theta)} & 1 & \left(t_{4}-t_{1}\right) & \frac{\left(t_{4}-t_{1}\right)^{2}}{2} & 0 \\
\frac{4 \pi}{\lambda} \frac{B_{\perp}^{5}}{r \sin (\theta)} & 1 & \left(t_{5}-t_{1}\right) & \frac{\left(t_{5}-t_{1}\right)^{2}}{2} & 0 \\
\frac{4 \pi}{\lambda} \frac{B_{\perp}^{6}}{r \sin (\theta)} & 1 & \left(t_{6}-t_{1}\right) & \frac{\left(t_{6}-t_{1}\right)^{2}}{2} & 0 \\
\frac{4 \pi}{\lambda} \frac{B_{\perp}^{7}}{r \sin (\theta)} & 1 & \left(t_{7}-t_{1}\right) & \frac{\left(t_{7}-t_{1}\right)^{2}}{2} & 1 \\
\frac{4 \pi}{\lambda} \frac{B_{\perp}^{8}}{r \sin (\theta)} & 1 & \left(t_{8}-t_{1}\right) & \frac{\left(t_{8}-t_{1}\right)^{2}}{2} & 1
\end{array}\right]
$$


167 Then equation (13) can be formed as a linear system with $N$ equations as below:

168

169

$$
\hat{\phi}-\hat{\phi}_{\text {tropo }}=\boldsymbol{G} X+\phi_{\text {resid }}
$$

171 where $X=\left[z_{\varepsilon}, c_{0}, c_{1}, c_{2}, s_{7}\right]^{T}$ is the vector of unknown parameters, $\hat{\phi}, \hat{\phi}_{\text {tropo }}$ and $\phi_{\text {resid }}$ are the

$172 N \times 1$ inverted raw phase time-series, estimated tropospheric delay time-series and residual 173 phase time-series, respectively. We apply the least squares estimation to obtain the solution as:

$$
\begin{gathered}
\hat{X}=\left(\boldsymbol{G}^{T} \boldsymbol{G}\right)^{-1} \boldsymbol{G}^{T}\left(\hat{\phi}-\hat{\phi}_{\text {tropo }}\right) \\
\hat{\phi}_{\text {resid }}=\hat{\phi}-\hat{\phi}_{\text {tropo }}-\boldsymbol{G} \hat{X}
\end{gathered}
$$

178 The estimated residual phase $\hat{\phi}_{\text {resid }}$ is used to characterize the noise of phase time-series using 179 equation (14) in section 4.9. The noise-reduced displacement time-series is given as:

$$
\phi_{\text {dis }}^{i}=\hat{\phi}^{i}-\hat{\phi}_{\text {tropo }}^{i}-\frac{-4 \pi}{\lambda} \frac{B_{\perp}^{i}}{r \sin (\theta)} \hat{z}_{\varepsilon}
$$

182

183 where $i=1, \ldots, N$ and $\hat{z}_{\varepsilon}$ is the estimated DEM error in $\hat{X}$.

\section{$184 \quad$ S2.4 Average velocity estimation}

185 For each pixel, the average velocity is estimated as $d^{i}=v t_{i}+c$, where $d^{i}=-\frac{\lambda}{4 \pi} \phi_{d i s}^{i}$ is the

186 displacement at $t_{i}$ in meters, $v$ is the unknown velocity and $c$ is the unknown offset. The solution

187 can be obtained using least squares approximation. An example of the design matrix $\boldsymbol{E}$ is shown 188 below based on the demo network. 
190

$$
\boldsymbol{E}=\left[\begin{array}{ll}
t_{1}-t_{1} & 1 \\
t_{2}-t_{1} & 1 \\
t_{3}-t_{1} & 1 \\
t_{4}-t_{1} & 1 \\
t_{5}-t_{1} & 1 \\
t_{6}-t_{1} & 1 \\
t_{7}-t_{1} & 1 \\
t_{8}-t_{1} & 1
\end{array}\right]
$$

192 For linear displacement, the uncertainty of the estimated velocity $\sigma_{v}$ is given by equation (10) in 193 Fattahi and Amelung (2015) as:

194

195

$$
\sigma_{v}=\sqrt{\frac{\sum_{i=1}^{N}\left(\phi_{d i s}^{i}-\widehat{\phi}_{d i s}^{i}\right)^{2}}{(N-2) \sum_{i=1}^{N}\left(t_{i}-\bar{t}\right)^{2}}}
$$

196

197 where $\hat{\phi}_{\text {dis }}^{i}$ is the predicted linear displacement at $i_{\text {th }}$ acquisition $\bar{t}$ is the mean value of time in 198 years.

199 


\section{S3. Decorrelation noise simulation}

\section{S3.1 Coherence model}

202 We simulate the coherence for a stack of interferograms on one pixel using a decorrelation

203 model with exponential decay for temporal decorrelation. The spatial coherence $\gamma^{j}$ of the $j_{\text {th }}$

204 interferogram can be expressed as (Zebker and Villasenor, 1992; Hanssen, 2001; Parizzi et al., 205 2009):

$$
\gamma=\gamma_{\text {geom }} \cdot \gamma_{D C} \cdot \gamma_{\text {temporal }}
$$

209 where $\gamma_{\text {geom }}$ represents the geometric decorrelation, $\gamma_{D C}$ represents the Doppler centroid 210 decorrelation, $\gamma_{\text {temporal }}$ represents the temporal decorrelation, given by the equations below.

211 Note that the thermal decorrelation $\gamma_{\text {thermal }}$ is served as the instantaneous decorrelation in

212 temporal decorrelation $\gamma_{\text {temporal }}$ (Parizzi et al., 2009).

$$
\gamma_{\text {geom }}= \begin{cases}1-\frac{\left|B_{\perp}\right|}{B_{\perp}^{\text {crit }}}, & \left|B_{\perp}\right| \leq B_{\perp}^{\text {crit }} \\ 0, & \left|B_{\perp}\right|>B_{\perp}^{\text {crit }}\end{cases}
$$

$$
\gamma_{D C}= \begin{cases}1-\frac{\left|\Delta f_{D C}\right|}{B_{a z}}, & \left|\Delta f_{D C}\right| \leq B_{a z} \\ 0, & \left|\Delta f_{D C}\right|>B_{a z}\end{cases}
$$

$$
\gamma_{\text {temporal }}(t)=\left(\gamma_{\text {thermal }}-\gamma_{\infty}\right) e^{-t / \tau}+\gamma_{\infty}
$$

$$
\gamma_{\text {thermal }}=\frac{1}{1-S N R^{-1}}
$$


219 The critical perpendicular baseline $B_{\perp}^{\text {crit }}=\lambda \frac{B_{r g}}{c} R \cdot \tan (\theta)$ is the baseline causing a spectral 220 shift equal to the radar bandwidth $B_{r g}$ in range direction (Zebker and Villasenor, 1992; Hanssen, 221 2001), where $\lambda$ is the radar wavelength, $c$ is the speed of light, $R$ is the distance between radar 222 antenna and ground target and $\theta$ is the incidence angle, $S N R$ is the thermal signal-to-noise ratio 223 of radar receiver. $\tau$ is the time constant which depends on radar wavelength $\lambda$, it's the time for 224 coherence to drop down to 1/e, i.e. 0.36, from its initial value (Parizzi et al., 2009; Rocca, 2007). $225 \gamma_{\infty}$ is the long-term coherence, or minimum attainable coherence value, which converged over 226 time, usually with high values in urban area and low values in vegetated area. Note that this 227 model does not consider the seasonal behavior of temporal decorrelation, volume decorrelation, 228 and processing-induced decorrelation. For a given set of SAR acquisitions, the geometric and 229 Doppler centroid decorrelation is almost constant among all pixels. All parameters are deployed 230 with typical parameters of Sentinel-1 SAR sensor.

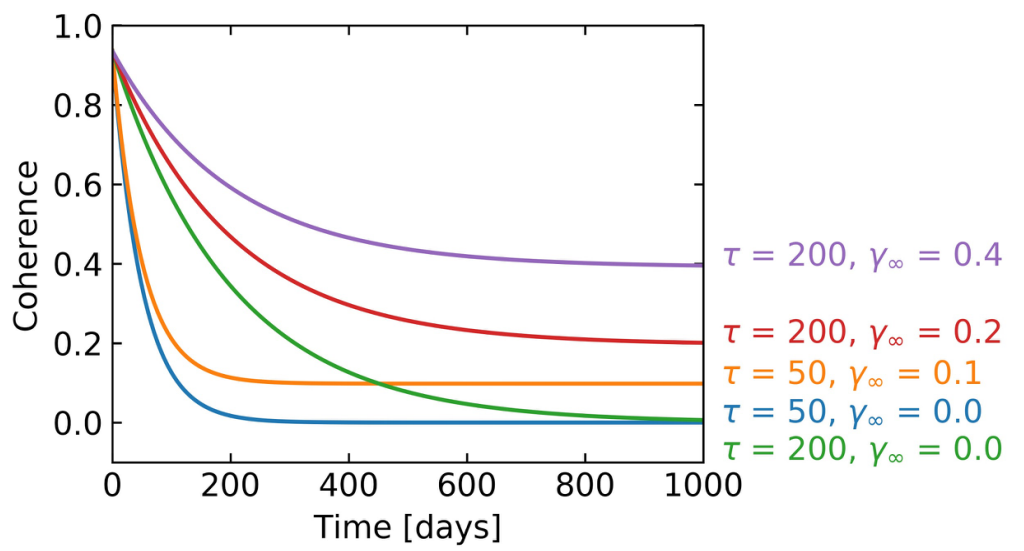

233 Figure S11. Simulated coherence as a function of temporal baseline, color coded by different $\tau$ 234 and $\gamma_{\infty}$ settings used in Fig. S2. 
236 For distributed scatterers (DS) in natural, vegetated terrain the interferometric phase exhibits

237 highly unpredictable speckle characteristics. Its phase can be appropriately modeled by a random 238 process, complex, stationary, circular Gaussian process in the case of SAR image. Applying the 239 central limit theorem, the probability density function $p d f(\Delta \phi)$ of interferometric phase is 240 obtained as (equation (66) from Tough et al., 1995; equation (4.2.23) from Hanssen, 2001):

$$
p d f(\Delta \phi)=\frac{\left(1-|\gamma|^{2}\right)^{L}}{2 \pi}\left\{\frac{\Gamma(2 L-1)}{[\Gamma(L)]^{2} 2^{2(L-1)}} \times\left[\frac{(2 L-1) \beta}{\left(1-\beta^{2}\right)^{L+\frac{1}{2}}}\left(\frac{\pi}{2}+\arcsin \beta\right)+\frac{1}{\left(1-\beta^{2}\right)^{L}}\right]+D\right\}
$$

244 where $\beta=|\gamma| \cos \left(\Delta \phi-\Delta \phi_{0}\right)$, expected interferometric phase $\Delta \phi_{0}=E\{\Delta \phi\}$, gamma function

$245 \Gamma(L)=\int_{0}^{\infty} t^{L-1} e^{-t} d t$, for $L \in R$ and $D$ a finite summation term. Note that $D$ vanishes for 246 single-look datasets $(L=1)$.

248 The 10,000 realizations/samples of decorrelation noise of each interferogram (used in section 249 2.4) is simulated by generating a distribution given by equation (S15) with corresponding 250 coherence $\gamma$ and number of looks $L$. One example with $\gamma=0.1$ and $L=9 \times 3$ is shown in Fig. 251 S12. 


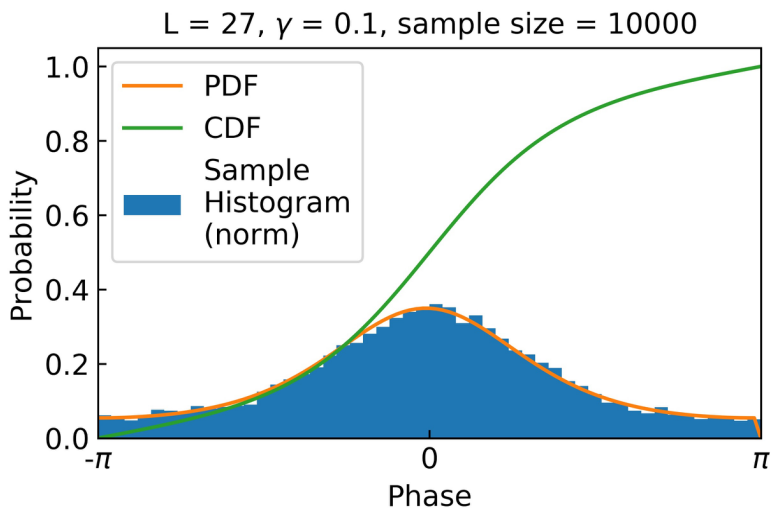

254 Figure S12. Sampling the decorrelation noise based on phase PDF of distributed scatterers.

255 Blue bars: normalized histogram of sampled decorrelation noises. Orange and green solid line:

256 phase PDF and cumulative distribution function.

257
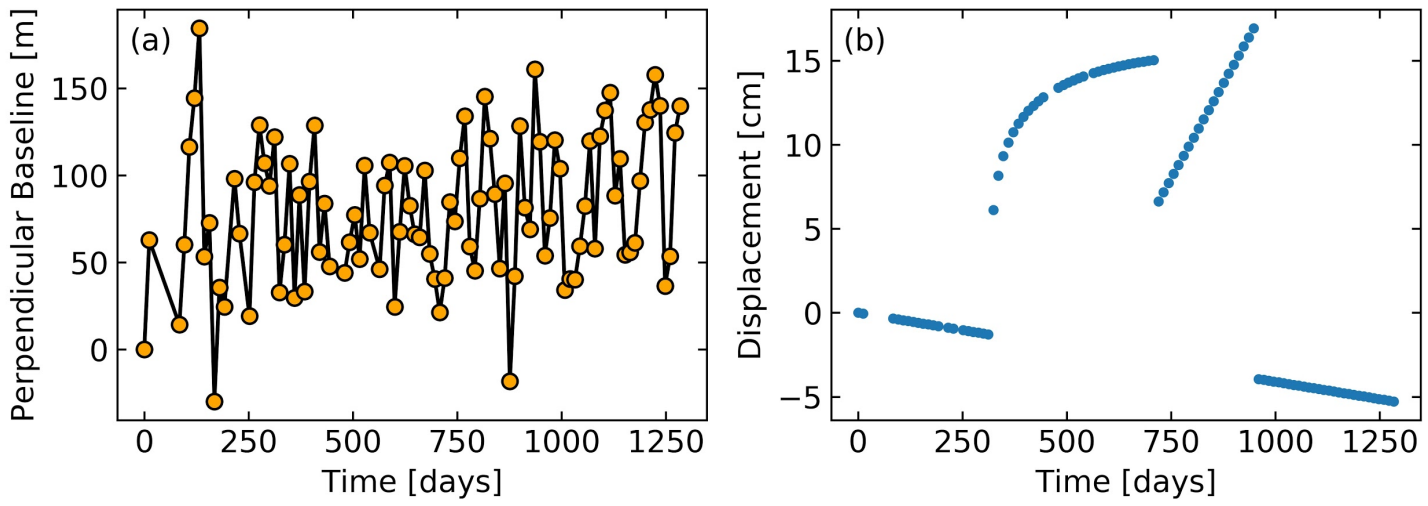

Figure S13. Time-series configuration for simulation. (a) Perpendicular baseline history from

260 the 98 Sentinel-1 images of section 5. (b) Specified time-dependent displacement used in section

$261 \quad 2.4$ and 3.2. 


\section{S4. Additional software features}

264 S4.1 Customized workflow beyond smallbaselineApp.py

265 Most scripts in MintPy are stand-alone (summarized in Table S4). Users can apply any phase 266 correction at any time to evaluate the impact. Fig. S14 shows an example, where we use 267 individual scripts (link on GitHub) to compare velocities estimated from displacement time268 series with different tropospheric delay correction methods on Alcedo volcano.
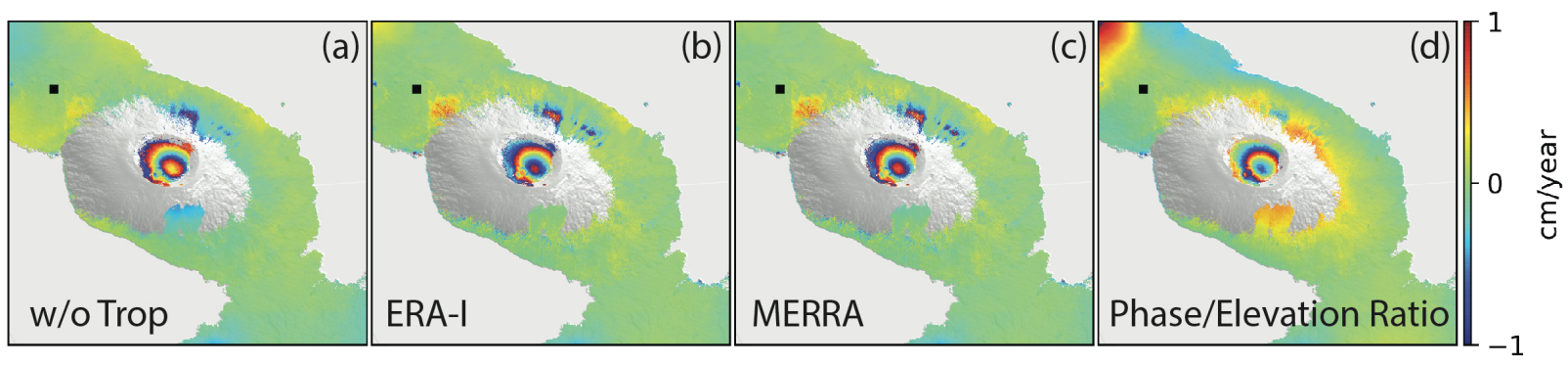

271 Figure S14. Deformation velocity maps on Alcedo volcano from Sentinel-1 (a) without

272 tropospheric correction, with tropospheric correction using (b) ERA-Interim, (c) MERRA-2 and

273 (d) the empirical phase-elevation ratio method.

274

Table S4. Stand-alone scripts in MintPy

\begin{tabular}{|l|l|}
\hline add.py & Generate the sum of multiple input files \\
\hline asc_desc2horz_vert.py & Project ascending and descending displacement in LOS \\
& direction to horizontal and vertical direction \\
\hline dem_error.py & DEM error (topographic residual) correction \\
\hline diff.py & Generate the difference of two input files \\
\hline
\end{tabular}




\begin{tabular}{|c|c|}
\hline generate_mask.py & Generate mask file from input file \\
\hline geocode.py & $\begin{array}{l}\text { Resample radar-coded files into geo coordinates, or vice } \\
\text { versa. }\end{array}$ \\
\hline ifgram_inversion.py & Invert network of interferograms into time-series. \\
\hline image_reconstruction.py & Reconstruct network of interferograms from time-series \\
\hline image_math.py & Basic mathematic operation of input file(s) \\
\hline info.py & Display metadata / structure of input file \\
\hline load_data.py & Load a stack of interferograms into HDF5 files \\
\hline load_gbis.py & Load the inversion result from GBIS software \\
\hline load_hdf5.py & Load the binary file(s) into an HDF5 file \\
\hline local_oscillator_drift.py & Correct local oscillator drift for Envisat data \\
\hline mask.py & $\begin{array}{l}\text { Mask input data file with input mask file by setting } \\
\text { values on the unselected pixels into Nan or zero. }\end{array}$ \\
\hline match.py & $\begin{array}{l}\text { Merge two or more geocoded files which share common } \\
\text { area into one file. }\end{array}$ \\
\hline modify_network.py & Modify the network setting of an ifgramStack HDF5 file. \\
\hline multilook.py & Multilook input file. \\
\hline plot_coherence_matrix.py & Plot the coherence matrix of one pixel, interactively. \\
\hline plot_network.py & Plot the network configuration of an ifgramStack file. \\
\hline plot_transection.py & Plot the value of 2D matrix along a profile. \\
\hline prep_aria.py & Prepare input data from ARIA GNUW products \\
\hline prep_gamma.py & Prepare metadata file for GAMMA files. \\
\hline prep_giant.py & Prepare metadata file for GIAnT files. \\
\hline
\end{tabular}




\begin{tabular}{|c|c|}
\hline prep_isce.py & Prepare metadata file for ISCE files. \\
\hline prep_roipac.py & Prepare metadata file for ROI_PAC files. \\
\hline prep_snap.py & Prepare metadata file for SNAP geocoded products. \\
\hline reference_date.py & Change the reference date of a time-series HDF5 file. \\
\hline reference_point.py & Change the reference pixel of an input file. \\
\hline remove_ramp.h5 & Remove phase ramps for input file. \\
\hline save_gbis.py & Save input files in GBIS *.mat file format. \\
\hline save_gmt.py & Save input file in GMT *.grd file format. \\
\hline save_hdfeos5.py & Save input time-series into HDF-EOS5 format. \\
\hline save_kmz.py & Save input file into Google Earth raster image. \\
\hline save_kmz_timeseries.h5 & Save input file into Google Earth points, interactively. \\
\hline save_roipac.py & Save input file into ROI_PAC style binary file format. \\
\hline select_network.py & Select interferometric pairs from input baseline file. \\
\hline smallbaselineApp.py & $\begin{array}{l}\text { Routine time series analysis for small baseline InSAR } \\
\text { stack. }\end{array}$ \\
\hline spatial_average.py & Calculate average in space domain. \\
\hline spatial_filter.py & Spatial filtering of input file. \\
\hline subset.py & Generate a subset of (crop) input file. \\
\hline temporal_average.py & Calculate average in time domain. \\
\hline temporal_derivative.py & $\begin{array}{l}\text { Calculate the temporal derivative of displacement time- } \\
\text { series. }\end{array}$ \\
\hline temporal_filter.py & $\begin{array}{l}\text { Smooth time-series in time domain with a moving } \\
\text { Gaussian window }\end{array}$ \\
\hline
\end{tabular}




\begin{tabular}{|l|l|}
\hline timeseries2velocity.py & Invert time-series for the average velocity. \\
\hline timeseries_rms.py & Calculate the root mean square for each acquisition of the \\
input time-series file. \\
\hline tropo_phase_elevation.py & Correct stratified tropospheric delay based on the \\
\hline tropo_pyaps.py & empirical phase/elevation ratio method. \\
\hline tsview.py & Correct tropospheric delay estimated from global \\
\hline unwrap_error_bridging $\cdot$ py & atmospheric model (GAM) using PyAPS software \\
\hline unwrap_error_- & (Jolivet et al., 2011; 2014). \\
\hline view.py & Interactive time-series viewer. \\
\hline
\end{tabular}

277 S4.2 Filters tools in space and time domain

278 The software supports filters in space or time domain built on skimage (van der Walt et al., 279 2014). Although filtering is not applied in the routine workflow, it is a useful tool to examine the 280 deformation signal because it allows removing undesired signals. Fig. S15 shows an example, 281 where we use spatial Gaussian filtering to confirm a patchy, rapid subsidence signal. 


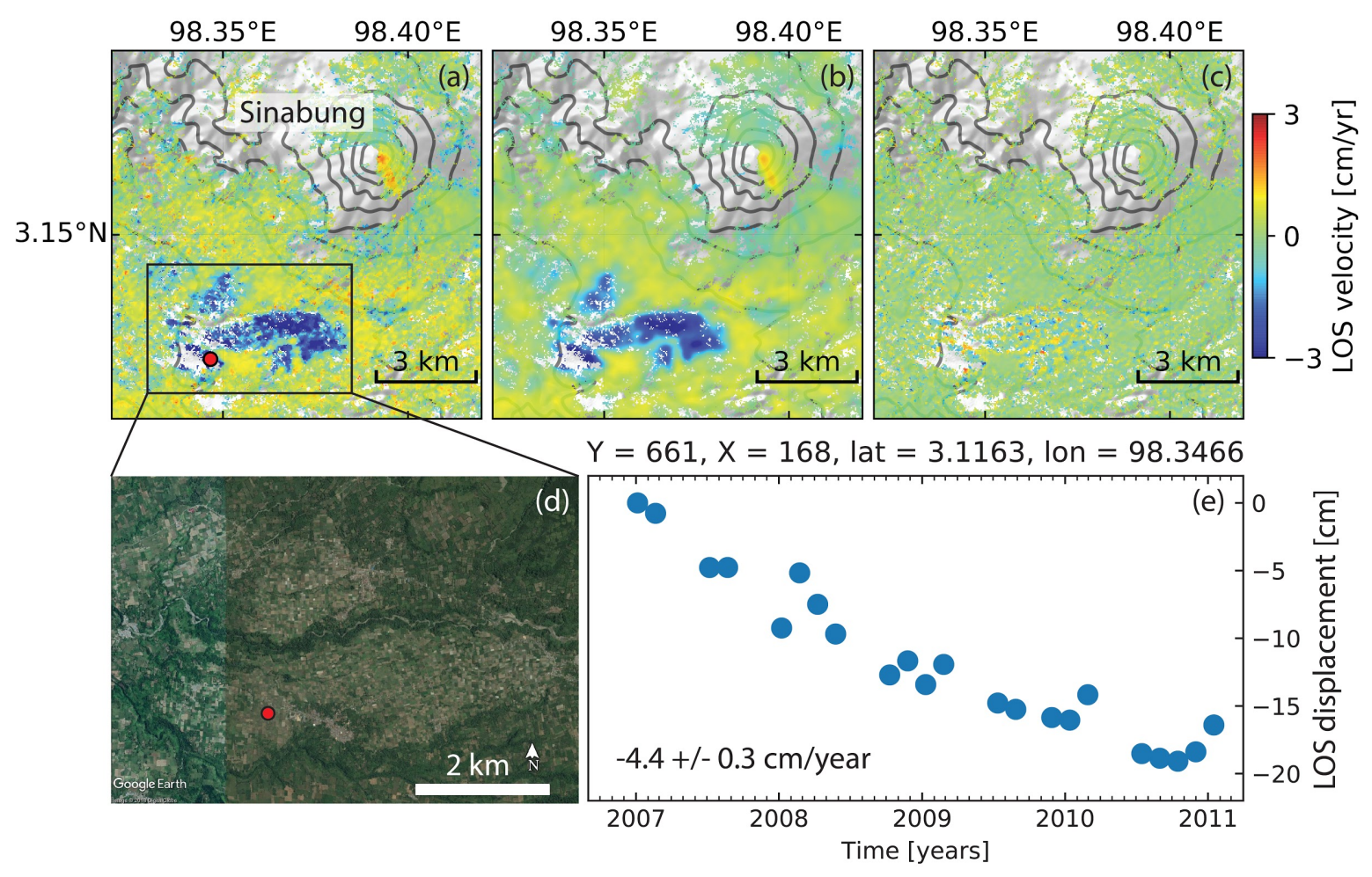

284 Figure S15. Illustration of the spatial filtering. The LOS velocity from ALOS-1 ascending track 285495 acquired over Sinabung volcano, Indonesia during January 2007 to January 2011 is used.

286 (a) Original velocity in LOS direction, (b and c) velocities after lowpass and highpass Gaussian 287 filtering with the standard deviation of 3.0. (a) is the sum of (b) and (c). The lowpass filtering 288 eliminated the very short spatial wavelength features, thus, highlighted the relatively long spatial 289 wavelength deformation features, such as the volcanic deformation along the Sinabung's 290 southeast flank and an undocumented patchy, rapid subsidence area (up to -5.6 cm/year) is 291 found $\sim 6 \mathrm{~km}$ to the southwest of the volcano. The spatial pattern of the subsidence signal 292 correlates well with the agricultural land use, suggesting that subsidence is caused by 293 groundwater extraction (Chaussard et al., 2013). Reference point is a pixel at [E98.4999, $294 N 3.1069^{\circ} \mathrm{J}$ outside of this figure. (d) Google Earth image for the marked rectangle area. (e) LOS 295 displacement time-series for pixel marked by red circle in (a) at [E98.3466,$\left.N 3.1163^{\circ}\right]$. 


\section{S4.3 Interferometric pairs selection}

297 The software supports several interferometric pairs selection methods to facilitate the pre298 processing, such as small baseline, sequential, hierarchical, Delaunay triangulation, minimum 299 spanning tree and star/PS-like methods, as shown in Fig. S16.
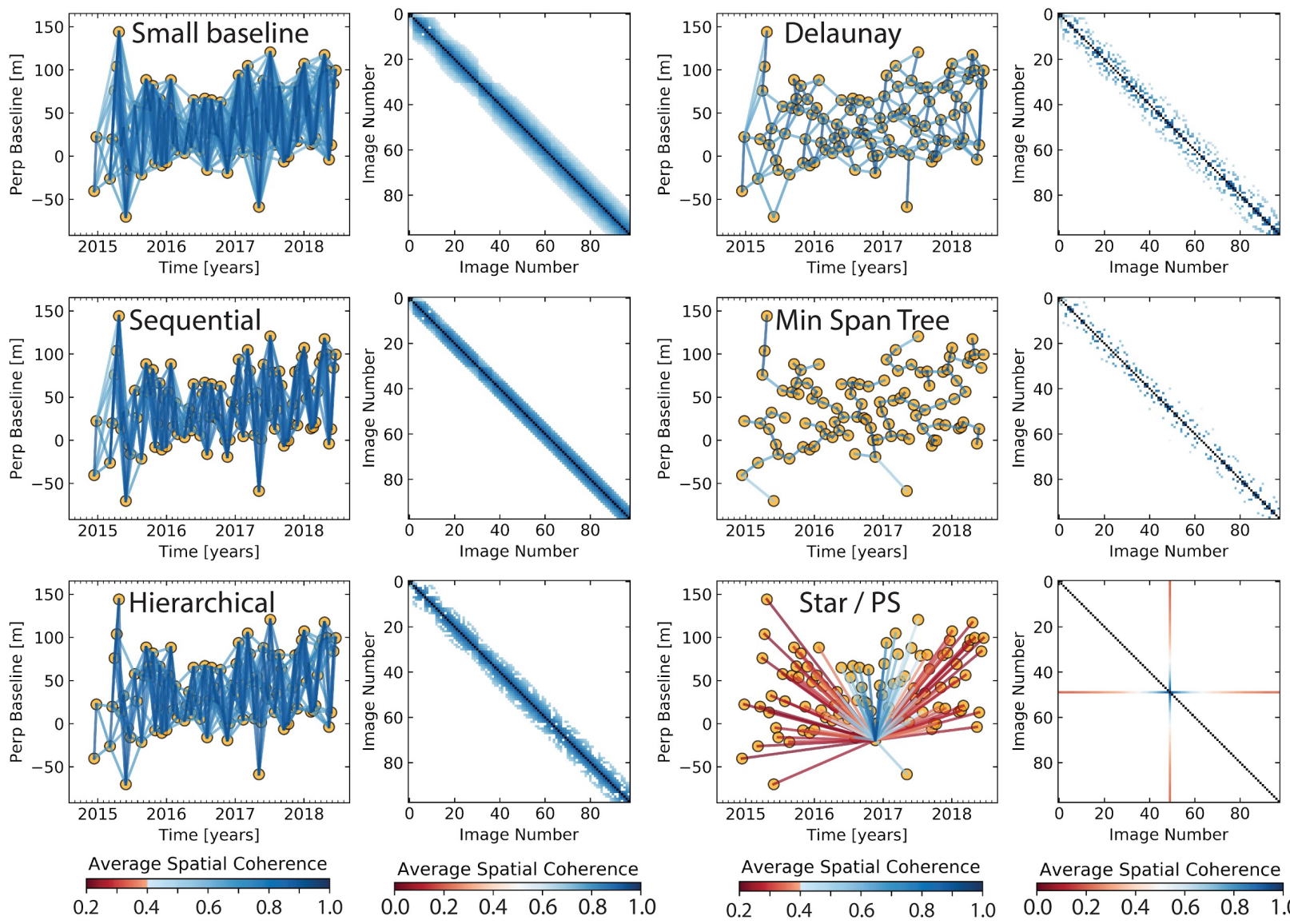

\begin{tabular}{c}
\multicolumn{5}{c}{ Average Spatial Coherence } \\
$\begin{array}{cccccc}0.0 & 0.2 & 0.4 & 0.6 & 0.8 & 1.0\end{array}$
\end{tabular}

301 Figure S16. Illustration of interferometric pairs selection. The temporal and perpendicular

302 baselines are from Sentinel-1 dataset of section 5. For each method, network configuration on

303 the left and the corresponding coherence matrix on the right. The spatial coherence calculation

304 is described in section S3.1 with decorrelation rate of 200 days and long-term coherence of 0.2.

305 The small baseline method selects interferograms with temporal and perpendicular baseline

306 within the predefined thresholds (120 days and $200 \mathrm{~m}$; Berardino et al., 2002). The sequential

307 method selects for each acquisition with a predefined number (5) of its nearest neighbors back in 
308 time (Reeves and Zhao, 1999). The hierarchical method specifies a predefined list of temporal 309 and perpendicular baselines as [6 days, $300 \mathrm{~m} ; 12$ days, $200 \mathrm{~m}$; 48 days, $100 \mathrm{~m}$; 96 days, $50 \mathrm{~m}$ ],

310 each pair of temporal and perpendicular thresholds selects interferograms the same as small

311 baseline method (Zhao, 2017). The Delaunay triangulation method generates triangulations in

312 the temporal and perpendicular baseline domain and selects interferograms within the

313 predefined maximum temporal and perpendicular baseline (120 days and $200 \mathrm{~m}$; Pepe and

314 Lanari, 2006). The minimum spanning tree method calculates a spatial coherence value based

315 on its simple relationship with the temporal and perpendicular baseline and selects $N-1$

316 interferograms that maximizes the total coherence (Perissin and Wang, 2012). The star-like

317 method selects network of $N-1$ interferograms with single common reference acquisition (usually

318 in the center of the time period; Ferretti et al., 2001).

\section{S4.4 Local oscillator drift correction for Envisat}

321 Data from Envisat's Advanced Synthetic Aperture Radar instrument include a phase ramp in 322 range direction due to timing errors. We correct this local oscillator drift using the empirical 323 model given by Marinkovic and Larsen (2013).

$$
\phi_{L O D}^{i}=\frac{-4 \pi}{\lambda} 3.87 \times 10^{-7} r\left(t_{i}-t_{1}\right)
$$

327 where $\left(t_{i}-t_{1}\right)$ represents the time difference in years between SAR acquisition $t_{i}$ and $t_{1}$ (see

328 also Fattahi and Amelung, 2014). Since this model is independent of the InSAR phase

329 measurement, this correction should be applied before any InSAR data-dependent phase 330 corrections. 


\section{Supplemental references}

332 Chaussard, E., F. Amelung, H. Abidin, and S.-H. Hong (2013), Sinking cities in Indonesia:

333 ALOS PALSAR detects rapid subsidence due to groundwater and gas extraction, Remote 334 Sensing of Environment, 128(0), 150-161, doi:10.1016/j.rse.2012.10.015.

335 Du, Y., L. Zhang, G. Feng, Z. Lu, and Q. Sun (2017), On the Accuracy of Topographic 336 Residuals Retrieved by MTInSAR, IEEE Transactions on Geoscience and Remote Sensing, 337 55(2), 1053-1065, doi:10.1109/TGRS.2016.2618942.

338 Marinkovic, P., and Y. Larsen (2013), Consequences of long-term ASAR local oscillator 339 frequency decay - An empirical study of 10 years of data, paper presented at Proceedings of 340 the Living Planet Symposium (abstract), European Space Agency, Edinburgh, U. K.

341 Reeves, S. J., and Z. Zhao (1999), Sequential algorithms for observation selection, IEEE 342 Transactions on Signal Processing, 47(1), 123-132, doi:10.1109/78.738245.

343 van der Walt, S., J. L. Schönberger, J. Nunez-Iglesias, F. Boulogne, J. D. Warner, N. Yager, E.

344 Gouillart, and T. Yu (2014), scikit-image: image processing in Python, PeerJ, 2, e453, 345 doi: $10.7717 /$ peerj.453.

346 Zhao, W. (2017), Small Deformation Detected from InSAR Time-Series and Their Applications 347 in Geophysics, Dissertation thesis, 153 pp, University of Miami, Miami, FL. 